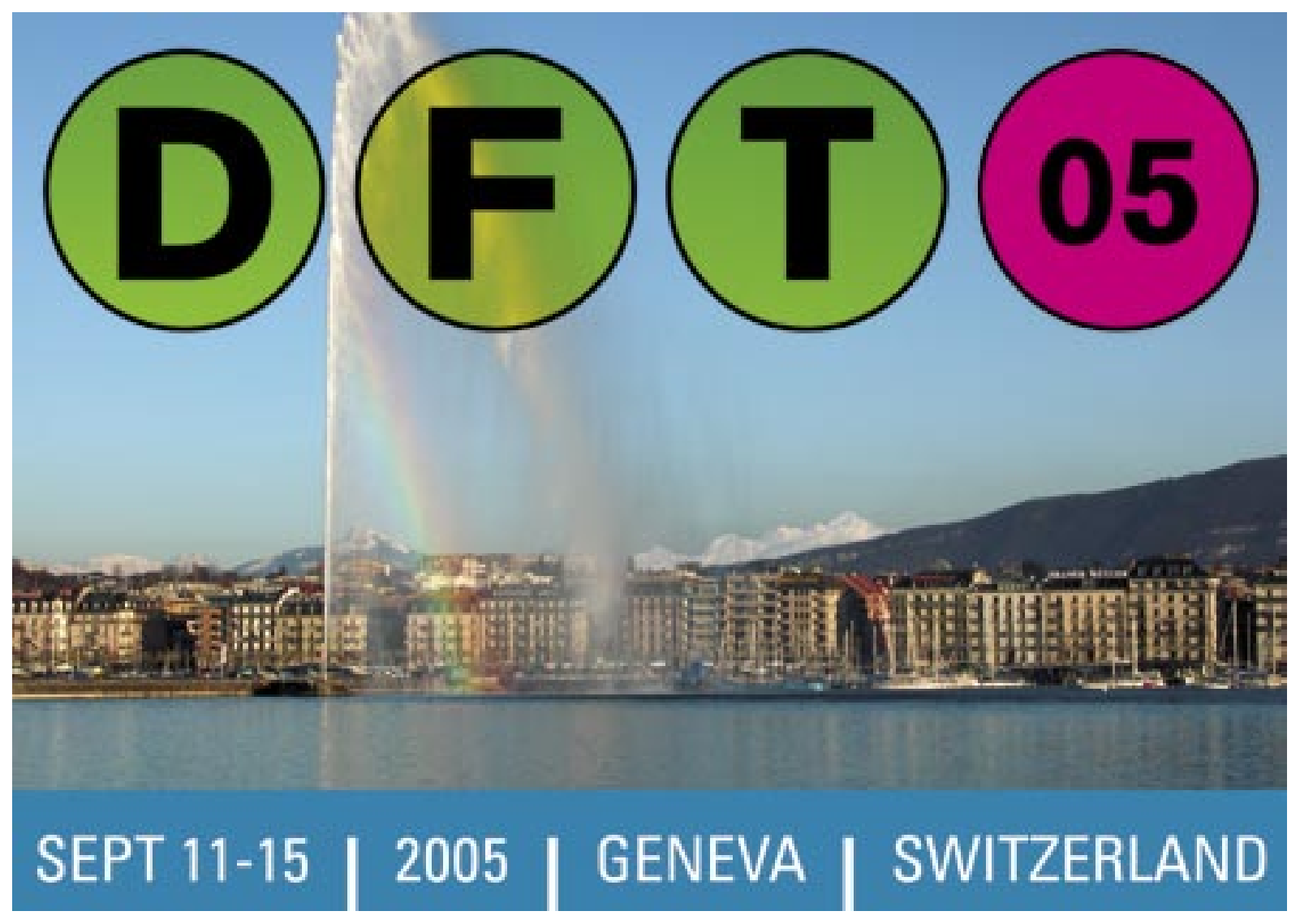

\title{
ABSTRACTS OF POSTERS
}

Session I: P1-P137

Session II: P201-P337 
Methodology and Applications

\section{DENSITY SCALING AND EXCHANGE-CORRELATION ENERGY}

\section{Á Nagy}

Department of Theoretical Physics, University of Debrecen, and Atomic and Molecular Physics Research Group of the Hungarian Academy of Sciences, H-4010 Debrecen, Hungary

A novel way of determining exchange-correlation energy is presented based on the density scaling proposed by Chan and Handy [1]. It is shown that there exist a value of the scaling factor for which the correlation energy disappears. The OPM and KLI methods are generalized to incorporate correlation. The $\zeta$ KLI method as simple as the original KLI method is proposed.

[1] G. K.-L. Chan and N. C. Handy, Phys. Rev. A, 1990, 59 , 2670 .
Methodology and Applications

P2

An improved density matrix functional by physically motivated orbitaldependent repulsive corrections

Oleg Gritsenko (1), Katarzyna Pernal (1,2), and Evert Jan Baerends(1)

(1) Afdeling Theoretische Chemie, Vrije Universiteit, De Boelelaan1083, 1081 HV Amsterdam, The Netherlands

(2) Institute of Physics, University of Szczecin, Wielkopolska 15, 70-451, Szczecin, Poland

Functionals of density matrix functional theory (DMFT) can serve as a paradigm of (virtual) orbital-dependent functionals of DFT. To fulfil this promise, however, the quality of the existing DMFT energy functionals should be considerably enhanced. This problem is tackled by developing an improved density matrix functional (BBC), in which a hierarchy of physically motivated corrections is employed to the strongly overbinding functional of Buijse and Baerends (BB, also known as the corrected Hartree, $\mathrm{CH}$, functional). The leading correction $\mathrm{C} 1$ restores the repulsive exchangecorrelation (xc) interaction between electrons in weakly occupied natural orbitals (NOs) as it appears in the exact electron pair-density for the limiting two-electron case. The second correction $\mathrm{C} 2$ reduces the $\mathrm{xc}$ interaction of the BB functional between electrons in strongly occupied NOs to an exchangetype correction. A similar correction $\mathrm{C} 3$ is also applied for the interaction of the antibonding orbital of a dissociating molecular bond. With these corrections, BBC still retains a correct description of strong non-dynamical correlation for the dissociating electron pair bond. BBC greatly improves the quality of the $\mathrm{BB}$ potential energy curves for the prototype few-electron molecules and in several cases BBC reproduces very well the benchmark $a b$ initio potential curves. The average error of the self-consistent correlation energies obtained with BBC3 for prototype atomic and molecular systems is only ca. $6 \%$.
Methodology and Applications

NEGF-DFT approach to photochemical reactions on surfaces

\section{Hisao Nakamura}

Department of Chemical System Engineering, Graduate School of Engineering, University of Tokyo, Tokyo 113-8656, Japan

An elementary process of substrate mediated photoreaction on surfaces contains the following steps: (1) electron-hole pair creation in the substrate, (2) transport to the surface region with relaxation, (3) attachment of hot electrons (holes) to adsorbate by tunneling, (4) promotion of nuclear dynamics (chemical reaction).

In this presentation, we propose a theoretical model based on non equilibrium Green's function (NEGF) theory to apply the above interfacial electron dynamics. The developed theory contains "semi-infiniteness" of the substrate without any arbitrary truncation, and gives the expression of photon energy dependence of a cumulative reaction probability:

$$
\begin{aligned}
& P_{\text {cum }}(E, \omega)=\frac{2}{\pi} \operatorname{Tr}\left[\operatorname{Im}\left(\Sigma_{e N}\right) G_{C}^{r} \operatorname{Im}\left(\Sigma_{\text {sub }}\right) G_{C}^{a}\right] f(E, \omega) \theta_{h}\left(E-E_{F}\right) \\
& f(E, \omega)=\frac{-\pi}{\Delta T} \frac{N(E, \omega)}{\operatorname{Tr}\left[\operatorname{Im} g_{\text {sur }}(E)\right]}
\end{aligned}
$$

For a practical calculation, we treat an optical response of the substrate semiclassically, and map to the quantum mechanical self-energy term. We combined the theory with DFT then applied it to the photoinduced reactions of $\mathrm{O}_{2}$ on $\mathrm{Ag}(110)$ surface and $\mathrm{NO}$ on $\mathrm{Ag}(111)$ surface.
Methodology and Applications

An Information Theory approach for the Construction of a Similarity Index for Atoms

\section{A. Borgoo, F. De Proft, P. Geerlings}

Eenheid Algemene Chemie (ALGC) Vrije Universiteit Brussel, Pleinlaan 2, 1050 Brussel, Belgium

In our work we investigate Quantum Similarity for atoms. Our goal is to construct a Similarity index which reveals the periodicity of the physical and chemical properties, which are conceiled in the densities and shape functions throughout the Mendeleev Table. Earlier work showed that the mear application of the Carbo Similarity Index ${ }^{1}$, used for comparing molecular systems, does not show the sought characteristics. Inspired by some recent publications we introduced the concept of Information Entropy. Evaluating this information theory based functional for each atom, with its core as reference did uncover a periodic evolution through Mendeleev's Table, with improved results when the shape function is used to evaluate the Entropy functional ${ }^{2}$. This confirms recent literature on the fundamental role of the shape function as carrier of information ${ }^{3}$. We are currently devising a similarity index, based on the entropy functional, aiming for a periodic similarity index.

[1] R. Carbó-Dorca, , J. Arnau, L. Leyda, Int. J. Quant. Chem., 1980, 17, 1185.

[2] A. Borgoo, M. Godefroid, K. D. Sen, F. De Proft, P. Geerlings, Chem. Phys. Let., 2004, 399, 363

[3] P. W. A. Ayers, Proc. Natl. Acad. Sci., 1995, 17, 2000 
Methodology and Applications

Role of Local and Global DFT based indices in the Estimation of Interaction Energy Components of Stacked Aromatic Molecules

\author{
Pierre Mignon, Stefan Loverix, Paul Geerlings.
}

Eenheid Algemene Chemie (ALGC)

Faculteit Wetenschappen, Vrije Universiteit Brussel,

Pleinlaan 2, 1050 Brussels, Belgium.

A set of stacked complexes comprising aromatic nitrogen bases/nucleobases and substituted benzenes has been studied to test local and global DFT based reactivity indices ${ }^{1}$ for their ability to predict interaction energy components. From its relationship with the softness and polarisability, the global hardness index has been tested to estimate the correlation interaction energy (dispersion energy) between pyridine stacked in parallel displaced arrangements with substituted benzenes ${ }^{2}$. Likewise, the benzene ring polarisability was successfully used to predict the dispersion interaction between various aromatic nitrogen bases and nucleobases stacked with substituted benzenes ${ }^{3}$. Finally the local hardness computed above the benzenes' rings or in para position from the substituent, performed nicely to predict the electrostatic interaction, for parallel as well as T-shaped arrangements. Moreover the local hardness appeared to be a key index relating the electrostatic interaction between benzenes and the aromatic base and the hydrogen bonding ability of the latter ${ }^{4}$. This presentation includes also a brief presentation of the effect of the stacking interaction on the hydrogen bonding ability of aromatic bases and nucleobases.

[1] Geerlings, P.; De Proft, F.; Langenaeker, W. Chem. Rev. 2003, 103 , 1793.

[2] Mignon, P.; Loverix, S.; De Proft, F.; Geerlings, P. J. Phys. Chem. A 2004, 108, 6038.

[3] Mignon, P.; Loverix, S.; Geerlings, P. Chem. Phys. Lett. 2005, 401, 40.

[4] Mignon, P.; Loverix, S.; Steyaert, J.; Geerlings, P. Nucleic Acids Res. 2005, Accepted.

\section{Methodology and Applications}

Assessment of the performance of the Perdew-Zunger self-interaction corrected density functional theory

Oleg A. Vydrov and Gustavo E. Scuseria

Department of Chemistry, Rice University,

6100 Main Street, Houston, Texas 77005

The Perdew-Zunger self-interaction-corrected (PZ-SIC) density functional theory was implemented self-consistently using a quasi-Newton direct minimization method [1]. Our implementation works with Gaussian basis sets and can be used with a variety of exchange-correlation functionals including hybrids. We calculated enthalpies of formation of the standard G2-1 test set and found that PZ-SIC improves agreement with experiment only for the LSDA functional, while all other functionals show worse performance upon introducing PZ-SIC [1].

We also calculated ionization potentials (IP) and electron affinities (EA) of first- and second-row atoms and a set of small molecules [2]. IPs and EAs were obtained by two methods: as the difference in SCF energies of neutrals and ions $(\triangle \mathrm{SCF})$, and as negatives of highest occupied orbital energies. We found that, except for LSDA, PZ-SIC worsens $\triangle$ SCF IPs and EAs. On the other hand, PZ-SIC brings orbital eigenvalues into much better agreement with electron removal energies. Spherical averaging of orbital densities, which is commonly used in approximate PZ-SIC calculations in atoms, was found to improve the performance of PZ-SIC for GGA functionals.

We also demonstrated that PZ-SIC improves description of odd-electron systems and prediction of activation barriers of chemical reactions. Overall, the performance of PZ-SIC is ambivalent. PZ-SIC tends to overcorrect many-electron systems and in some cases it impairs accuracy of approximate functionals.

[1] O. A. Vydrov and G. E. Scuseria, J. Chem. Phys., 2004, 121, 8187.

[2] O. A. Vydrov and G. E. Scuseria, to appear in J. Chem. Phys.

Methodology and Applications

P6

The Nuclear Fukui Function in the Context of the Spin Polarized Density Functional Theory.

\author{
Eduardo Chamorro $^{1}$, Frank De Proft ${ }^{2}$, Paul Geerlings ${ }^{2}$
}

Department of Chemistry, Universidad Andrés Bello. Av. República 275, Santiago. Chile. ${ }^{1}$ Department of Chemistry. Vrije Universiteit Brussel Pleinlaan 2 - 1050 - Brussel $^{2}$

Density functional theory (DFT) has provided a fruitful framework for the development of a powerful theory of chemical reactivity. In this framework, electronic and/or nuclear reactivities are naturally described in terms of the coefficients of Taylor expansions for the electronic and/or total energy functionals. Complete hierarchies of chemical descriptors are understood in terms of global and local responses of the system to global or local perturbations.

In this work, we focus on the definition, and application of a nuclear Fukui function within the context of the spin polarized DFT, ${ }^{2}$ which measures the magnitude and direction for the change of the force $\mathbf{F}_{\alpha}$ acting on nucleus $\alpha$, under changes in the spin number Ns, at fixed external potential $\mathrm{v}(\mathbf{r})$ and total number of electrons $\mathrm{N}$,

$$
\Phi_{\alpha, s}=\left[\frac{\partial \mathbf{F}_{\alpha}}{\partial N_{s}}\right]_{N, v(\mathbf{r})} .
$$

Such a quantity will provide new insights about molecular geometry changes upon excitation. In this work, we explore the derivation and the relationship of this descriptor with the other electronic and nuclear indexes in a canonical ensemble [N,Ns, $\mathrm{v}(\mathbf{r})]$, as well as its connection with chemical binding using a spin analog of Berlin's function.

[1] Geerlings P.; De Proft F.; Langenaeker W. Chem. Rev. 2003, 103, 1793.

[2] Galvan, M.; Vela, A; Gazquez J. L. J. Phys. Chem. 1988, 92, 6470; Galvan, M. Vargas, R. J. Phys. Chem. A. 1992, 96, 1625.

Methodology and Applications

Open-Shell Localized Hartree-Fock Method For a Self-Consistent Treatment of Excited States

$\underline{\text { Fabio Della Sala }^{1}}$, Vincenzo Vitale $^{1}$, and Andreas Görling ${ }^{2}$

${ }^{1}$ National Nanotechnology Laboratory of INFM

Università degli Studi di Lecce, Lecce, Italy

${ }^{2}$ Lehrstuhl für Theoretische Chemie

Universität Erlangen-Nürnberg, Erlangen, Germany

The Open-Shell Localized Hartree-Fock (OSLHF) method [1] is extended within the Generalized Adiabatic Connection Kohn-Sham formalism to treat excited electronic states which are not the energetically lowest of their symmetry. The method is numerically stable, self-interaction free and allows for a fully self-consistent computation of excited valence as well as Rydberg states of molecules. In contrast to the common timedependent density-functional methods, the OSLHF approach is capable of treating doubly or multiply excited states and can be applied to molecules with an open-shell ground state and charge-transfer systems.

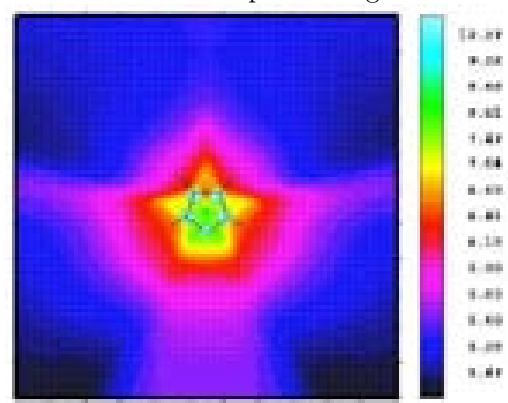

Fig.1 'Correction term' of the OSLHF potential for the $3{ }^{1} A_{1}$ state of Furan.

[1] F. Della Sala and A. Görling, J. Chem. Phys., 2003, 118, 10439.

[2] F. Della Sala and A. Görling, Phys. Rev. Lett., 2002, 89, 033003.
The local multiplicative Kohn-Sham exchange potential for a given singlet, triplet or multiplet excitedstate can be obtained. We show that on nodal surfaces of the energetically highest occupied orbital the OSLHF exchange potential asymptotically approaches a different, i.e., nonzero, value than in other regions [2]. 
Methodology and Applications

Time-Dependent Density-Functional Theory study of singlet and triplet excitation energies in oligothiophenes

Eduardo Fabiano ${ }^{1}$, Fabio Della Sala ${ }^{1}$, Roberto Cingolani ${ }^{1}$, Martin Weimer $^{2}$ and Andreas Görling ${ }^{2}$

${ }^{1}$ National Nanotechnology Laboratory of INFM

Università degli Studi di Lecce, Lecce, Italy

${ }^{2}$ Lehrstuhl für Theoretische Chemie

Universität Erlangen-Nürnberg, Erlangen, Germany

The theoretical investigation of the chain-length evolution of the relative energetic position of the singlet and triplet excited states in oligothiophenes is of fundamental importance to understand the photophysics of these materials. This issue has so far been investigated either by semiempirical methods [1] or by ab-initio calculations for small oligomers only $[2]$.

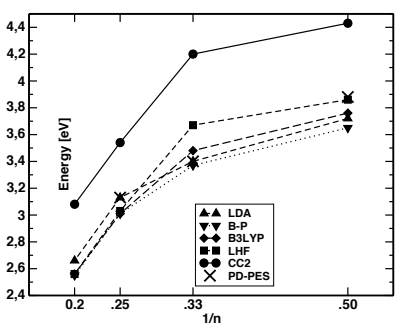

In this work explore the ability, still under debate [3], of time-dependent density-functional theory (TD-DFT) to correctly describe the relative energetic position of singlet and triplet states in oligothiophenes. We study excitation energies using TD-DFT with different exchange-correlation functionals and compare the results with ab-initio results and experimental data [4]

We find that TD-DFT can give a good description of the chain length evolution, especially with an hybrid kernel, and it is used for the reassigment of experimental data [5].

[1] D. Beljionne, et al., J. Am. Chem. Soc., 1996, Vol, 118, 6453.

[2] M. Rubio, et al., Chem. Phys. Chem., 2003, 4, 1308.

[3] S. Grimme, and M. Parac, Chem. Phys. Chem., 2003, 4, 292.

[4] S. Rentsch et al., Phys. Chem. Chem. Phys. 1999, 1, 1707.

[5] E. Fabiano et al., J. Phys. Chem A. 2005 in press.

Methodology and Applications

Applications of long-range-corrected density functional theory

\section{Takao Tsuneda}

Department of Quantum Engineering and Systems Science, Graduate School of Engineering, The University of Tokyo, Tokyo 113-8656, Japan

A long-range-corrected (LC) density functional theory (DFT) [1] was applied to calculations of various systems, which have been poorly evaluated by conventional DFTs: van der Waals (vdW) complexes [2,3], charge transfer (CT) complexes [4], nonlinear optical systems [5], reaction systems, and biological systems. For vdW complexes, we calculated potential energy surfaces of rare-gas dimers [2,3], diatomic molecules with He complexes [3], and benzene dimers by combining the LC exchangecorrelation functional with Andersson-Langreth-Lundqvist (ALL) vdW functional. As a result, we found that this LC-DFT+ALL functional gave very accurate potential energy surfaces even with small basis sets equivalent of the second-order Møller-Plesset perturbation results calculated with very large basis sets. We also calculated electronic excitation energies including CT energies by applying the LC scheme to the time-dependent DFT (TDDFT). Consequently, we confirmed that the LC scheme obviously improved excitation energies of various systems especially for CT energies of ethylene-tetrafluoroethylene dimer [4] and 4N,N-dimethylamino-benzonitrile (DMABN). Following these results, the LC method was applied to excited-state molecular dynamics based on TDDFT. We first ran the simulation of the cis-trans isomerization of protonated retinal, which is known to proceed through the intramolecular CT in $\pi$-orbitals of retinal. As a result, we succeeded in tracing whole isomerization process within the framework of TDDFT. Besides, we found that overestimated optical properties and reaction energy barriers were both fairly improved by the LC scheme.

[1] H. Iikura, T. Tsuneda, T. Yanai, and K. Hirao, J. Chem. Phys., 2001, 115, 3540. [2] M. Kamiya, T. Tsuneda and K. Hirao, J. Chem. Phys., 2002, 117, 6010 [3] T. Sato, T. Tsuneda and K. Hirao, Mol. Phys., in press.

[4] Y. Tawada, T. Tsuneda, S. Yanagisawa, T. Yanai and K. Hirao, J. Chem. Phys. 2004, 120, 8425.

[5] M. Kamiya, H. Sekino, T. Tsuneda, and K. Hirao, J. Chem. Phys., in press.
Extended constrained-search theory: Arbitrary choice of basic variables in density functional theory

\section{Masahiko Higuchi ${ }^{1}$ and Katsuhiko Higuchi ${ }^{2}$}

Department of Physics, Faculty of Science ${ }^{1}$

Shinshu University, Matsumoto 390-8621, Japan

Graduate School of Advanced Sciences of Matter ${ }^{2}$

Hiroshima University, Higashi-Hiroshima 739-8530, Japan

We have recently proposed the extended constrained-search (ECS) theory in which arbitrary physical quantities can be chosen as basic variables in addition to the electron density. ${ }^{1-3}$ The ECS theory has two advantages over the conventional density functional theory (DFT). One is that the ground state values of quantities which characterize the system can be reproduced directly by means of the Kohn-Sham orbitals, simultaneously with the ground state value of the electron density. Another is that the approximate form of the exchange-correlation energy functional may be given in more efficient form than the conventional DFT.

The validity of this theory has been confirmed by revisiting the previous theories such as the spin-density functional theory, current-density functional theory, LDA $+U$ method and Hartree-Fock-Kohn-Sham scheme. For example, if the occupation matrix of localized orbitals is chosen as one of basic variables, then we can obtain the single-particle equation which is equivalent to that of the $\mathrm{LDA}+U$ method.

Furthermore, toward the actual energy-band calculation within the ECS scheme, we have derived the exact expression for the exchange-correlation energy functional by using the coupling-constant technique. It is expected to be a good starting point for developing the approximate form of the ECS exchange-correlation energy functional.

[1] M. Higuchi and K. Higuchi, Phys. Rev. B, 2004, 69, 035113.

[2] K. Higuchi and M. Higuchi, Phys. Rev. B, 2004, 69, 165118.

[3] K. Higuchi and M. Higuchi, Phys. Rev. B, 2005, 71, 035116.

Methodology and Applications

Improving the common-energy-denominator approximation to the exchange potential.

\author{
A. Holas and M. Cinal \\ Institute of Physical Chemistry of the Polish Academy of Sciences,
} 44/52 Kasprzaka, 01-224 Warsaw, Poland

It is shown that the accurate approximation to the exchange potential (XP) derived by Gritsenko and Baerends [1] (named the common-energydenominator approximation) and the equivalent one derived by Della Sala and Görling [2] (named the localized Hartree-Fock approximation) can be improved. For the spin subsystem described by $N$ occupied Kohn-Sham orbitals, we define an approximate XP (AXP) which depends on $(N-1)$ free parameters. By setting all parameters equal 1, our AXP reduces to the previous one. The parameters can be varied to optimize this AXP. It can be shown that the original AXP follows from the "optimized effective potential" equation [which defines the exact XP (EXP)] after replacing in it all energy denominators by a common value. The present AXP corresponds to replacing there selected groups of denominators by common values. The large- $r$ asymptotics $-1 / r$ of the EXP is reproduced by our AXP for any fixed set of parameters. Criteria of optimization of this AXP are discussed and results shown on examples.

[1] O.V. Gritsenko, E.J. Baerends, Phys. Rev. A, 2001, 64, 042506.

[2] F. Della Sala, A. Görling, J. Chem. Phys., 2001, 115, 5718. 
Methodology and Applications

\section{Exchange Representations in Kohn-Sham Theory}

Andrew M Teale and David J Tozer

Department of Chemistry, University of Durham, South Road, Durham, DH1 3LE, UK

Following our recent work[1,2] using LHF exchange-only theory[3], we extend our investigation to compare several finite basis set exchange-only Kohn-Sham methods. We perform calculations using the Slater[4], KLI[5], LHF, ZMP[6], OEP[7,8], WY(HF)[9], and LDAX[10] exchange representations. Specifically, NMR shielding constant calculations are used as a sensitive probe to highlight subtle differences in the exchangecorrelation potentials, densities, and frontier orbitals obtained from each approach. The importance of the HOMO-LUMO gap to the calculation of NMR shielding constants is reiterated. A strong spatial correlation between differences in the potentials and densities is observed and is reflected in a comparison of the molecular orbitals obtained from the various exchange only methods.

\section{References}

[1] A.M. Teale, D.J. Tozer, Chem. Phys. Lett., 2004, 383, 109.

[2] A.M. Teale, D.J. Tozer, J. Chem. Phys., 2005, 122, 034101.

[3] F. Della Sala, A. G"orling, J. Chem. Phys., 2001, 115, 5718 .

[4] J.C. Slater, Phys. Rev., 1951, 81, 385.

[5] J.B. Krieger, Y. Li, G.J.Iafrate, Phys. Rev. A,1992,45, 101.

[6] Q.Zhao, R.C.Morrison, R.G.Parr,Phys. Rev. A,1994,50,2138.

[7] J.D. Talman, W.F. Shadwick Phys. Rev. A, 1976, 14, 36.

[8] W. Yang, Q. Wu, Phys. Rev. Lett., 2002, 89, 143002.

[9] W. Yang, Q. Wu, J. Chem. Phys., 2003, 118, 2498.

[10] L.J. Sham, W. Kohn, Phys. Rev., 1965, 140, A1133.

Methodology and Applications

\section{Optimized Effective Potentials in Current Density Functional Theory}

\section{S. Pittalis, S. Kurth, N. Helbig and E.K.U. Gross}

Free UniversityBerlin, Arnimallee 14 D-14195 Berlin

The proper description of many-electron systems in the presence of magnetic fields within a density-functional framework requires the current density to be used as basic variable besides the electron density. Unlike in ordinary spin-density functional theory (SDFT), where only the coupling of the magnetic field to the spin degrees of freedom is taken into account, in current-density-functional theory (CDFT)one also allows for the coupling to the orbital currents. Moreover, in the limit of zero external magnetic fields the Kohn-Sham (KS) equations in CDFT do not reduce to the KS equations in SDFT. Electron-gas-based (LDA-type) functionals of CDFT exhibit derivative discontinuities as a function of the magnetic field whenever a new Landau level is occupied which makes them difficult to use in practice. However, since the appearance of Landau levels intrinsically is an orbital effect, it is appealing to use orbital-dependent functionals. Here, we present an optimized effective potential (OEP) method within a CDFT framework which allows for the use of explicitly orbital-dependent functionals in selfconsistent computations. The derivation of these equations and a numerically tractable scheme for their solution within a KLI-type approximation is presented. Numerical results at the exact exchange-only level for open shell atoms without external magnetic field, and quantum dots in an external magnetic field are discussed.
Methodology and Applications

P14

\section{Semi-empirical fitting of exchange-correlation functionals}

\author{
Thomas W Keal and David J Tozer
}

Department of Chemistry, University of Durham, South Road, Durham DH1 3LE, UK

We describe our continuing work to improve the quality of semi-empirical exchange-correlation functionals in DFT by fitting to a diverse range of properties with a large number of training molecules. This highly empirical approach can result in significant improvements in accuracy over competing generalised gradient approximation (GGA) and hybrid functionals.

Previous studies $[1,2]$ have shown that a simple gradient correction to the local density approximation can provide NMR shielding constants for challenging main-group nuclei that are 2-3 times more accurate than those of conventional GGAs and hybrids. These functionals were denoted KT1 and KT2. By adding one additional exchange term, the KT3 GGA functional was developed [3], which retained the quality of shielding constants while substantially improving the performance for other properties. In particular, it gives atomisation energies that are as accurate as the best competing GGA functionals and equilibrium molecular bond lengths that are as accurate as the best hybrid functionals. However, its performance for classical reaction barriers is relatively weak. We describe our attempts to develop new GGA and hybrid functionals that give improved reaction barriers while retaining the high quality performance of KT3 for other properties.

[1] T.W. Keal and D. J. Tozer, J. Chem. Phys. 2003, 119, 3015.

[2] M.J. Allen, T.W. Keal and D.J. Tozer, Chem. Phys. Lett. 2003, 380, 70.

[3] T.W. Keal and D.J. Tozer, J. Chem. Phys. 2004, 121, 5654.

Methodology and Applications

\section{A Practical Scheme for Quantum Transport using Time-Dependent} Density Functional Theory

$\underline{\text { S. Kurth }}{ }^{1}$, G. Stefanucci ${ }^{2}$, C.-O. Almbladh ${ }^{2}$, A. Rubio ${ }^{3}$, E.K.U. Gross ${ }^{1}$

Institute for Theoretical Physics, Free UniversityBerlin, Germany

2Solid State Theory, Lund University, Lund, Sweden

${ }^{3}$ Donostia International Physics Center, San Sebastian/Donostia, Spain

The Landauer formalism is a popular method to calculate the current of noninteracting electrons through a mesoscopic or nanoscopic system connected to two (or more) macroscopic electrodes in the steady-state. Here we present a time-dependent description of transport based on the time evolution of the non-interacting Schrödinger equation. We develop a numerical algorithm for the time propagation of extended states. For simple model systems, the scheme is used to compute the time-dependent current in response to an external dc or ac bias. As expected, for a dc bias the system evolves to the steady state predicted by the Landauer formula. Using the algorithm in the framework of time-dependent density functional theory allows for the description of transport of interacting electrons beyond the Landauer formalism. 
Methodology and Applications

Nuclear Fukui Function from Non-integral Electron Number Calculations

Carlos Cárdenas", Eduardo Chamorro ", Marcelo Galvan and Patricio Fuentealba

a) Universidad Andrés Bello, Av. Républica 275, Santiago, Chile.

b) Universidad de Chile, Departamento de Física, Facultad de Ciencias, Casilla 653, Santiago, Chile.

c) Universidad Autónoma Metropolitana-Iztapalapa, Departamento de Química, Mexico DF, Mexico.

The nuclear Fukui function (NNF) introduced by Cohen et al. [1] has generated many interest in nuclear chemical reactivity context. It allows to describe the change of the forces on the nuclei associated to a perturbation in the number of electrons

$$
\Phi_{\alpha}=\left(\frac{\partial \mathrm{F}_{\alpha}}{\partial \mathrm{N}}\right)_{\mathrm{v}(\mathrm{r})}
$$

To date few works have been reported about this index, which are limited to diatomic molecules and the prediction of the Jahn-Teller distortion for a series of molecules [2]. Three schemes have been reported to calculate the NFF, namely: the finite difference approximation for energy gradient, the numerical interpolation of the chemical potential gradient and via closed-shell coupled perturbed HF equations [2]. In this work we report a new approximation to calculate the NFF: a numerical interpolation of the forces for non-integral occupation numbers based on the Janak version of the density functional theory [3].

[1] M. H. Cohen, M. V. Ganduglia-Pirovano, and J. Kudrnovsky, J. Chem. Phys. 1994, 101, 8988.

[2] a) Frank De Proft, Shubin Liu, and Paul Geerlings, J. Chem. Phys. 1998, 108, 7549. b) R. Balawender and Paul Geerling, J. Chem. Phys. 2001, 114, 682

[3] J. F. Janak, Phys. Rev. B. 1978, 18, 7165 .

Methodology and Applications

\section{Dispersion corrected atom centered potentials for density functional theory}

\section{O.Anatole von Lilienfeld ${ }^{\dagger}$, Enrico Tapavicza ${ }^{\dagger}$, I-Chun Lin ${ }^{\dagger}$, Maurício D. Coutinho-Neto $^{\dagger}$, Daniel Sebastiani ${ }^{\ddagger}$, Ivano Tavernelli ${ }^{\dagger}$, Ursula Rothlisberger $^{\dagger}$}

$\dagger$ Ecole Polytechnique Fédérale de Lausanne (EPFL), Laboratoire de Chimie et Biochimie Computationelle, 1015 Lausanne, Switzerland

$\ddagger$ Max-Planck-Institute for Polymer Research, Ackermannweg 10, 55128 Mainz, Germany

Recently, we have introduced a pragmatic scheme for the variational optimization of atom based nonlocal external potentials within the framework of density functional theory (DFT) in order to systematically improve the description of molecular properties [1]. Results for applying this approach to describe London dispersion forces within conventional generalized gradient approximation DFT calculations are presented [2-4]. Specifically, a small library of dispersion corrected atom centered potentials (DCACP) is obtained by calibration of typical van der Waals systems such as $\mathrm{Ar}_{2}, \mathrm{Kr}_{2},\left(\mathrm{Br}_{2}\right)_{2}$, and the benzene-dimer in its sandwich configuration. Then, the performance of these DCACPs is assessed for various weakly bonded complexes. We investigate (i) ideal van der Waals clusters of the type $\operatorname{Ar}_{n} \mathrm{Kr}_{m}(\forall n, m \in\{0,1,2,3,4\}$ and $2 \leq n+m \leq 4$ ), (ii) other configurations of the benzene dimer, (iii) the benzene and the graphite crystal, (iv) the hybridization for methane, ethane, ethylene, and acetylene dimers, (v) the effect of other predominant interactions (covalent bonds, hydrogen bonds, permanent dipole moments) for the cyclooctatetraene molecule and the hydrogen bromide dimer. While the computational cost remains constant for all the DCACP-DFT calculations, the transferability of the DCACPs to this extended set of complexes is remarkably good.

[1] O.A. von Lilienfeld, et al., J. Chem. Phys., 2005, 122, 14113

[2] O.A. von Lilienfeld, et al., Phys. Rev. Lett, 2004, 93, 153004.

[3] O.A. von Lilienfeld, et al., Phys. Rev. B, 2005, accepted.

[4] E. Tapavicza, et al., 2005, submitted.
Methodology and Applications

Empirical Energy-Density Relationships Applied to the Analysis of the Basicity of Strong Organic Superbases

E. Chamorro, C. A. Escobar, R. Sienra M. and P. Pérez

Departamento de Ciencias Químicas, Facultad de Ecología y Recursos Naturales, Universidad Andrés Bello. Av. República 275, Santiago. Chile.

The search and the rational design of strong organic superbases has received a great deal of experimental and theoretical attention during the last decades because their impact in medicinal, pharmaceutical, and organic synthesis applications.

In this work, the site selectivity and reactivity in some strong organic super bases containing the imino group, $\mathrm{RN}=\mathrm{C}\left(\mathrm{NR}_{2}\right) \mathrm{R}$ three or more possible protonation sites, have been analyzed qualitatively in terms of global and local chemical reactivity descriptors within the context of density functional theory. It has been found that the maximum regional Fukui function value correctly predicts the protonation site at the imino nitrogen in each superbase. Hammet-like energy-density linear relationships between global energy-dependent quantities (i.e. the proton affinity, PA) and the variation of the Fukui function $\Delta \mathrm{f}(\mathbf{r})$, or the local softness, $\Delta \mathrm{s}(\mathbf{r})$ at the imino nitrogen have been found

$$
\ln \left[\frac{P A}{P A^{0}}\right]=\gamma_{f} \Delta f_{k}^{-} \text {, or } \ln \left[\frac{P A}{P A^{0}}\right]=\gamma_{s} \Delta s_{k}^{-}
$$

It seems that these linear relationships could be interpreted within a local HSAB rule framework, in analogy with the gas-phase basicity of alkylamines and gas-phase acidity of alcohols and thioalcohols. ${ }^{2}$

Acknowledgements: This work was supported by Fondecyt, grants Nos. 1030173, an 1020069. We also thanks to UNAB, grants $08-03,16-04,15-04,41-04$ and 17-04. We are grateful to the Millennium Nucleus, grant $\mathrm{N}^{\circ} \mathrm{P} 02-004-\mathrm{F}$ (Mideplan and Conicyt).

[1] See for instance: Kovacevic, B.; Baric, D.; Maksic, Z.B. New J. Chem. 2004, 28, 284; Vianello, R.; Kovacevic, B.; Maksic, Z. B.; New J. Chem. 2002, 26, 1324.

[2] Pérez, P.; Simon-Manso, Y.; Aizman, A.; Fuentealba, P.; Contreras, R. Journal of the American Chemical Society 2000, 122, 4756.

Methodology and Applications

van der Waals interaction within DFT: From isolated polymer chains to crystal structure

J. Kleis $^{1}$. E. Schröder ${ }^{1}$, D. C. Langreth ${ }^{2}$, S. D. Chakarova ${ }^{1}$. P. Hyldgaand ${ }^{1}$, and B. I. Lundqvist ${ }^{1}$

Department of Applied Physics ${ }^{1}$, Chalmers Universily of Technology SE-41296 Göleborg, Sweden

Cenler for Malerials Theory ${ }^{2}$, Departmenl of Physics and Astronomy,

Rulgers Unicersily, Piscalaway, New Jersey 08854-8019, USA

Traditional local and semi-local implementations of density functional theory predict the intramolecular properties of polyethylene ( $\mathrm{PE}$ ) in excellent agreement with experiments. However, they do not include the non-local attractive van der Waals (vdW) forces responsible for stabilizing the polymer crystal structure.

As an attempt to remedy this deficit we have used two different approaches. For pairs of polymers separated beyond the binding distance (polymer separations $>\varepsilon-10 \AA$ ), we propose a systematic correction scheme within the framework of traditional time-independent DFT. In this approach we model the time-dependent response of the isolated polymers on the basis of their static polarization response and electron density. This allows us to calculate the polymer-polymer vdW interaction beyond the binding distance for a number of simple polymers [1].

For pairs of polyethylene and the PE crystal we use a recent scheme for calculating the vdW interaction in dimers [2] to correct for the non-local vdW interaction also at the binding distance on the basis of the groundstate electron density only. This approach yields cohesive energies and equilibrium lattice parameters of the PE-crystal in promising agreement with experiments.

At polymer separations beyond the binding distance the two approaches give results consistent with each other.

[1] J. Kleis and E. Schröder, Van der Waals interaction of simple, parallel polymers. To appear in J. Chem. Phys.

[2] M. Dion el al., Phys. Rev. Lett. 92 (2004) 246401. 
Methodology and Applications

\section{Stationarity principle for non-equilibrium states}

Matthias Ernzerhof and Sergey N. Maximoff

Department of Chemistry

University of Montreal

C.P. 6128 , Succursale A

Montreal, Quebec H3C 3J7

Canada

Stationary current transport through molecular electronic devices (MEDs) represents a non-equilibrium problem. We develop a pseudo-Hermitian Hamiltonian that yields eigenfunctions describing the non-equilibrium state. A stationarity principle for the pseudo-Hermitian Hamiltonian is constructed that resembles the variational method of conventional Hermitian quantum mechanics. This stationarity principle permits the use of many techniques of conventional electronic structure theory, such as the configuration interaction method. As an application of the stationarity principle, we develop a generalization of Kohn-Sham density functional theory suitable for MEDs.
Methodology and Applications

P22

Application of density functional theory to problems of molecular electronics

Matthias Ernzerhof, Min Zhuang, Sergey N. Maximoff, Philippe Rocheleau, and Hilke Bahmann

Department of Chemistry

University of Montreal

C.P. 6128, Succursale A

Montreal, Quebec H3C 3J7

Canada

Typically, a molecular electronic device (MED) consists of a molecule attached to two metal contacts. A key property of MEDs is their electrical conductance. The basic concepts governing current transport through molecules will be explained. We present different methods for the calculation of the conductance of MEDs that are derived from density functional theory. Various applications of our theoretical tools are discussed that aim at understanding the mechanism of electron transport. The systems studied include a molecular photo switch and polycyclic aromatic molecules.
Methodology and Applications

Optimization of atom centered potentials for the description of weak interactions in DFT: Extending the library to first row elements

Maurício D. Coutinho-Neto, I-Chun Lin, O. Anatole von Lilienfeld, Ivano Tavernelli, Michele Cascella, and Ursula Rothlisberger

Ecole Polytechnique Fédérale de Lausanne (EPFL), Laboratoire de Chimie et Biochimie Computationelle, CH-1015 Lausanne, Switzerland

The lack of proper description of dispersion forces in DFT when using generalized gradient corrected functionals is addressed by using dispersion calibrated atom centered nonlocal potentials (DCACPs). In this scheme [1-2] a nonlocal potential is constructed from individually calibrated components for each atom. The aim of this communication is three fold: a) to present an improved procedure for obtaining the parameters needed for determining the DCACPs that ensure a better description of the long range behavior while keeping the previously employed simple functional form; b) to present transferability studies for newly determined parameters for first row elements (Boron, Oxygen, Nitrogen, Fluorine), and c) to investigate the dependency of the DCACPs on the underlying xc-functional being used. To this end DCACPs obtained for rare gases using BLYP and PBE xc-functionals are compared. Particular emphasis is placed on the study of van der Waals complexes of simple aromatic heterocycles $(\mathrm{O}, \mathrm{N})$. The proper consideration of weak interactions will surely improve the first principles description of biomolecules where these effects are of major importance such as in DNA.

The simple and effective functional form of the DCACPs warrants their use within a first principles molecular dynamics framework with negligible additional computational cost.

[1] O.A. von Lilienfeld, et. al., Phys. Rev. Lett., 2004, 93, 153004.

[2] O.A. von Lilienfeld, et. al., J. Chem. Phys., 2005, 122, 14113.
Methodology and Applications

Average density self-interaction correction: application to molecular systems

$\underline{\text { Ilaria Ciofini }}{ }^{1}$, Carlo Adamo ${ }^{1}$ and Henry Chermette ${ }^{2}$

Ecole Nationale Supérieure de Chimie de Paris, Laboratoire d'Electrochimie et Chimie Analytique, 11 rue P. et M. Curie, F-75231 Paris Cedex 05, France and Laboratoire de Chimie Physique Théorique Bat. 210

Université Claude Bernard Lyon I, 43 Boulevard du 11 Novembre 1918 69622 Villeurbanne Cedex, France

The self-interaction error stems directly from the approximate nature of the exchange correlation potential: contrary to the HF, the self-interaction energy contained in the classical Coulomb energy of the Kohn-Sham (KS) Hamiltonian is only partially cancelled by the approximated exchangecorrelation contribution. Recently, we implemented the model, proposed by C. Legrand, E. Suraud and P.G. Reinhard [1], based on an average density self-interaction correction (ADSIC), where both exchange and Coulomb contributions are screened by a fraction of the electron density. The method provides results in good agreement with the experimental values for the prediction of IP in the case of strongly conjugated systems [2-3]. Here, we present its application to larger conjugated systems (such as polyethylene oligomers) for the prediction of structural and electronic properties. The performances of the method are shown to be comparable to those of more sophisticated (orbital dependent) self interaction corrections and the results obtained are in good agreement with the experimental data [4].

[1] E. Legrand, P.G. Suraud, J. Reinhard Phys. B 2002, 35, 1115I.

[2] I. Ciofini, H. Chermette, C. Adamo Chem. Phys. Lett. 2003, 380, 12

[3] I. Ciofini, C. Adamo, H. Chermette Phys. Chem. 2005, 309, 67.

[4] I. Ciofini, C. Adamo, H. Chermette soumis 
Methodology and Applications

\section{Photophysical Behaviour and Photoinduced Intramolecular Electron} Transfer in Ru and Os Complexes: Insights from Theory

\author{
$\underline{\text { Ilaria Ciofini }}^{\mathrm{a}}$, Nathalie Bouet ${ }^{\mathrm{a}}$, Philippe P. Lainé ${ }^{\mathrm{b}}$ and Carlo \\ Adamo $^{\mathrm{a}}$
}

a Laboratoire d'Électrochimie et Chimie Analytique, École Nationale Supérieure de Chimie de Paris, 11 rue P. et M. Curie, F-75231 Paris Cedex

05 France; b Laboratoire de Chimie et Biochimie Pharmacologiques et, Toxicologique Université René Descartes, 45 rue des Saints Pères, F-75270, Paris Cedex 06, France

The photophysical behavior of a series of $\mathrm{Ru}(\mathrm{II})$ and $\mathrm{Os}(\mathrm{II})$ complexes has been analyzed using a modern and effective approach to density functional theory (DFT). The Time Dependent-DFT approach was used to interpret the electronic spectra of a series of inorganic dyads, P-A (see Figure), expected to undergo intramolecular photoinduced ET to form charge separated state, of pivotal interest in many applications, including solar energy conversion and molecular electronics [1-2]. In all the considered cases, besides the noticeable quantitative agreement between computed and experimental absorption spectra, our results allow to clarify, by first principles, the nature of the excited states. The interplay of the electronic and geometrical coupling between the acceptor moiety and the photosensitizer was analyzed.
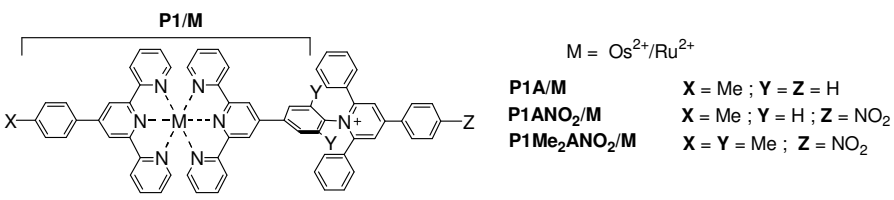

[1] I. Ciofini, P. P. Lainé, F. Bedioui, C. Adamo, J. Am. Chem. Soc. 2004, 126, 10763.

[2] N. Bouet, I. Ciofini, P. P. Lainé, F. Bedioui, C. Adamo work in progress

Methodology and Applications

LDF and OEP approach to multiplet energy calculation of 3d-transition metal atoms

\section{Chikatoshi Satoko and Nobukuni Hamamoto}

Department of Integrated Sciences of Physics and Biology Nihon University, 3-25-40 Sakurajousui,Setagaya-ku,Tokyo 156-8550,Japan

In recent years, the first principle calculation has been performed by the local density functional method(LDF method) where one must write the wavefunction as a single Slater determinant function. However,multiplet states of a transition metal atom are not always described as a single Sleter determinant function. So we study how the LDF can be applied to the multiplet state energy analysis.

Firstly, to calculate the multiplet state energies of a transition metal atom,we write the effective Hamiltonian in a second quantization as the sum of one body interactions and two body interactions which are estimated from the various methods such as Hartree-Fock and LDF methods. We are advancing these effective Hamiltonian method to study the electron correlation of the 3 d-transition metal ions.

Secondly, we calculate the optimized effective potential using the total energy functional of the single electron orbitals. The OEP is defined as the potential $V(r)$ under the stationary point of the functional derivative of the total energy, $E$, that is , $\delta E / \delta V(r)=0$. For the multiplet $|L S\rangle$ of transition metal atoms, the total energy is expressed as the linear combination of the Slater intergals $F^{k}(i j), G^{k}(i j)$ in the following way:

$$
E=\sum_{i} q_{i}^{L S} I\left(n_{i} l_{i}\right)+\frac{1}{2} \sum_{i j k} a_{i j k}^{L S} F^{k}(i j)+\frac{1}{2} \sum_{i j k} b_{i j k}^{L S} G^{k}(i j) .
$$

The OEP potential $V(r)$ solved from the OEP integral equation can be approximately written as the linear combionation of the separated OEP potentials corresponding to the Slater intergral $F$ and $G$ terms. ${ }^{1}$ We will discuss the LDF potential for the multiplet energy calculation of the transition metal atoms.

[1]N.Hamamoto and C.Satoko, J.Phys.B:At.Mol.Phys., 2004, 37, 4309.
Methodology and Applications

\section{Electron Propagator Approaches to DFT Reactivity Indices}

\author{
Junia Melin and J. V. Ortiz \\ Department of Chemistry, Kansas State University, Manhattan, Kansas 66506-3701.
}

An application of electron propagator formalism to calculate reactivity indexes of different chemical systems is presented. Solving the Dyson equation implicitly considers an electronic transition, and its eigenfunctions are FeynmanDyson amplitudes (or Dyson orbitals). Using Koopmans' theorem, Dyson orbital energies can be related to ionization potentials and electron affinities, therefore global reactivity indexes, such as chemical potential, hardness and electrophilicity can be directly obtained. Local reactivity indices, such as the Fukui function and local softness, are related to the sensitivity of ionization potential and electron affinity to external perturbations. Consequently, local reactivity indices can also be computed using the electron propagator formalism.

The approximations inherent to the electron propagator formalism are associated with the nonlocal self-energy operator. The simplest quasiparticle methods assume that off-diagonal elements of self-energy matrix can be neglected. The resulting Dyson orbitals are identical to canonical molecular orbitals, except for a correction factor (pole strength), but the corresponding orbital energies are correlated. Non-diagonal approximation techniques have been also implemented. Here orbital mixing is allowed, the orbital relaxation effects are included in the resulting Feynman-Dyson amplitudes.

This study accounts for an exploration of the behavior of reactivity indexes under diagonal and non-diagonal approximations of the self-energy matrix. Results are compared with standard calculations of these indexes within DFT. Since Dyson orbital energies include correlation and orbital relaxation effects, our goal is to analyze chemical systems where orbital relaxation is important

1. J. V. Ortiz in Computational Chemistry: Reviwes of Current Trends Vol. 2, J. Leszczynski, World Scientific, Singapore, 1997), p 1.

Methodology and Applications

\section{Gas-phase structure determination: how DFT can help}

\section{Sarah L. Hinchley and David W. H. Rankin}

\section{School of Chemistry, University of Edinburgh, West Mains Road,} Edinburgh, EH9 3JJ, UK

Structures determined in the gas phase using gas electron diffraction (GED) are free from intermolecular interactions that can affect solid-state structures. As a result, quite different structures can be obtained by solidstate and gas-phase investigations of the same molecule. The gas-phase experiment is used to elucidate geometric parameters for a wide range of compounds, from small symmetric organic systems to large, asymmetric inorganic molecules. Data can also be used by computational chemists to calibrate new code and programs. In turn, for large, complicated systems, GED makes excellent use of computed parameters as extra data in the process of structure determination.

This development, known as SARACEN ${ }^{1}$ (Structure Analysis Restrained by $\boldsymbol{A} b$ initio Calculations for Electron diffractioN), has enabled many, some very complicated, systems to be studied that would previously have been inaccessible. A consequence of this is that many transition metal systems can now be studied, for which traditional ab initio methods often fail when predicting parameters that include the transition metal itself. A classic example of this is ferrocene, ${ }^{2}$ for which both HF and MP methods fail to model the Fe-ring distance, whilst DFT methods give good agreement with experimental values.

This poster presents examples of systems containing heavy elements for which DFT has proved invaluable in the structure determination process.

[1] See, for example, N. W. Mitzel and D. W. H. Rankin, J. Chem. Soc., Dalton Trans. 2003, 19, 3650.

[2] A. Haaland and J. Nielson, Acta Chem. Scand. 1968, 22, 2653; W. Klopper and H. P. Lüthi, Chem. Phys. Lett. 1996, 262, 546; H. Koch, P. Jørgensen and T. Helgaker, J. Chem. Phys. 1996, 04(23), 9528. 
Methodology and Applications

$N$-representability of $p$-order reduced density matrix

Thierry Deutsch

DRFMC/SP2M/L_Sim, CEA-Grenoble - France

The two-order reduced density matrix contains all information useful to calculate the properties of a system of $N$ fermions. In 1960, Coulson [1] launched the idea, called Coulson's challenge by Coleman [2], to replace the many-body wavefunction with the two-order reduced density matrix in quantum mechanics calculations. This could reduce dramatically the complexity of the calculations and avoid some drastic approximations. The problem was that the necessary and sufficient conditions to have a $p$-order reduced density matrix which represents a system of $N$ fermions were not established at the exception of the first-order reduced density matrix. Since 1960, many attempts have been made and some sufficient or necessary conditions have been obtained.

The necessary and sufficient conditions to have a $N$-representable $p$ order reduced density matrix, i.e. corresponding to a wavefunction of $N$ fermions where $p$ is an integer between 1 and $N$, will be proposed in this presentation. Exact calculations could now be tractable for fermions without any many-body wavefunction.

[1] Coulson, Rev. Mod. Phys, 1960, 32, 170.

[2] Coleman and Yukalov, Reduced Density Matrices. Coulson's challenge, 2000, Springer
Methodology and Applications

CP2K/QUICKSTEP: Fast and accurate density functional calculations

Matthias Krack

Computational Science, Department of Chemistry and Applied Biosciences, ETH Zurich, USI-Campus, via G. Buffi 13, CH-6900 Lugano, Switzerland email: krack@phys.chem.ethz.ch

QUICKSTEP $^{1}$ is an implementation of the Gaussian and plane waves (GPW) method $^{2}$ which is based on the Kohn-Sham formulation of density functional theory (DFT). Gaussian basis sets and pseudopotentials optimized for the use with QUICKSTEP are presented to show its accuracy. The efficiency of QUICKSTEP allows to use large Gaussian basis sets for systems up to 3000 atoms. In this way atomistic and molecular simulations of solid state, liquid, molecular and biological systems can be performed. QUICKSTEP is designed to run efficiently on both supercomputers and PCs. The QUICKSTEP code is part of the freely available program package $\mathrm{CP} 2 \mathrm{~K}^{3}$.

[1] J. VandeVondele, M. Krack, F. Mohamed, M. Parrinello, T. Chassaing, J. Hutter, Comput. Phys. Commun., 2005, 167, 103

[2] G. Lippert, J. Hutter, M. Parrinello, Mol. Phys., 1997, 92, 477

[3] http://cp2k.berlios.de
Methodology and Applications

Time Dependent Density Functional Theory for molecular photoionization with non-iterative algorithm and multicenter B-spline basis set: implementation and applications

\section{Stener, G. Fronzoni and P. Decleva}

Dipartimento di Scienze Chimiche, Università di Trieste, Via L. Giorgieri 1 I-34127 TRIESTE ITALY

and Consorzio Interuniversitario Nazionale per la Scienza e Tecnologia dei Materiali, INSTM, Unita' di Trieste

and INFM DEMOCRITOS National Simulation Center, Trieste, Italy

A new direct (non-iterative) algorithm to solve the TDDFT equations for molecular photoionization is proposed and implemented, using a multicentric basis set expansion of B-spline functions and complete exploiting of the molecular point group symmetry. The method has been applied to study the photoionization and photoabsorption dynamics of $\mathrm{CS}_{2}$, $\mathrm{C}_{6} \mathrm{H}_{6}$ and other molecules: the results confirmed the expectation of large screening effects in $\mathrm{CS}_{2}$. For $\mathrm{C}_{6} \mathrm{H}_{6}$ the screening effects have been found to play a minor role than in $\mathrm{CS}_{2}$, however also in this case the quality of the final results is definitely improved. The method has proven suitable to study with confidence molecules of medium size, and there is still room for further improvement working on more elaborate treatment of the exchange correlation functional.
Methodology and Applications

\section{Comparison of different relativistic unrestricted two-component approaches for calculations of EPR parameters}

\author{
$\underline{\text { Michal Repiský }}{ }^{1}$, Vladimir G. Malkin ${ }^{1}$, Irina Malkin ${ }^{2}$, Olga L. Malkina ${ }^{1}$, \\ Martin Kaupp ${ }^{2}$, Stanislav Komorovský ${ }^{1}$

\footnotetext{
${ }^{1}$ Institute of Inorganic Chemistry, Slovak Academy of Sciences, Dúbravská cesta 9, SK-84536, Bratislava, Slovak Republic

${ }^{2}$ Institut für Anorganische Chemie, Universität Würzburg, Am Hubland, D-97074 Würzburg, Germany
}

Relativistic effects play an important role in heavy element chemistry. This is especially visible in EPR spectroscopy because all the operators responsible for those magnetic interactions can be naturally derived only from a relativistic theory. While the calculation of EPR parameters using the Dirac 4-component equation is a rather difficult task, different 2-component approximations have been suggested. In the present work, we implemented and compared 2-component relativistic Douglas-Kroll-Hess and ZORA approaches with the Dirac method. All considered methods are based on Density Functional theory in its unrestricted non-collinear implementation. Using three SCF calculations with orthogonal orientations of total spin, the full g-matrix and HFS tensor are obtained. The new methods remove effectively the dilemma existing up to now, regarding the simultaneous inclusion of spin polarization and higher-order spin-orbit effects in calculations of $\mathrm{g}$ - and HFS tensors. Moreover, it is straightforwardly applicable to higher than doublet spin multiplicities. 
Methodology and Applications

\section{Visualization of nuclear spin-spin coupling pathways by real-space functions}

\author{
Olga L. Malkina, Vladimir G. Malkin
}

Institute of Inorganic Chemistry, Slovak Academy of Sciences,

Dubravska cesta 9, SK-84236 Bratislava, Slovakia

Indirect nuclear spin-spin coupling constants are amongst the most important magnetic resonance parameters, invaluable in establishing molecular structure from NMR spectroscopy. Their detailed understanding in terms of molecular and electronic structure is thus of central importance in many fields of research and has been pursued since the beginnings of NMR spectroscopy more than 50 years ago. For example, questions of "through-space" versus "through-bond" mechanisms, the coupling pathways in polycyclic systems and, most recently, spin-spin couplings through hydrogen bonds have stimulated intensive discussions. Different types of analyses were used to obtain further information about different contributions to spin-spin coupling in terms of localized or canonical molecular orbitals (MOs). While these approaches can provide very useful insight, their conclusions depend on MO transformations and are thus strongly model-dependent: different MO schemes may lead to conflicting interpretation.

Here we will show that a model-independent description of spin-spin coupling is possible, based on real-space functions in three-dimensional space, which is appropriate for both localized and delocalized bonding situations $[1,2]$. The newly proposed and implemented real-space functions allow unprecedented insights into the pathways of indirect nuclear spin-spin couplings. In particular, this method allows one to distinguish through-space and through-bond interactions.

[1] O.L. Malkina, V.G. Malkin, Ang. Chemie Int. Ed., 2003, 42, 4335.

[2] O.L. Malkina, "Interpretation of indirect nuclear spin-spin coupling constants", in "Calculation of NMR and EPR Parameters: Theory and Applications", Eds. M. Kaupp, M. Bühl, V.G. Malkin, Wiley, Weinheim, 2004.

Methodology and Applications

Virial and scaling relations for the exchange-correlation energy functional in the extended constrained-search theory

Katsuhiko Higuchi $^{1}$ and Masahiko Higuchi ${ }^{2}$

Graduate School of Advanced Sciences of Matter ${ }^{1}$ Hiroshima University, Higashi-Hiroshima 739-8530, Japan

Department of Physics, Faculty of Science ${ }^{2}$

Shinshu University, Matsumoto 390-8621, Japan

The extended constrained-search (ECS) theory has recently been proposed so as to choose the electron density plus arbitrary physical quantities as basic variables. ${ }^{1}$ In order to perform the actual energy-band calculation within the ECS theory, a tractable form of the exchange-correlation energy functional is indispensable, i.e., some approximation has to be made on the exchange-correlation energy functional.

One of the strategies for developing such an approximate functional is to utilize some exact relations as sum rules which should be satisfied for the exchange-correlation energy functional. Along the strategy, we discuss the virial theorem of the ECS theory. The result gives a sum rule for the exchange-correlation energy functional. The present virial relation is expected to be a good starting point toward the approximation of the exchange-correlation energy functional, since the density-moment expansion for the conventional exchange-correlation energy functional is based on its virial relation.

Furthermore, scaling requirements for the exchange-correlation energy functional are also derived in case where the paramagnetic current is chosen as the basic variable. These sum rules can be utilized to determine the expansion coefficients of the exchange-correlation energy functional. We know that such an approach had already been used in the development of GGA exchange-correlation energy functional.

[1] M. Higuchi and K. Higuchi, Phys. Rev. B, 2004, 69, 035113.
Methodology and Applications

"Double local hybrid" potentials: construction and performance in DFT calculations of magnetic-resonance parameters

\section{Alexei V. Arbuznikov, Martin Kaupp and Hilke Bahmann}

Institut für Anorganische Chemie

Universität Würzburg, Am Hubland, 97074 Würzburg, Germany

The concept of a local hybrid functional [1] is a flexible promising generalization of traditional hybrid exchange-correlation functionals: instead of being a constant, the amount of the exact exchange admixture becomes a function of position vector (local mixing function (LMF)).

We have recently demonstrated that very encouraging results for nuclear shielding constants [2] and electronic g-tensors [3] can be obtained with traditional hybrid functionals provided that they are implemented in a fully self-consistent way, i.e. with local and multiplicative Kohn-Sham potentials derived from them via a reasonable approximation [4] to the optimized effective potential (OEP). In this work we apply a similar approach to derive localized potentials from local hybrid functionals, denoted "double local hybrid" (DLH) potentials.

A critical point is the choice of LMF. Along with the originally proposed [1] ratio of the Weizsäcker kinetic energy density to local kinetic energy density, we examine other kinds of LMF.

The resulting DLH potentials are evaluated, e.g., in calculations of nuclear shieldings for main-group molecules and g-tensors for $3 \mathrm{~d}$ transitionmetal complexes.

[1] J. Jaramillo, G. E. Scuseria, M. Ernzerhof, J. Chem. Phys., 2003, 118, 1068.

[2] A. V. Arbuznikov, M. Kaupp, Chem. Phys. Lett., 2004, 386, 8.

[3] A. V. Arbuznikov, M. Kaupp, Chem. Phys. Lett., 2004, 391, 16.

[4] F. Della Sala, A. Görling, J. Chem. Phys., 2001, 115, 5718.

Methodology and Applications

P36

On the Applicability of DFT-based Reactivity Descriptors to Kinetically and Thermodynamically Controlled Reactions

Karen Hemelsoet, ${ }^{1}$ Veronique Van Speybroeck, ${ }^{1}$ Damian Moran, ${ }^{2}$ Leo Radom ${ }^{2}$ and Michel Waroquier ${ }^{1}$

Center for Molecular Modelling, ${ }^{1}$ Laboratory of Theoretical Phyisics, Ghent University, Proeftuinstraat 86, 9000 Gent, Belgium School of Chemistry, ${ }^{2}$ University of Sydney, Sydney, NSW 2006, Australia

The addition of carbon-centered radicals to a variety of unsaturated molecules has been studied using DFT-based reactivity descriptors [1] and reliable kinetic information from high-level ab initio computations [2]. The hard and soft acids and bases (HSAB) principle has been used to order species with

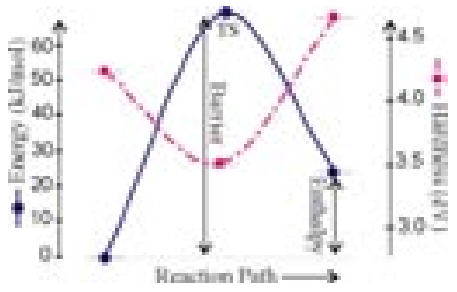
similar chemical reactivity (global descriptors) and to address the radical addition site-selectivity (local descriptors). Reactivity descriptors only provide information on the onset of a reaction, hence they perform well for kinetically controlled reactions, but tend to fail for thermodynamically controlled processes like many radical addition reactions. A better understanding of correlations between reactivity descriptors on the one hand and kinetic and thermodynamic results on the other, is obtained by studying the differences in the softness, the reaction barriers and the reaction enthalpies [3,4]. As an extension of this approach, the correlation with the Evans-Polanyi-Semenov relationship is studied.

[1] Geerlings P. et al. Chem. Rev. 2003, 103, 1793

[2] Fischer H. and Radom L. Angew. Chem. Int. Ed. 2001, 40, 1340

[3] Hemelsoet K. et al. J. Phys. Chem. A 2004, 108, 7281

[4] Van Cauter K. et al. Int. J. Quantum Chem. 2005, 102, 454 
Methodology and Applications

\section{An Evaluation of the Influence of Level of Theory on DFT-based Reactivity Descriptors}

Karen Hemelsoet, ${ }^{1}$ Veronique Van Speybroeck, ${ }^{1}$ Damian Moran, ${ }^{2}$ Leo Radom $^{2}$ and Michel Waroquier ${ }^{1}$

Center for Molecular Modelling, ${ }^{1}$ Laboratory of Theoretical Phyisics, Ghent University, Proeftuinstraat 86, 9000 Gent, Belgium School of Chemistry, ${ }^{2}$ University of Sydney, Sydney, NSW 2006, Australia

The success of DFT-based reactivity indicators can be found in both their low computational cost and their applicability to a broad range of chemical species [1,2]. Global indicators, which describe the general reactivity of chemical systems, are used to construct reactivity sequences, while local

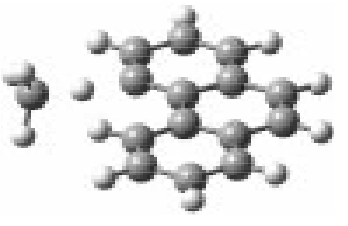
descriptors address reaction site-selectivity. In the current investigation, DFT-based reactivity descriptors have been computed using different basis sets and quantum chemical methods in combination with the Mulliken, Löwdin, CHELPG, RESP, Hirshfeld and NPA population schemes. Hydrogen abstraction from benzene and larger polyaromatic hydrocarbons (PAHs) by methyl radical has been used as a model system for methodological evaluations, as an earlier B3LYP/6-311G(d,p), showed a correlation between the reaction barriers and the local indicators [3]. Comparing PAH local softness differences and energy barriers calculated at different levels of electronic structure theory shows that the choice of population scheme is particularly important to descriptor performance.

[1] Parr R. G. and Yang W. Density-Functional Theory of Atoms and Molecules, Oxford Science Publications, Oxford, England, 1988

[2] Geerlings P., et al. W. Chem. Rev. 2003, 103, 1793

[3] Hemelsoet K., et al. J. Phys. Chem. A 2004, 108, 728

Methodology and Applications

Polarization Consistent Basis Sets.

Frank Jensen

Department of Chemistry

SDU, Odense, Denmark

Polarization consistent basis sets are Gaussian basis sets optimized for density functional calculations [1,2]. Some recent developments and results will be presented.

[1] F. Jensen J. Chem. Phys. 116 (2002) 7372

[2] F. Jensen, T. Helgaker J. Chem. Phys. 121 (2004) 3462
Methodology and Applications

\section{Extension of Sham-Schlüter equation}

Yasuteru Shigeta and Kimihiko Hirao

Department of Applied Chemistry, School of Engineering, The University of Tokyo, Hongo, Tokyo 113-8656, Japan.

It is well-known fact that the Kohn-Sham density functional theory (KS-DFT) often gives well description for the ground state energy and properties at least qualitatively. Nevertheless the results depend on the choice of an exchange-correlation energy functional whose exact form is still unknown. In order to construct the exact functional, a relationship between the Green's function (GF) method and KS-DFT has been investigated for three decades, because the Dyson equation has quite similar form to that the KS equation does. Conspicuous differences between these methods are that the exchange-correlation potential in the $\mathrm{KS}$ equation is local and energy-independent and is a functional of electron density. On the other hand, the self-energy in the Dyson equation is energy-dependent and has generally non-local potential. Sham and Schlülter have obtained an equation to derive a local and energy-independent potential from the GF formalism. Later Casida has proved that the Sham- Schlülter (SS) equation is regarded as one of the optimized effective potential (OEP) methods for GF. Very recently one of us has applied the SS equation to the multi-band superconductivity at finite temperature $[1,2]$. In this line, it is intrinsically necessary to construct non-local potential for the anomalous states.

In this work, we derive a non-empirical and (non-) local OEP using the SS equation. We then generalize the SS equation to those of temperature, Keldysh, and high order GFs starting from several consistency condition between exact and quasi-particle properties such as total energy, charges, density matrices, and so on.

[1] Y. Shigeta, Int. J. Quantum Chem., 2005, 101, 774.

[2] Y. Shigeta, K. Hirao, Phys. Rev. B, in preparation.

Methodology and Applications

P40

\section{Ab initio iterative CASCI-DFT approach}

Shusuke Yamanaka ${ }^{1}$, Koichi Kusakabe $^{2}$, Kazuto Nakata $^{1}$, Ryo Takeda ${ }^{1}$, Toshikazu Takada $^{3}$, Haruki Namura ${ }^{4}$, and Kizashi Yamaguchi ${ }^{1}$

1 Graduate School of Science, Osaka University. Machikaneyama 1-1, 5600043, Toyonaka, Osaka Japan.

2 Graduate School of Engineering Science, Osaka University. Machikaneyama, 1-3, 560-8531, Toyonaka, Osaka Japan.

3 NEC Corporation. Miyukigaoka, 305-8501, Tsukuba, Ibaraki, Japan

4 Institute for Protein Research, Osaka University, Yamadaoka 3-2, 5600871, Suita, Osaka Japan.

Despite its remarkable success, Kohn-Sham DFT with available exchange-correlation functional does not work well for systems exhibiting multireference (MR) character. For such a reason, a new class of electronic structure theory combining the complete-active-space (CAS) WFT with DFT, so-called "CAS-DFT" approach has been developed during the past decade [1,2]. The efforts of the developers are mainly devoted to resolve (i) how to avoid double counting problem of electron correlations and (ii) how to define the spin-polarizability of CAS wavefunction for singlet radicals, and indeed several approaches to work out (i) and (ii) problems are proposed [1,2].

On the other hands, Kusakabe proposed the use of interacting reference system in DFT, leading to the extended KS theory, previously [3]. We here integrate the concept of EKS and CAS-DFT methods into ab initio iterative CASCI-DFT method, where the CASCI wavefunction is used as a reference system with effective DFT potential constructed by CAS-DFT [1,2]. This approach is promising from the viewpoint of both of computational costs and results. The sample calculations and future directions are presented.

[1] B. Miehlich, H. Stoll, A. Savin, Mol. Phys. 91 (1997) 527;J. Grafenstein, D. Cremer, Chem. Phys. Lett., 316 (2000) 569.

[2] R. Takeda, S. Yamanaka, K. Yamaguchi, Chem. Phys. Lett. 366 (2002) 321; Int. J. Quantum Chem. 96 (2004) 463.

[3] K. Kusakabe, J. Phys. Soc. Jpn., 70 (2001) 2038; K. K. to be published. 
Methodology and Applications

\section{Hirshfeld Division as Diagnostic Tool of Atoms-in-Molecules}

\section{Roman F. Nalewajski, Elżbieta Broniatowska}

Faculty of Chemistry, Jagiellonian University, R. Ingardena 3, 30-060 Cracow, Poland

The information theory provides a solid foundation of Hirshfeld's partitioning of the molecular density into constituent Atoms-in-Molecules (AIM). The density of displacements of the Shannon entropy for the Hirshfeld constituent atoms is compared with their density difference function for selected small molecules [1, 2]. The one-electron Hirshfeld $(\mathrm{H})$ and two-electron stockholder (S) divisions of the molecular density are summarized. The one-electron densities of the $\mathrm{H}$ and $\mathrm{S}$ AIM obtained from UHF calculations are compared for several diatomics (e.g. $\mathrm{H}_{2}, \mathrm{LiH}, \mathrm{HF}, \mathrm{N}_{2}$ ). Similar analysis is carried out for the UHF twoelectron density corrected about the correlation density obtained from MP2 method. The stockholder atoms arising from two-electron density for $\mathrm{H}_{2}$ and $\mathrm{LiH}$ exhibit a higher degree of a cylindrical polarization around bond axis, in the direction of the bond partner, and more bonding character. In the cases of $\mathrm{HF}$ and $\mathrm{N}_{2}$ no dramatic difference between these two distributions are observed.

[1] R. F. Nalewajski, E. Broniatowska, J. Phys. Chem. A, 2003, 107, 6270.

[2] R. F. Nalewajski, E. Świtka, A. Michalak, Int. J. Quantum Chem., 2002, 87,198 .

[3] R. F. Nalewajski, E. Broniatowska, J. Phys. Chem. A, in preparation.
Methodology and Applications

P42

\section{Implications of Symmetry Rules for the Aromaticity of Inorganic Clusters}

\section{Clémence Corminboeuf, R. Bruce King, Paul v. R. Schleyer}

Department of Chemistry and Center for Computational Chemistry, University of Georgia, Athens, Georgia 30602

Isoelectronic clusters with the same basic cage structures may exhibit surprisingly different magnetic properties. For instance, nucleusindependent chemical shift[1] (NICS) values, computed at cluster centers, may range considerably in magnitude and even change from diatropic to paratropic. The same is true for dissected canonical molecular orbital contributions to the total NICS values, computed by the GIAO method. This work proposes and validates the use of symmetry selection rules to predict the overall magnetic behavior of inorganic clusters.[2] The magnitude of paratropic (deshielded, down-field shifted) CMO-NICS[3] contributions to total NICS values also is clarified by these rules. Thus, HOMO-NICS values are paratropic only if the HOMO $\rightarrow$ LUMO rotational transition is allowed by symmetry selection rules derived from group theory. High symmetry inorganic cages are likely to exhibit spherical aromaticity if the $\mathrm{HOMO} \rightarrow$ LUMO rotational transition is forbidden by these symmetry rules.

[1] Schleyer, P. v. R.; Maerker, C.; Dransfeld, A.; Jiao, H.; Eikema Hommes, N. J. R. J. Am. Chem. Soc. 1996, 118, 6317.

[2] Corminboeuf, C.; King, R. B.; Schleyer, P. v. R. submitted to J. Chem. Theory Comput.

[3] Heine, T.; Schleyer, P. v. R.; Corminboeuf, C.; Seifert, G.; Reviakine, R.; Weber, J. J. Phys. Chem. A 2003, 107, 6470.
Methodology and Applications

\section{Multi-Configuration Optimized Effective Potential Method}

\section{Martin Weimer and Andreas Görling}

Lehrstuhl für Theoretische Chemie, Universität Erlangen-Nürnberg Egerlandstrasse 3, 91058 Erlangen, Germany

Conventional Density Functional Methods fail to describe correctly correlation and therefore are unable to describe energy and symmetry simultaneously correctly in bond-breaking or twisting of molecule fragments against each other.

In order to solve these shortcomings and with the long-term objective developing an applicable orbital-dependent description of exchange and correlation, a multi-configuration optimized effective potential (OEP) approach is presented. It is technically related to the open-shell localized Hartree-Fock method (Ref.1-3), an effective exact-exchange Kohn-Sham approach for open-shell systems. In contrast to other OEP methods including the open-shell localized Hartree-Fock wavefunction the model wavefunction in the presented approach is neither a single determinant nor a single configuration state function but a linear combination of configuration state functions. The total electronic energy can be considered as a functional of an effective local multiplicative potential, the orbitalgenerating potential, which is determined by an OEP equation. This OEP potential, a state- and thus orbital-dependent potential, covers beside exchange effects also correlation effects, namely static correlation. Exemplary results for small and medium sized molecular systems are presented focusing on potential curves of the dissociation and twisting of molecular fragments.

\section{References}

1. A. Görling, Phys. Rev. Lett. 85, 4229 (2000).

2. F. Della Sala and A. Görling, J. Chem. Phys. 115, 5718 (2001).

3. F. Della Sala and A. Görling, J. Chem. Phys. 116, 5374 (2002).
Methodology and Applications

Novel properties of the Kohn-Sham exchange-correlation potential for open systems: Application to the two-dimensional electron gas

\section{S. Rigamonti, F. A. Reboredo*, C. R. Proetto}

Centro Atómico Bariloche and Instituto Balseiro, Av. E. Bustillo Km. 9.5, 8400 S. C. de Bariloche, Argentina

* Lawrence Livermore National Laboratory - Livermore, CA 94551, USA.

The properties of the Kohn-Sham (KS) exchange-correlation potential for open systems in thermodynamical equilibrium, where the number of particles is non-conserved, will be discussed [1]. A new accurate calculation method has been designed for the treatment of strongly correlated and inhomogeneous low-dimensional electronic systems [2]. The two-dimensional electron gas formed at the interface between two semiconductors ( $\left.\mathrm{GaAs} / \mathrm{Al}_{\mathrm{x}} \mathrm{Ga}_{1-x} \mathrm{As}\right)$ is used as an illustrative example. Results are obtained through an exact solution of the Optimized Effective Potential (OEP) method of DFT, at the exchange level. The main findings are that the KS exchange potential builds a significant barrier-like structure under slight population of the second subband, and that both the asymptotic value of the KS exchange potential and the inter-subband energy jumps discontinuously at the one subband $\rightarrow$ two subband transition. The results obtained in this system offer new insights on open problems of semiconductors, such as the band-gap underestimation and the band-gap renormalization by photo-excited carriers. First results will be presented for the KS correlation potential, as obtained with the OEP formalism, using a first-principle orbital and eigenvalue dependent energy correlation functional generated through the KS perturbation theory [3].

[1] S. Rigamonti, F. A. Reboredo, and C. R. Proetto, Europhys. Lett. 2005, 70, 116.

[2] F. A. Reboredo and C. R. Proetto, Phys. Rev. B 2003, 67, 115325; S. Rigamonti, F. A. Reboredo, and C. R. Proetto, Phys. Rev. B 2003, 68, 235309.

[3] A. Görling and M. Levy, Phys. Rev. B, 1993, 47, 13105. 
Methodology and Applications

A first-principles many-body calculation based on a new density-matrix functional theory

Koichi Kusakabe ${ }^{1}$, Masanori Takahashi ${ }^{1}$, Naoshi Suzuki ${ }^{1}$, Shusuke Yamanaka ${ }^{2}$ and Kizashi Yamaguchi ${ }^{2}$

Graduate School of Engineering Science, Osaka University ${ }^{1}$

1-3 Machikaneyama-cho, Toyonaka, 560-8531, Japan

Graduate School of Science, Osaka University ${ }^{2}$

1-1 Machikaneyama-cho, Toyonaka, 560-0043, Japan

We have recently developed a new density-matrix functional theory which shows uniqueness of the interaction parameter $U$ appearing in the extended Kohn-Sham equations (EKS)[1] via a proof given by a variational argument.[2] EKS is a rigorous version of the so-called LDA+U formulation of the density functional theory. In the $U$ term which is often cited as the Hubbard interaction in the literature, $U$ is determined as a functional of the density-density correlation function i.e. a second-order reduced density matrix. We proposed a criterion to determine this unique $U$ by reproducing the correlation function of the true ground state as well as the true density. In this presentation, we give a practical method to determine $U$ as well as the residual exchange-correlation functional starting from the CASCI-DFT calculation.[3] Accurate estimation of the fluctuation in a relevant open orbital is essential for our new EKS method. By obtaining the density-density correlation in each localized orbital or in each molecular orbital, we can give a criterion to determine optimized effective model for correlated electron systems.

[1] K. Kusakabe, J. Phys. Soc. Jpn. 2001, 70, 2038.

[2] K. Kusakabe, in "Introduction to the computational materials design", $\mathrm{H}$. Kasai ed. (Osaka University press) 2005.

[3] R. Takeda, S. Yamanaka, K. Yamaguchi, Chem. Phys. Lett. 2002, 366, 321; Int. J. Quant. Chem. 2004, 96, 463.

Methodology and Applications

DFT studies on Metal-Metal bonds -Application of Broken Symmetry DFT to Strong Correlation Systems-

Yasutaka Kitagawa, Shusuke Yamanaka, Mitsuo Shoji, Kenichi Koizumi, Mitsutaka Okumura, Takashi Kawakami and Kizashi Yamaguchi

Graduate School of Science, Osaka University

1-1 Machikaneyama, Toyonaka, Osaka 560-0043, Japan

A density functional theory (DFT) method has been applied to various systems and been showing successful results. However it still has an unsatisfactory point on strong electron correlation systems with degenerate valence orbitals. A metal-metal bonding is one of the typical cases of them. Ab initio calculations of those systems have been a difficult task because two different types of electron correlation effects, i.e. dynamical correlation and non-dynamical correlation, must be taken into consideration.

Recent trends of the computational approach for such strongly correlating systems are the use of broken symmetry (BS) DFT and BS hybrid DFT (HDFT) calculations. In this study, we examined how BS DFT (or BS HDFT) reproduced electron correlation effects in metal-metal bonding systems. In order to elucidate what UDFT (or UHDFT) expressed as correlation energies, total energy was decomposed into kinetic energy, nuclear-nuclear potential, nuclear-electron potential and electron-electron potential and they were compared with ones of UHF, CASSCF and so on. In addition, an energy gap between lowest spin state and highest spin state was also examined with respect to calculations of effective exchange intagrals $(\mathrm{J})$
Methodology and Applications

P46

Exact exchange Kohn-Sham and time-dependent DFT calculations for periodic systems

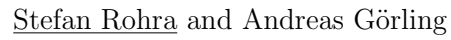

Lehrstuhl für Theoretische Chemie, Universität Erlangen-Nürnberg Egerlandstrasse 3, 91058 Erlangen, Germany

Density Functional Methods like LDA or GGA usually give nonsatisfiying results for periodic systems like an underestimation of the band gap in band structure calculations or the lack of excitonic effects in the calculation of optical properties. In Ref.[1,2] a plane-wave Kohn-Sham method, which contructs and uses the exact exchange potential was presented. The method yields improved band gaps for semiconductors.

For the calculation of electronic response properties within time-dependen DFT an exact-exchange kernel has recently been developed Ref.[3,4]. Here exact exchange KS and time-dependent DFT methods are applied to polyacetylene as a one-dimensional periodic system and various three dimensional semiconductors, calculating bandstructures, independent particle response functions and optical absorption spectra. The results are compared to those obtained with conventional potentials and kernels.

[1] M. Städele et al., Phys. Rev Lett., 792089 (1997).

[2] M. Städele et al., Phys. Rev B, 5910031 (1999).

[3] Y.-H. Kim and A. Görling., Phys. Rev B, 66035114 (2002).

[4] Y.-H. Kim and A. Görling., Phys. Rev Lett., 89096402 (2002).
Methodology and Applications

Coupling of electrons to RPA excitations in atoms

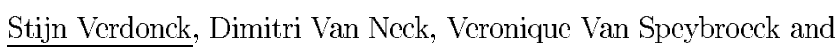
Michel Waroquicr

Ghent University

Contre for Molccular Modclling

Procftuinstraat 86, B-9000 Gcnt, Bclgium

The goal of this work is to develop a better understanding of elcetronic corrclations in finite systems, and to conncet DFT with woll-cstablishcd concepts in Grcen's function many-body perturbation theory. For a scrics of closcd-shell atoms we construct the coordinate-space HF orbitals. The continuum of the HF mean ficld is discretized in a controlled manncr ${ }^{1}$. Within this particle-hole $(p h)$ basis the RPA cquations are solved, taking both the direct and cxchange part of the ph-intcraction into account. As cxpected, the excited states remain rather pure $p h$ configurations, and the main collective effect is a change of the mean-ficld for the particle statcs into the ion-like potential obtained by cancelling the contribution of the relevant hole state to the HF potential ${ }^{2}$. Good agrcement is obscrved with the expcrimental ph spectrum of the ncutral atom. after shifting the RPA spectrum by an amount $\Delta=\varepsilon_{h}^{I I F}-\varepsilon_{h}^{e x p}$. The elcctron sclf-encrgy is then constructed by coupling the HF propagator to the RPA polarization. Solving the corresponding Dyson cquation leads, howcver, to ionization cnergies that scriously overshoot the shift necded to get from HF to the experimental values. Much better agrecment is obtained by coupling to the RPA polarization with only the dircet $p h$ interaction retained (which is cquivalent to the $G^{(C)} W^{(0)}$ approximation).

[1] D. Van Neck, K. Pcirs, M. Waroquicr, J. Chem. Phys., 2001, $115,115$.

[2] M. Ya. Amusia, Phys. Rep., 1996, 264, 7. 
Methodology and Applications

A new class of density functionals, based on the energy-dependence of the electronic self-energy

Dimitri Van Neck, Veronique Van Speybroeck and Michel Waroquier

Ghent University

Centre for Molecular Modelling

Proeftuinstraat 86, B-9000 Gent, Belgium

We present extensions of the conventional Kohn-Sham implementation of DFT. This is done in the framework of Green's function perturbation theory. The electronic spectral function can in general be decomposed in a (Landau-Migdal) quasiparticle (QP) part and a correlation background. Using the exact energy-weighted sum rules obeyed by the electronic spectral function it is shown that a modelling of the (small) background part in terms of the local density is sufficient to generate a single-particle self-consistency problem. The resulting one-electron energies and wave functions have a clear interpretation as quasiparticle properties (i.e. the ionization energies, electron affinities, and overlap functions between the $N$-electron ground state and the $N \pm 1$ eigenstates). The proposed modelling of the background part is based on the electronic spectral function calculated in various simple test svstems: light atoms with a selfconsistent evaluation of the second-order self-energy ${ }^{1}$, and with the $G^{(\mathrm{C})} W^{(\mathrm{C})}$ approximation ${ }^{2}$, and the electron gas in the $G W$ approach $^{3}$. The resulting single-particle self-consistency problem includes the effect of correlations, and leads to a simultaneous description of the total electronic energy and the ionization energy in a test set of closed-shell atoms.

[1] D. Van Neck, K. Peirs, M. Waroquier, J. Chem. Phys., 2001, 115, 15; K. Peirs, D. Van Neck, M. Waroquier, J. Chem. Phys., 2002, 117, 4095; K. Peirs, D. Van Neck, M. Waroquier, Phys. Rev. A, 2003, 115, 012505.

[2] S. Verdonck, D. Van Neck, V. Van Speybroeck, M. Waroquier, Poster contribution at this conference.

[3] Y. Dewulf, D. Van Neck, M. Waroquier, Phys. Rev. B, 2005, 71, accepted for publication.

Methodology and Applications51

Development of exchange-correlation functionals with the optimized effective potential method for the response properties of extended systems

\section{Myrta Grüning $^{1}$, Andrea Marini ${ }^{2}$, Angel Rubio ${ }^{3,1}$}

Donostia International Physics Center ${ }^{1}$ and Dpto. Fisica de Materiales, Facultad de Quimicas, Universidad del Pais Vasco ${ }^{3}$, 20018 San Sebastián - Spain

Dipartimento di Fisica, Università Roma "Tor Vergata"2, 00133 Rome - Italy.

Standard exchange-correlation (xc) functionals based on the local density approximation (LDA), or on gradient approximations, are in general not reliable for the response properties of extended system. In particular for optical absorption spectra of semiconductors and insulators the spectra are shifted towards lower energies and excitonic effects are not correctly taken into account.

In this study we try to clarify the impact of the approximations (both of the potential and the kernel) for the bad description of the optical spectra. With the optimized effective potential (OEP)[1] method we obtain an accurate approximation for the xc potential where the long range correlation is included as dynamical screening of the Coulomb interaction (in the GW approximation). The results with this potential and the LDA potential for the band structure of prototype systems are very close and suggest that - at least for this type of systems - the LDA xc potential gives reasonable results and the downward shift in the spectra is due to the approximation of the kernel.

We derive a static, non-local kernel with the proper long range behavior, using the common energy denominator approximation[2] for the OEP equation. We discuss the calculations of optical absorption spectra of semiconductors and insulators within this approximation.

[1] J. D. Talman, W. F. Shadwick, Phys.Rev.A, 1976, 14, 36.

[2] O. V. Gritsenko, E. J. Baerends, Phys.Rev.A, 2001, 64, 42506.
Methodology and Applications

\section{COSMO calculations with $\mathrm{DMol}^{\mathfrak{s}}$ revisited:} molecules, polymers and surfaces

\section{B. Delley}

Paul Scherrer Institute, Villigen CH-5232 Switzerland

The conductor-like screening model (COSMO) simulates the molecular response to a solvent by placing the molecule in a hypothetical metal wrapper. In this way the numerous degrees of freedom of the solvent are modelled. This results in a numerically much more expedient method to get solute properties than with first principles modelling of the solvent $[1,2]$. The response to the idealized solvent screening can be used as input to parametrized model calculations for real solvents [3]. Density functional theory calculations can be done for molecules [4] as well as for systems with periodic boundary conditions [5] within the same approach. First principles molecular dynamics studies can be done again within that approach [6].

$\mathrm{DMol}^{3}$-COSMO calculations have been generalized now for polymers, twodimensional periodic surfaces and even internal surfaces in bulk such as are typical for zeolites. For this purposes a redesigned algorithm for the discretized solvent accessible surface has been developed. Also, the electrostatics calculations have been generalized for the continuation of the surface with periodic boundary conditions. Comparisons of the old and the new method will be presented for molecules. Applications of the new method to a polymer and a surface will be given.

[1] A. Klamt, G. Schüürmann, J. Chem. Soc. Perkin Trans. 2, 799-805 (1993)

[2] J. Andzelm et al, J. Chem. Phys. 103, 9312 (1995)

[3] A. Klamt, J. Phys. Chem. 99, 2224 (1995)

[4] B. Delley J. Chem. Phys. 92, 508 (1990)

[5] B. Delley J. Chem. Phys. 113, 7756 (2000)

[6] R. Windiks and B. Delley, J. Chem. Phys. 119, 2481 (2003)

Methodology and Applications

P52

Molecular Dynamics with Quantum Transitions Using Time-Dependent DFT

Enrico Tapavicza, Ivano Tavernelli, Ursula Rothlisberger

Swiss Federal Institute of Technology, EPFL, CH-1015 Lausanne, Switzerland.

Many chemical reactions of organic molecules occur in electronically excited states and can only be described taking into account non-adiabatic effects. Time-dependent (TD) DFT [1] allows to calculate electronic spectra and nuclear forces for electronically excited molecules. There have been several approaches to include non-adiabatic effects in ab initio molecular dynamics codes based on surface hopping (SH) [2] or Ehrenfest dynamics [3].

Recently we presented a SH mechanism for TDDFT, based on Landau-Zener theory $[4,5]$, where the probability of switching excited state is computed from the nuclear forces at the avoided crossing between two electronic states [6].

Here we present a more general method, similar to the approach of Tully [7]. The non-adiabatic coupling is directly computed using an approximation for the excited state wavefunction.

The method has been implemented in the plane wave code CPMD $[8,9]$ and applied to intramolecular photoinduced electron transfer in organic model compounds. A comparision to the Landau-Zener method is presented.

[1] E. Runge, E.K.U. Gross, Phys.rev.Lett., 1984, 52, 997.

[2] I. Tavernelli, U. F. Rohrig, U. Rothlisberger, Mol. Phys., 2005, in press.

[3] N.L. Doltsinis, D. Marx, Phys.Rev.Lett., 2002, 88, 166402.

[4] L.D. Landau, Phys.Z.Sowjetunion, 1932, 2, 46.

[5] C. Zener, Proc.R.Soc., 1932, A137, 696.

[4] I. Tavernelli, E. Tapavicza, U. Rothlisberger, 2005, in preparation.

[5] J.C. Tully, J.Chem.Phys., 1990, 93, 1061.

[8] Car Parrinello Molecular Dynamics code, http://www.cpmd.org.

[9] J. Hutter, J.Chem.Phys., 2003, 118, 3928. 
Methodology and Applications

\section{Open molecular knots with three crossings. A general computational} approach.

\section{Christos Krinas ${ }^{1}$, Ioannis Demetropoulos ${ }^{1}$}

Department of Chemistry ${ }^{1}$

University of Ioannina, 45110 Ioannina, Greece

A general computational procedure is presented that could efficiently construct knotted structures with 3 crossings and open ends from scratch. Three classes of molecules are investigated: polyethylene, poly(ethylene oxides) and peptides. The stability of these complex molecular conformations is eventually examined using Density Functional Theory.

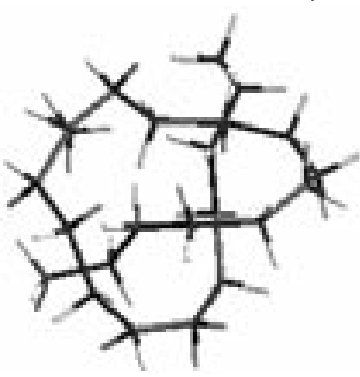
Flexible molecules if they are long enough they could entangle themselves and form knotted structures. We built a computer package that generates, examines and manipulates about 100 million structures with high probability of knottiness. The tightest knot for a polyethylene strand suggested to be 23 carbon atoms ${ }^{[1,2]}$. Torsion angles, bond angles and bond lengths of the knotted structures are nearly identical at both B3LYP/6-31(d,p) and PW91/6-31(d,p) level of theory. Figure presents the $\mathrm{C}_{23} \mathrm{H}_{48}$ at a knot shape. However this $3 \mathrm{D}$ molecular arrangement exhibits bond lengths and bond angles extremely stretched, up to values: $1.74 \AA$ and $144.9^{\circ}$ respectively, at B3LYP/6-31(d,p), although at MP2/6-31(d,p) level the corresponding values are $1.69 \AA$ and $144.6^{\circ}$. Similar results are reported for poly(ethylene oxides).

[1]. M.L. Mansfield, Macromolecules, 1998, 31, 4030.

[2]. A.M. Saitta, et al., Nature, 1999, 399, 46.

Methodology and Applications

Applications of the total energy bifunctional in studies on hydrogenbonded complexes.

\section{Ruslan Kevorkyants, Marcin Dulak and Tomasz Wesolowski.}

Department of Physical Chemistry

University of Geneva, 30 quai Ernest Ansermet, 1211 Geneva, Switzerland.

We report on the performance of local density approximation (LDA) and gradient-dependent generalized gradient approximation (GGA) functionals in variational calculations ${ }^{1}$ using the total energy bifunctional $E\left[\rho_{A}, \rho_{B}\right]$. These approximations are applied to exchange-correlation and non-additive kinetic energy components of $\mathrm{E}\left[\rho_{\mathrm{A}}, \rho_{\mathrm{B}}\right]$ for various hydrogen-bonded complexes (water-dimer, HCN-HF etc.). For these systems the interaction energies are computed and compared to benchmark ab initio interaction energies taken from the literature. In addition individual components of total energy bifunctional $\mathrm{E}\left[\rho_{\mathrm{A}}, \rho_{\mathrm{B}}\right]$ are analyzed. For considered complexes the interaction energies fall into the range of $1.5-7.5 \mathrm{kcal} / \mathrm{mol}$.

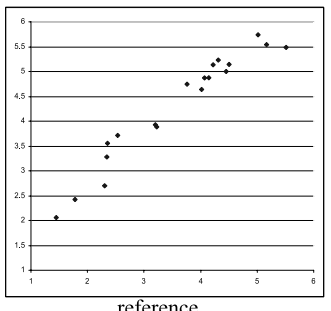

reference
Our previous studies showed that LDA leads to very good interaction energies only for systems with very small $\rho_{A} \rho_{B}$ overlap (e.g. $\mathrm{Ne}_{2}$ ) but it is not satisfactory in cases where the overlap is large ${ }^{2}$. Here we show that the GGA approximation allows one to extend applicability of the total energy bifunctional $\mathrm{E}\left[\rho_{\mathrm{A}}, \rho_{\mathrm{B}}\right]$ to hydrogen-bonded complexes (see picture).

[1] Wesolowski T.A., Warshel A., J.Phys.Chem., 97 (1993), p.8050.

[2] Wesolowski T.A., Tran F., J.Chem.Phys. 118 (2003), p.2072.
Methodology and Applications

Connection Between the Average Local Ionization Energy and the Fukui Function. Theory and Applications.

$\underline{\text { Soledad Gutiérrez-Oliva }}^{1}$, Pablo Jaque ${ }^{1}$, Felipe Bulat ${ }^{1}$, Alejandro Toro-Labbé $^{1}$, Jane S. Murray ${ }^{2}$ and Peter Politzer ${ }^{2}$

${ }^{1}$ Laboratorio de Química Teórica Computacional (QTC),

Facultad de Química, Pontificia Universidad Católica de Chile. ${ }^{2}$ Department of Chemistry, University of New Orleans, New Orleans, LA 70148, USA.

The average local ionization energy $\bar{I}(r)$ and the Fukui function $f(r)$ are conceptually independent measures of molecular reactive behavior, both quantities are expected to identify the same sites as most reactive toward electrophiles. In this work we use Janak's theorem to establish a link between $\bar{I}(r)$ and $f(r)^{1}$. Numerical applications confirm that both of these local properties convey similar information concerning some aspects of the reactive behavior of molecular systems.

[1] A. Toro-Labbé, P. Jaque, J. S. Murray, P. Politzer, Chem. Phys. Lett., 2005, 40\%, 143.

Computational strategies for a 4-component Dirac-Kohn-Sham program.

Leonardo Belpassi, Francesco Tarantelli, Antonio Sgamellotti Dipartimento di Chimica e I.S.T.M.-C.N.R., Università di Perugia, 06123, Italy Harry M. Quiney

School of Physics, The University of Melbourne, Victoria, 3010, Australia

The extension of DFT within the four-component generalization of the KohnSham method, Dirac-Kohn-Sham (DKS) scheme, was formulated by Rajagopal and Macdonald and Vosko. In a previous article, we have described the formulation of a DKS scheme using Gaussian-type basis spinors, the so called $G$-spinors [1]. It was shown that a practical implementation of the DKS scheme may be constructed following closely the lines of the non-relativistic theory. Nevertheless, DKS calculations possess an intrinsically greater computational cost than analogous non-relativistic approaches, mainly because of the preservation of the four-component structure in the representation of the DKS equations. We have recently shown that near-linear scaling of the most computationally demanding parts of the DKS scheme using a $G$-spinor basis may obtained by employing a well-structured parallel processing algorithm [2]. In the present work we extend this parallelization scheme to construct a relativistic implementation of the generalized gradient approximation (GGA) by direct differentiation of the electron density expanded in a $G$-spinor basis. This proves to be a particularly efficient scheme, with an intrinsic computational cost that scales linearly with the number of $G$-spinor basis functions. In order to validate this new implementation a detailed study of the diatomic system $\mathrm{CsAu}$ is also reported. The spectroscopic constants $D_{e}, r_{e}, \omega_{e}$, and $x_{e} \omega_{e}$ and the dipole moment, $\mu$, have been calculated and compared with the best available theoretical and experimental data. The sensitivity of our results to the details of the numerical schemes used to evaluate the matrix elements is analyzed in detail. A comparative study is also presented of molecular properties in the alkali auride series which have been obtained using several standard non-relativistic density functionals.

[1] H. M. Quiney and P. Belanzoni, J. Chem. Phys. 117, 5550 (2002).

[2] L. Belpassi, L. Storchi, F. Tarantelli, A. Sgamellotti, and H. M. Quiney, Future Generation Computer Systems 20, 739 (2004). 
Methodology and Applications

Density functional orbitally resolved reactivity indices in the study of regioselectivity

\section{$\underline{\text { Tzonka Mineva }}$}

Institute of Catalysis, Bulgarian Academy of Science, G. Bonchev Str.11, 1113-Sofia, Bulgaria

Within Density Functional Theory (DFT) were defined rigorously global and local reactivity indices as second order derivatives of the KohnSham (KS) energy to the density, or external potential. In our computational scheme the hardness tensor elements are computed using finite difference derivative of the one-electron Kohn-Sham energies to the change of the occupation number $\left(\Delta n_{i}\right)$ [1]. Further, the softness tensor elements and Fukui indices in orbital resolutions are defined from the orbital hardnesses. A theoretical justification of the relationship between the hardness or softness and chemical reactivity can be given within the hard and soft acids and bases (HSAB) principle of Pearson [2]. The relationship reactivity-softness postulated in HSAB is rationalized in terms of local response properties of the system.

It is well recognized that the accurate prediction of regio- and stereoselectivity requires knowledge about the energetics and the orbital HSAB matching at the assumed reaction sites [3]. We will show several examples of how the orbital reactivity indices can be rationalized within the local HSAB principle to study regioselectivity [4] in the 1,4 addition reaction of lithium derivatives of $\mathrm{N}, \mathrm{N}$-disubstituted phenylacetamides with 2-arylmethylene-1,4-butanolides and in the nucleophylic reaction of 3-( $\omega$-bromoacetyl)coumarin with phosphites.

[1]. N. Neshev and T. Mineva, Metal-Ligand Interactions:Structures and Reactivity, N. Russo and D. R. Salahub (eds), Kluwer, p.361, 1996.

[2]. Pearson, R. G. J. Am. Chem. Soc. 85, 1963, 3533.

[3]. Woodward, S. Tetrahedron 58, 2002, 1017.

[4]. G. Madjarova., A. Tadjer, Tz. P. Cholakova, A. A. Dobrev and T. Mineva, J. Phys. Chem A 109, 2005, 387.

Methodology and Applications

Correlated Optimized Effective Potential Method - orbital dependent exchange-correlation potentials in ab initio Density Functional Theory.

Ireneusz Grabowski $^{1}$, So Hirata ${ }^{2}$, Victor Lotrich ${ }^{2}$, Rodney J. Bartlett ${ }^{2}$

${ }^{1}$ Institute of Physics, Nicolaus Copernicus University, ul. Grudziądzka 5/7, 87-100 Toruń, Poland

2 Quantum Theory Project, University of Florida, Gainesville, USA

From many-body theory and coupled-cluster theory the local exchange-correlation potential of density functional theory in an orbital dependent form is derived. Starting from a general theoretical framework based on the density condition in Kohn-Sham (KS) theory and combining them with Coupled Cluster methodology, we define a rigorous exchangecorrelation functional, potential and orbitals ${ }^{1}$. Specifying initially to second-order terms which defines correlated OEP procedure, we show that our ab initio correlation potential provides the correct shape compared to those from reference quantum Monte Carlo calculations, and we demonstrate the superiority of using Fock matrix elements in defining the unperturbed Hamiltonian. This enables us to introduce $a b$ initio Density Functional Theory as a method that is guaranteed to converge to the right answer in the correlation and basis set limit, just as does $a b$ initio wavefunction theory

A new approximate noniterative procedure ${ }^{2}$ to obtain accurate correlation and exchange-correlation potentials of KS DFT is presented. By carrying out only one step of the correlated OEP, following the standard iterative exchange-only OEP or Hartree-Fock calculations, we can recover accurate correlation potentials that are hardly discernible from those obtained by the more expensive, fully iterative procedure.

[1] R. J. Bartlett , I. Grabowski, S. Hirata, S.Ivanov, J.Chem.Phys. 122 , 034104,(2005)

[2] I. Grabowski, V. Lotrich Mol. Phys. xxxx (2005)

This work has been supported by the Committee for Scientific Research KBN under grant no. 4 T09A 18725.
Methodology and Applications

The role of exact exchange in ab-initio molecular electronics.

\section{Y.García, J.J.Palacios, E.Sanfabián, E.Louis, and J.A.Vergés*}

University of Alicante, San Vicente del Raspeig, Alicante 03690, Spain.

Single atoms/molecules are the next step in reducing active parts of electronic circuits[1]. Nevertheless, an industrial application of these devices requires systematicity at experimental and theoretical levels. From a theoretical point of view, it would be only supported with a description at the ab-initio quantum level.

In this work we show a first principles method developed by our group to study electronic transport intended to provide a powerful tool for dimension-reduced systems[2]. Considering the existence of discrepancies between theoretical groups $[3,4,5]$, presumably working at an ab-initio level, we center the discussion in the Quantum Chemistry Tools in use. The problem derived from the Shrödinger equation for the infinite system must be simplified in order to compute a numerical solution. DFT is currently one of the most useful tools in this respect. The many body problem due to interactions among electrons is typically reduced to local exchange and correlation potentials through DFT. With our results we showed the non-validity of approximations like purely LSDA or GGA, (sometimes the only ones used by physicists), for the selection of the functional. We propose another kind of functionals, more adjusted for atomic/molecular bridged systems, where nonlocality of exchange energy is better taken into consideration.

Finally we present numerical results quantitative supporting previous statements.

[1] R.H.M.Smit et al, Nature, 2002, 419, 906.

[2] J.J.Palacios et al, Phys.Rev.B, 2002, 66, 035322.

[3] Yamila García et al, Phys.Rev.B, 2003, 69, 224425.

[4] K.S.Thygesen et al, arXiv:cond-mat/05011238

[5] Viktor N. Staroverov et al , Journal of Chemical Physics, 2003, 119,12129.

Methodology and Applications

P60

\section{The DFT based chemical reactivity indices in the coupled perturbed ensemble UHF method.}

\author{
Balawender Robert
}

Institute of Physical Chemistry of the Polish Academy of Sciences, Kasprzaka 44/52, PL-01-224 Warsaw, Poland.

A derivation of the density-functional-theory based reactivity indices in the ensemble unrestricted Hartree-Fock (eUHF) method is present. The comparison between the properties of the reactivity indices evaluated within the one- and two-sets of spinorbitals approach of eUHF and hyper UHF method is shown. All approaches give similar Fukui functions irrespectively of used methodology, but the significantly different values for the global indices, which contain the chemical information. Interpretation of the last in the terms of the DFT bases indices can be questionable. The calculational scheme for the indices using the first- and second-order coupled perturbed eHF equations is proposed. A method for identification of the spinorbitals involved in the change of the total number of electron is included. The illustrative examples (water and hydrogen cyanide) show that the ground state (GS) properties of the (electron systems can be predicted from the GS properties of the $Z$-electron systems with accuracy comparable with that of the UHF calculations. The relaxation effect, important for the HCN system in which change in the symmetry of the highest occupied spinorbital occurs, is effectively predicted. 
Methodology and Applications

\section{QED-DFT theory of multi-photon resonances for atoms and molecules in a strong laser field}

\section{$\underline{\text { Alexander V. Glushkov }}$}

Inst. Applied Mathematics, OEU Univ., P.O.Box 24A, Odessa-9, 65009, Atom.-Nucl.-Laser Spectr. Centre, P.O.Box 24A, Odessa-9, 65009, Ukraine

A quantum-electrodynamics (QED) and density functional theory (DFT) method is used for studying interaction of the atoms and molecules with an intense laser field. Method bases on a description of quantum system in a field by the k- photon emission and absorption lines (multi-photon resonances) [1]. The lines are described by their QED moments of different orders, which are calculated with the use of the DFT Dirac-Kohn-Sham scheme and the Gell-Mann \& Low adiabatic formalism and expressed through an imaginary part of energy shift $\operatorname{Im} E_{\alpha}\left(\omega_{0}\right)$ as the function of the laser pulse central frequency. Results of the calculation for the multi-photon resonance and multi-photon ionization profile in the $\mathrm{H}, \mathrm{Na}$, Cs atoms and $\mathrm{H}_{2}$ molecule in the cases of single-, multi-mode, coherent, stochastic laser pulse shape are presented. Our method is compared with other DFT approaches to atoms in a strong laser pulse (c.f.[2]). An approach developed is generalized for a case of additional account for an external DC electric field It is also applied to calculation of probability for two-photon electron detachment from negative atomic ion of $\mathrm{Na}$ (process: $\mathrm{Na}+n \omega \rightarrow \mathrm{Na}+e^{-} ; n=2$ ) by a circularly-polarized light. References:

[1] A.V.Glushkov,JETP Lett.1992, 55,105; A.V.Glushkov,L.N.Ivanov,Phys. Lett.A. 1992, 170, 33; A.V.Glushkov, S.V.Malinovskaya etal, Int.J. Quant. Chem. 2005, to be publ.;

[2] C.A.Ullrich, S.Erhard, E.K.U.Gross, In.: Super Intense Laser Atoms Physics IV, Ed.H.G.Muller, Kluwer, 1996; E.Luc-Koenig,A.Lyras, J-M. Lecomte, M.Aymar, J.Phys.B. 1997, 30, 5213.

[3] A.V.Glushkov, L.N.Ivanov, J.Phys. B. 1993, 26, L379; A.V.Glushkov, S.V.Ambrosov, Int.J.Quant.Chem. 2004, 99, 936; A.V.Glushkov, A.V. Loboda etal, Int.J.Quant.Chem. 2005, to be publ.

Methodology and Applications

New QED approach to construction of the optimized orbital basis's in relativistic density functional theory

\section{Alexander V. Glushkov}

Inst. Applied Mathematics, OEU Univ., P.O.Box 24A, Odessa-9, 65009, Atom.-Nucl.-Laser Spectr. Centre, P.O.Box 24A, Odessa-9, 65009, Ukraine

Within QED theory we develop a new ab initio procedure for construction of the optimized orbital basis's in the relativistic density functional theory (DFT), which refines the Kohn-Sham approach and also the Davidson natural orbitals approach [1]. In the fourth QED perturbation theory there are diagrams, whose contribution into imaginary part of radiation width ImdE of the quantum system accounts for the core polarization multielectron effects [2]. This contribution describes collective correlation effects and it is dependent of the photon propagator gauge (non-invariant ones). A minimization of the functional ImdE leads to the integro-differential DFT like equations, which are solved using the standard atomic code. It allows receiving optimal basis of the relativistic Dirac-Kohn-Sham orbitals. Within the QED it is proved the theorem, generalizing the known Grant theorem [2]. The gauge problem in the relativistic DFT is considered at first on the level of QED and from the point of view of construction of the optimal oneelectron basis's and exactness of calculation results for radiation properties of the atomic systems. One of the examples of checking the efficiency of new procedure is calculation of oscillator strengths for heavy element atoms (some lanthanide's and actinides elements). The calculation results for radiation widths of a number of atoms and ions within traditional approach in the forms of "length" and "velocity" are practically equal for used basis's. References:

[1] A.V.Glushkov,L.N.Ivanov,Phys. Lett.A. 1992, 170, 33; A.V.Glushkov et al, In: New Projects and New lines of research in Nuclear Physics, World Sci. Singapore. 2003, 126, 246; Nucl.Phys.A. 2004, 734, e21; A.V Glushkov, S.V.Malinovskaya etal, Int.J. Quant. Chem. 2004, 99, 889; A.V.Glushkov, A.V. Loboda etal, Int.J.Quant.Chem. 2005, to be publ.; E.Ivanova,L.Ivanov,A.Glushkov, A.Kramida, Phys.Scr. 1985, 32,512;

[2] P.Grant, J.Phys.B. 1974, 71458.
Methodology and Applications

QED-DFT calculation of the hadronic atoms and super heavy ions: energy shifts and widths, decay probabilities

Alexander V. Glushkov, Elena P. Gurnitskaya and Larisa A.Vitavetskaya

Inst. Applied Mathematics, OEU Univ., P.O.Box 24A, Odessa-9, 65009, Atom.-Nucl.-Laser Spectr. Centre, P.O.Box 24A, Odessa-9, 65009, Ukraine E-mail: glushkov@paco.net

A quantum-electrodynamics (QED) and density functional theory (DFT) are applied to calculation of the spectra, energy shifts and widths for and super heavy ions and some hadronic (pion, kaon) atoms. The key problem is establishment of a quantitative link between quality of the nucleus structure modelling and accuracy of calculating energy and spectral properties. A new approach [1] to relativistic calculation of the spectra for multi-electron super heavy ions with an account of relativistic, correlation, nuclear, radiative effects, based on the gauge-invariant QED perturbation theory (PT) is used. The wave functions zeroth basis is found from the Dirac-Kohn-Sham (kaon, atomic ion) or Klein-Gordon (pion atom) equations. The potential includes the effective core potential, electric and polarization potentials of a nucleus. For low orbits there are important effects due to the strong hadron-nuclear interaction (pion atom). For atomic ions the correlation corrections of the high orders are taken into account within the PT with DKS zeroth approximation. The Lamb shift polarization part is accounted in the UhlingSerber approximation, self-energy part of the Lamb shift is accounted for within Green functions method. We present the results of calculation: i). $1 \mathrm{~s}^{3}$ $2 \mathrm{lj}, 3 \mathrm{lj}, 4 \mathrm{lj}$ energy levels, decay probabilities for the Li-like ions; 2). energy spectrum of the atom $Z=118 ; 3$ ). Shifts and widths of some transitions in the pionic and kaonic atoms $\left(\mathrm{K}^{-}{ }^{4} \mathrm{He}\right.$ etc $)$.

References:

[1] A.V.Glushkov,L.N.Ivanov,Phys. Lett.A. 1992, 170, 33; A.V.Glushkov et al, In: New Projects and New lines of research in Nuclear Physics, World Sci. Singapore. 2003, 126; Nucl.Phys.A. 2004, 734, e21; A.V.Glushkov, S.V.Malinovskaya etal, Int.J. Quant. Chem. 2004, 99, 889;

[2] E.Ivanova,L.Ivanov,A.Glushkov, A.Kramida, Phys.Scr. 1985, 32,512; J.Quant.Spectr.Rad.Tr. 1986, 36, 127-145.

Methodology and Applications

Calculation of the alkali diatomics within the DFT perturbation theory method with account for relativistic corrections

\section{Sergey V. Ambrosov}

Inst. Applied Mathematics, OEU Univ., P.O.Box 24A, Odessa-9, 65009, E-mail: glushkov@paco.net

Within formally exact DFT perturbation theory (PT) [1] it is carried out calculation of potential curves for the ground and excited states, spectroscopic properties for the whole number of heavy diatomic alkali dimers $\mathrm{A}_{2}(\mathrm{~A}=\mathrm{Na}, \mathrm{K}, \mathrm{Cs}, \mathrm{Fr})$. The second-and high-order contributions [2] (due to the polarization interaction between valence electrons through polarizable core and mutual screening of above-core valence electrons) are effectively accounted for by using ab initio density functionals. Semiempirical model of the Cohen- Schneider type is used for the treatment of the relativistic (spin-orbit coupling) effects. There are predicted some unusual features in spectra of the heavy alkali diatomics: Rb, Cs, Fr. The effects of giant splitting of the Rb, Cs, Fr dimer $2^{3} \Pi$ state due to the spinorbit interaction have been analyzed. The $2^{3} \Pi$ state is splited into three different states. One is now dealing with three separate electronic transitions rather than only one. For $\mathrm{Fr}_{2}$ dimer the giant splitting effect is first discovered [1]. Under availability of a very narrow line width light source (laser) one may be able to detect these three electronic transitions separately. The constants $\left[\mathrm{T}_{\mathrm{e}}\right.$-excitation energy $\left(100 \mathrm{~cm}^{-1}\right) ; \mathrm{B}_{\mathrm{e}}, \omega_{\mathrm{e}}-$ rotation, vibration constants $\left(\mathrm{cm}^{-1}\right)$ ] for Rydberg state $4 \mathrm{n}^{1} \Sigma_{\mathrm{g}}{ }^{+}$of $\mathrm{Na}$ dimer, calculated by different methods, are as follows: a-experiment [2]; c- empi-rical pseudo potential (PP)+core polarization (CP); b-Hartree-Fock ab initio PP+CP; dthis work (DFT-PT); T=283 (a); 285 (b); 286 (c); 283 (d); B= 0,0899 (a); 0,0838 (b); 0,088 (c); 0,0895 (d); $\omega=108,7$ (a); 107 (b); 105 (c); 108,1 (d). References:

[1] A.V.Glushkov, S.V.Ambrosov, et al, J.Struct.Chem. 1999, 39, 621; In: New Projects and New lines of research in Nuclear Physics, World Sci. Singapore. 2003, 126; Nucl.Phys.A. 2004, 734, e21; A.V.Glushkov, S.V.Malinovskaya etal, Int.J. Quant. Chem. 2005, to be publ.; [2] A.Henriet, F.Masnou-Seeuws, J.Phys.B: 2988, 21, L339-348. 
Methodology and Applications

Monte-Carlo and DFT computational simulation of energy and structural properties of the prostaglandin biomolecules in solution

\section{$\underline{\text { Andrey V.Malinovsky }}$}

Odessa Medicine University, P.O.Box 116, Odessa-9, 65009, Ukraine E-mail: glushkov@paco.net

In last years a studying the structural, energy and photo-physical properties for such biological objects as enzymes etc [1] is of a great interest. Here we present the results of the Monte-Carlo (MC)\& DFT computational studying the energy and structural characteristics of the prostaglandins PG (also prostacycliens, thromboxans) biomolecules in the water solution (cluster). A cluster, consisting of the PG and hundred water molecules, is studied. All relevant interaction potentials had been obtained by means of the DFT calculations [2,3]. The molecule geometry's for the solute together with additional data (computed net charge and «class» for each atom etc.) are taken and compilated from [1-3]. Calculations were carried out at $\mathrm{T}=300 \mathrm{~K}$ and all molecules were treated as rigid. Results for potential energies are following (in $\mathrm{kJ} \mathrm{mol}^{-1}$ ): Water-water (neutral molecule) $-28.8 \pm 0.5$ and («zwitterion») -28.5 \pm 0.5; PG-water (neutral molecule) $-63.6 \pm 2.5$ and («zwitterion») $-375.6 \pm 15.0$; The MC result for bulk water (with the same interaction potential) is $35,6 \pm 0.6 \mathrm{~kJ} \mathrm{~mol}^{-1}$ [2]. The structural characteristics (radial distribution and orientational correlation functions) are also analyzed. The «zwitterion» appears is to be strongly favored with respect to the neutral molecule. In this situation one can wait for the PG phenomenal biocalalytic and photochemical activity. The similar data are obtained for other biomolecules, namely, also prostacycliens and thromboxans.

References

[1] V.Schreiber, Pathophysiology of Endocrine Glands, Avic., Praga, 1987.

[2] G.Ranghino, S.Romano, E.Clementi, Gazz. Chim. Ital. 1978, 108, 157.

[3] A.Glushkov, Journ.Struct.Chem. 1993, 34, 3; 1990, 31, 9; Sov. Journ.

Phys. Chem. 1991, 65, 2970; 1992, 66, 1259; A.Glushkov , L,Ivanov, J.Quant.Spectr. Rad. Trans. 1986, 36,127; Phys.Lett.A. 1992, 170, 33; A.Glushkov, A.Malinovsky et al, Journ.Struct.Chem. 1998, 39, 21.

Methodology and Applications

Quantum Electrodynamics DFT calculation of the atomic parity nonconservation effect and the beta decay probabilities

Svetlana V. Malinovskaya

Odessa University, P.O.Box 24A, 65009, Odessa, Ukraine

A consistent QED-DFT (operator QED perturbation theory with DFT zeroth approximation) method [1] is used in calculating beta decay characteristics and atomic parity non-conservation effect. As method of calculation of the atomic fields and wave functions we used the gauge invariant scheme of the Dirac-Kohn-Sham approach [1]. The Fermi model and gauss form of charge distribution in a nucleus are used. Approach allows to calculate continuum wave functions with full account of exchange of the considered continuum electron with electrons of the atom. Two schemes of calculation are used: i). relativistic electron radial wave functions are calculated on the boundary of spherical nucleus; ii). values of these functions in the zero are used. We have considered the $\beta$ decays: ${ }^{33} \mathrm{P} \rightarrow 6{ }^{33} \mathrm{~S},{ }^{35} \mathrm{~S} \rightarrow{ }^{35} \mathrm{Cl},{ }^{45} \mathrm{Ca} \rightarrow{ }^{45} \mathrm{Sc},{ }^{63} \mathrm{Ni} \rightarrow{ }^{63} \mathrm{Cu}$, ${ }^{106} \mathrm{Ru} \rightarrow{ }^{106} \mathrm{Rh},{ }^{155} \mathrm{Eu} \rightarrow{ }^{155} \mathrm{Gd},{ }^{241} \mathrm{Pu} \rightarrow{ }^{241} \mathrm{Am}$. We also carried out calculation of energy levels, E1,M1-transitions amplitudes in heavy atoms of $\mathrm{Cs}, \mathrm{Pb}$. Let us present the results for parity non-conserving $6 \mathrm{~s}-7 \mathrm{p}$ dipole amplitude in Cs. Result of our calculation: $\mathrm{D}=<6 \mathrm{~s}|\mathrm{Dz}| 7 \mathrm{~s}\rangle=-0.92 \mathrm{~d}, \mathrm{~d}=10^{-11} \mathrm{ilela}(-\mathrm{Qw} / \mathrm{N})$. For comparison let us present the data: $\mathrm{D}=-0.91 \mathrm{~d}$ by Dzuba etal; $\mathrm{D}=-0.935 \mathrm{~d}$ by Bouchiat etal (Paris); $\mathrm{D}=-0.935 \mathrm{~d}$ by Johnson etal (Indiana) [2]. The comparison of calculated D value with measurement of Noeker et al gives the values of weak nuclear charge in Cs and Wainberg angle: $(-\mathrm{Qw} / \mathrm{N})=$ $0,918 \pm 0,020$ (exp) $\pm 0,010$ (th); $\sin ^{2} \vartheta_{w}=0,231 \pm 0,007$ (exp) $\pm 0,004$ (th) that is in a good agreement with average world value $\sin ^{2} \vartheta_{w}=0,230 \pm 0,005$.

References

[1] A.Glushkov et al, In: New Projects and New lines of research in Nuclear Phys.,eds.Fazio G.,Hanappe F.-World Sci. Singapore. 2003,126; Nucl.Phys A. 2004, 734, e21; S.V.Malinovskaya, Int.J. Quant. Chem. 2005, to be publ. [2] I.Band etal, Izv. AN USSR. 1987, 51, 1998; N.Noeker etal, Phys.Rev.A. 1988, 37, 1395; W.Johnson etal, Phys.Scr.1993, 46 184; C.Bouchiat, C. Piketty, Europh. Lett.1986, 2,511; V.Dzuba et al,Phys.Rev.A 1991,44, 828;
Methodology and Applications

\section{QED-DFT theory of laser-electron-nuclear processes in the molecular systems}

Svetlana V.Malinovskaya

Odessa University, P.O.Box 24A, Odessa-9, 65009, Ukraine E-mail: glushkov@paco.net

A new class of problems has been arisen in quantum physics and quantum chemistry and connected with modelling co-operative laser-electron-nuclear phenomena in molecules. It includes a calculation of the probabilities and energies of the mixed $\gamma$-optical quantum transitions in atomic and molecular systems, intensities of the complicated $\gamma$-transitions due to the changing of the atomic or molecular excited state population under action of laser radiation, quantum chemical calculation of the complex "laser-electronnuclear" system. A new QED-DFT approach (c.f.[1-3]) to calculation of the electron-nuclear $\gamma$ transition spectra (set of vibration-rotational satellites in molecule) of nucleus in molecule is proposed. Within it a probability of decay of the excited state is defined as an imaginary part of the energy shift for the nucleons- electron shells-electromagnetic field system. Estimates are made for vibration-rotation-nuclear transition probabilities in a case of the emission and absorption spectrum of nucleus ${ }^{127} \mathrm{I}\left(\mathrm{E}^{(0)}{ }_{\gamma}=203 \mathrm{keV}\right)$, in the molecule $\mathrm{H}^{127} \mathrm{I}$. There is discussed a dependence of calculation results upon form of the density functional. The possible generalization of the theory for molecules of the following type: three-atomic $X_{2}\left(D_{\propto h}\right)$, four-atomic $X Y_{3}$ $\left(D_{3 h}\right)$, five-atomic $X_{4}\left(T_{d}\right)$, six-atomic $X_{3} Y_{2}\left(D_{3 h}\right)$, seven-atomic $X Y_{6}$ $\left(\mathrm{O}_{\mathrm{h}}\right)$ is also considered.

References:

[1] L.N.Ivanov,V.S. Letokhov, JETP.1987, 93, 396; A.V.Glushkov, L.N. Ivanov, Phys.Lett.A. 1992, 170, 33.

[2] A.V.Glushkov, S.V.Malinovskaya, Int.J.Quant.Chem. 2004, 99, 889; In: New Projects and New lines of research in Nuclear Physics, eds.Fazio G. And Hanappe F.-World Sci. Singapore. 2003, 242.

[3] S.V.Malinovskaya, Int.J.Quant.Chem. 2005, to be publ.

Methodology and Applications

DFT description of van der Waals forces with explicit long-range interactions

Iann C. Gerber, János G. Ángyán

$\mathrm{LCM}^{3} \mathrm{~B}$, UMR 7036, Université Henri Poincaré, Vandœuvre-lès-Nancy, France

Dispersion forces (van der Waals) are long-range electronic correlation effects, which are missed almost entirely by usual local or semi-local functionals approaches. The most important manifestation of this misbehaviour is the incorrect asymptotical decay of the DFT interaction energy between closed-shell systems. Furthermore, it must be noted that van der Waals minimum region is described in an erratic manner by DFT, LDA tends to overbind with a too small bond-length, few gradient corrected functionals do not bind while others give a minimum usually too small. After a separation of the electron-electron interaction [1] in the Hamiltonian into short- and long-range components, long-range exchange and correlation effects, which are responsible for the failure of currently used approximations, are taken into account explicitly. We propose a "range separated hybrid" functional based on a local density approximation for the short-range exchange-correlation energy [2], combined with long-range exact exchange energy. Furthermore, long-range correlation effects are added by a second-order pertubational treatment which avoids the double-counting of correlation and gives the correct asymptotical behaviour with a good description of the minimum region. Test cases like rare gas dimers closed shell systems prove the accuracy of the scheme and allow for an investigation of more complex systems [3].

[1] A. Savin, On degeneracy, near-degeneracy and density functional theory, in Recent developments ans Applications of Modern Density Functional Theory, edited by J. M. Seminario, page 327, Elsevier, Amsterdam, 1996.

[2] J. Toulouse, F. Colonna, and A. Savin, Phys. Rev. A, 2004, 70, 062505 .

[3] J. Ángyán, I. Gerber, A. Savin and J. Toulouse, cond-mat, 2005, 0505062 
Methodology and Applications

Theoretical aspects of the electronic affinities calculations for molecule in quartet ground electronic state

\section{Valentina Shvets}

University "Ukraine”, Ukraine, 04071, Kyiv, Choriva-Street, 1-G

The present report represents the theoretical aspects of the electronic affinities calculations for molecule in quartet ground electronic state using the "sum method" by Ziegler and Rauk [1]. For writing of the excited configuration $a^{1} b^{1} c^{1} d^{1}$ have been used 16 determinants, corresponding $M_{s}=0,1,-1,2,-2$.

The results of operation $\hat{S}^{2}=\widehat{S}_{-} \widehat{S}_{+}+\widehat{S}_{z}^{2}+\widehat{S}_{z}$ on each from determinants have been represented. The 16 wave functions of singlets, triplets and quintet states have been received using the equation: $\hat{S}^{2} \psi=S(S+1) \psi$.In according with the "sum method" have been received the expression for $E\left(D_{i}\right)$, for example:

$$
\begin{aligned}
& E\left(D_{1}\right)=\frac{1}{6} E_{\text {quin }}+\frac{1}{12} E_{S 2}+\frac{1}{4} E_{S 1}+\frac{1}{2} E_{T 3}, E\left(D_{2}\right)=\frac{1}{6} E_{\text {quin }}+\frac{1}{12} E_{S 2}+\frac{1}{4} E_{S 1}+\frac{1}{2} E_{T 2} \\
& E\left(D_{3}\right)=\frac{1}{6} E_{\text {quin }}+\frac{1}{2} E_{S 2}+\frac{1}{2} E_{T 2}, E\left(D_{7}\right)=\frac{1}{4} E_{\text {quin }}+\frac{1}{4} E_{T 3}+\frac{1}{2} E_{T 1} \\
& E\left(D_{10}\right)=\frac{1}{4} E_{\text {quin }}+\frac{1}{4} E_{T 3}+\frac{1}{2} E_{T 2}, \\
& \text { where } D_{1}=\left|a^{+} b^{+} c^{-} d^{-}\right|, D_{2}=\left|a^{+} b^{-} c^{+} d^{-}\right|, D_{3}=\left|a^{+} b^{-} c^{-} d^{-}\right|, D_{7}=\left|a^{+} b^{+} c^{+} d^{-}\right| \\
& D_{10}=\left|a^{+} b^{+} c^{-} d^{+}\right| .
\end{aligned}
$$

The expressions for electronic affinity energies using the expressions for $E\left(D_{i}\right)$ are received.

[1] T. Ziegler, A. Rauk, E. J. Baerends, Theor.Chim.Acta, 1977, 43(3),261.

Methodology and Applications

\section{Relaxation dynamics and structural isomerism in $\mathbf{N b}_{10}$}

\section{T. R. Walsh}

Dept. of Chemistry and Centre for Scientific Computing, University of Warwick

An explanation for the observed biexponential reaction kinetics of $\mathrm{Nb}_{10}[1-$ 3 with respect to small molecule adsorbates is sought in this theoretical study. The structure, energetics and interconversion of isomers of $\mathrm{Nb}_{10}$ are determined using density functional theory with Gaussian basis sets, using guess structures derived from basin-hopping simulations [4], with the Finnis-Sinclair potential[5]. Ionization potentials are calculated for all relevant minima. Using these interconversion data, the master equation approach is applied to study relaxation dynamics of $\mathrm{Nb}_{1 \mathrm{c}}$ via the diamond-square-diamond rearrangement mechanism.

[1] M. B. Knickelbein and S. H. Yang, J. Chєm. Phys., 1990, 93, 1476.

[2] M. B. Knickelbein and S. H. Yang, J. Chєm. Phys., 1990, 93, 5760.

[3] C. Berg, T. Schindler, M. Kantlehner, G. Niedner-Scatteburg, and V. E. Bondybey, Chem. Phys., 2000, 262, 143.

[4] D. J. Wales and J. P. K. Doye, J. Phys. Chem. A, 1997, 101 5111.

[5] M. W. Finnis and J. E. Sinclair, Phil. Mag. A, 1984, 50, 45.
Methodology and Applications

Weak interactions studied with Hartree-Fock exchange + Wilson-Levy correlation

\section{T. R. Walsh}

Dept. of Chemistry and Centre for Scientific Computing, University of Warwick

The Wilson-Levy (WL) correlation functional is used together with Hartref Fock theory to evaluate interaction energies at intermediate separations (i.e. around equilibrium separation) for several weakly-bonded systems. The HF +WL approach reproduces binding trends for all complexes studied: selected rare-gas dimers, isomers of the methane dimer, benzene dimer and naphthalene dimer, and base-pair stacking structures for pyrimidine, cytosine, uracil and guanine dimers. These HF+WL data are contrasted against results obtained from some popular functionals (including B3LYP and PBE), as well as two newly-developed functionals, $\mathrm{X} 3 \mathrm{LYP}[1]$ and $\mathrm{xPBE}[2]$. The utility of $\mathrm{HF}+\mathrm{WL}$, with reference to exactexchange[3-6] (EXX) density-functional theory, is discussed in terms of a suggested EXXWL exchange-correlation functional.

[1] X. Xu and W. A. Goddard, J. Chem. Phys., 2004, 121, 4068.

[2] X. Xu and W. A. Goddard, Proc. Nat. Acad. Sci., 2004, 101. 2673.

[3] R. T. Sharp and G. K. Horton, Phys. Rev., 1953, 90, 317.

[4] J. D. Talman and W. F. Shadwick, Phys. Rev. A., 1976, 14 36

[5] F. DellaSalla and A. Görling, J. Chem. Phys., 2001, 115 5718.

[6] W. T. Yang and Q. Wu, Phys. Rev. Lett., 2002, 89, 143002.

Methodology and Applications

Implementation of hybrid functionals into WIEN2k

F. Tran ${ }^{1}$, P. Novák ${ }^{2}$, P. Blaha ${ }^{1}$, and K. Schwarz ${ }^{1}$

${ }^{1}$ Institute for Materials Chemistry, Vienna University of Technology, Getreidemarkt 9/165-TC, A-1060 Vienna, Austria

${ }^{2}$ Institute of Physics, Academy of Sciences of the Czech Republic, Cukrovarnická 10, CZ-162 53 Prague 6, Czech Republic

For the treatment of localized electrons of selected atom and orbital momentum we propose to subtract the corresponding approximate LDA or GGA functional for the exchange energy and to add the exact HartreeFock exchange instead [1]. This constitutes a simplified version of the usual implementation of the Hartree-Fock formalism. Starting from this, we construct exchange-correlation functionals of the hybrid form like B3PW91, PBE0, etc. These functionals are tested on several transition metals compounds and the results are compared with the LDA, GGA, $\mathrm{LDA}+U$, and experimental ones. For instance, for $\mathrm{NiO}$ there is a good agreement between our results and the experimental ones for the gap, equilibrium volume, and magnetic moment

[1] P. Novák, J. Kuneš, W.E. Pickett, L. Chaput (manuscript in preparation) 
Methodology and Applications

\section{Chemical Reactivity and the Shape Function}

$$
\text { P. Geerlings*, F. De Proft*, P.W. Ayers\# }
$$

*Eenheid Algemene Chemie, Faculty of Sciences Free University of Brussels, Pleinlaan 2, 1050 Brussels, Belgium \#Department of Chemistry, McMaster University Ontario, Canada, L8S4M1

The role of the shape function $\sigma(\underline{r})$ [1] as an alternative to the electron density function $\rho(\underline{r})$ as an even simpler carrier of information [2] is investigated. Recent developments involving the extension of Mezey's holographic theorem to the shape function [3], the shape function as a primer for reactivity descriptors and some pragmatic approaches to extract $\mu, \eta, f(\underline{r}), s(\underline{r}) \ldots$. from the shape function are discussed [4,5]. Finally the decisive role of the shape function in atomic and molecular similarity analyses is discussed.

[1] R.G. Parr and J. Bartolotti, J. Phys. Chem., 87, 2810 (1983)

[2] P.W. Ayers, Proc. Natl. Acad. Sci. (USA), 97, 1959 (2000)

[3] P. Geerlings, G. Boon, C. Van Alsenoy, F. De Proft, Int. J. Quant. Chem., 101, 722 (2005)

[4] F. De Proft, P.W. Ayers, K.D. Sen, P. Geerlings, J. Chem. Phys., 120, 9969 (2004)

[5] P. Geerlings, F. De Proft, P.W. Ayers, Theoretical Aspects of Chemical Reactivity, A. Toro-Labbé, Editor, Theoretical and Computational Chemistry, Vol. 16, P. Politzer Editor, Elsevier, submitted.

Methodology and Applications

\section{Electronic structure studies of some Cobalt complexes using TD-DFT methods}

$$
\text { Prabha Jayapal, Mark A Vincent, Ian H Hillier }
$$

School of Chemistry, University of Manchester, Manchester- M13 9PL.UK

$$
\text { Prabha.jayapal@stud.man.ac.uk }
$$

Time-dependent density functional theory (TD-DFT) has proved to be a useful method for calculating the excitation energies for inorganic complexes, and can be used to interpret the photoelectron spectra of these molecules. A series of $\left(\eta^{5}\right.$ cyclopentadienyl)-ene-1,2-dithiolato cobalt complexes have been studied by TD-DFT methods to determine their ionization energies and thus aid the interpretation of their photoelectron spectra.
Methodology and Applications

\section{Computational studies on redox properties of Rubredoxin mutants-} A DFT and ONIOM study

Mahesh Sundararajan, Jonathan P McNamara and Ian H Hillier.

School of Chemistry, University of Manchester, Manchester - M13 9PL. UK

$$
\text { mahesh.sundararajan@stud.man.ac.uk }
$$

Iron-Sulfur proteins are ubiquitous in living organisms which function as potential electron carriers. Rubredoxins are a class of iron sulfur proteins whose active site contains a high spin iron atom tetrahedrally coordinated by four cysteine sulfurs. The redox potentials of Rubredoxins vary from $-100 \mathrm{mV}$ to $+100 \mathrm{mV}$. The redox potentials are decreased to $200 \mathrm{mV}$ compared to the native proteins when one active site cysteine is replaced by serine. We used DFT and ONIOM methods (with electronic and mechanical embedding) to study the origin of this decrease in redox potentials.

\section{Structure and Reactivity of Aromatic All-Metal Clusters: A DFT Theoretical Study.}

$\underline{\text { Juvencio Robles, Gabriel Merino and Paulina González }}$

Facultad de Química, University of Guanajuato, Noria Alta s/n, Guanajuato, Gto. 36050, México. Phone/Fax: +52 473 732-0006 ext.8120,roblesj@quijote.ugto.mx

It has been argued that some small newly discovered all-metal clusters may be considered as aromatic. This would make them the first aromatic chemical systems with no-carbon content at all ${ }^{[1],[2]}$

The initial report was on the ionic aluminum cluster, $\mathrm{Al}_{4}^{-2}$, known to exhibit a plane-square structure and possess two pi-delocalized electrons, thus satisfying Huckel's rule for aromatic systems. It also shows a relative high chemical and structural stability. Kuznetsov et al ${ }^{2}$ through theoretical calculations have shown that this basic aluminum square structure is preserved when the $\mathrm{Al}$ cluster reacts to form some bi-metallic systems with chemical compositions: $\mathrm{MAl}_{4}{ }^{-}$and $\mathrm{M}_{2} \mathrm{Al}_{4}$ (where $\mathrm{M}=\mathrm{Li}^{+}, \mathrm{Na}^{+}$and $\mathrm{Cu}^{+}$) and aromatic nature is apparently preserved.

In this paper we report our density functional theory (DFT) study and calculations at the B3LYP/6-31+G(d) level of theory/numerical precision which allowed us to rationalize and quantify the aromatic nature of the above mentioned $\mathrm{MAl}_{4}^{-}$species and thereafter to extend our study to other bi-metallic systems that had not been previously reported with general composition $\mathrm{MAl}_{4}{ }^{\mathrm{n}}\left(\mathrm{M}=\mathrm{Li}^{+}, \mathrm{Na}^{+}, \mathrm{K}^{+}, \mathrm{Be}^{2+}, \mathrm{Mg}^{2+}, \mathrm{Ca}^{2+}, \mathrm{Sc}^{3+}, \mathrm{Al}^{3+}, \mathrm{B}^{3+}, \mathrm{Ga}^{3+}\right.$, $\mathrm{Ti}^{4+}$ ) where $\mathrm{n}=-1,0,+1$. We also assess the cluster stability as a function of charge and discuss different manners to assess the aromaticity of these systems, namely through reactivity indices such as the absolute hardness ${ }^{[3]}$, NICS, DI, molecular electrostatic potentials, HOMO and LUMO wave functions and energies. The usefulness of these DFT-calculated parameters to assess and quantify the aromaticity in these novel clusters is discussed.

[1] Li X.; Pennington W.; Robinson G. J Am. Chem. Soc., 117, 7578-7579 (1995).

[2] Li Xi, Kuznetsov A. E., Zhang H, Boldyrev A. I., Wang LS. Science, 291, 859-861 (2001)

[3] Zhou Z. y Navangul H. V. Journal of Physical Organic Chemistry, 3, 784-788 (1990) 
Methodology and Applications

Application of DFT to analysis of the hyperfine parameters and the bonding in donor-acceptor complexes O. Kh. Poleshchuk ${ }^{1}$, V. Branchadell ${ }^{2}$, A. V. Fateev ${ }^{1}$, A. Schulz $^{3}$, A. Scagnelli ${ }^{4}$, B. Brycki ${ }^{5}$

${ }^{1}$ Tomsk State Pedagogical University, Tomsk, Russia

${ }^{2}$ Universitat Autonoma de Barcelona, Barcelona, Spain

${ }^{3}$ Ludwig-Maximilians-Universität, München, Germany.

${ }^{4}$ Universita di Milano-Bicocca, Milano, Italy

${ }^{5}$ A. Mickiewicz University, Poznan, Poland

Structure and bonding in Lewis acid-base complexes has long been a subject of much chemical interest. Central to most simple theories of Lewis acidity is the idea that both ionic and covalent interactions play important roles in stabilizing the donoracceptor bond. In this report, we present a DFT calculations based on a microwave spectroscopic, nuclear quadrupole resonance and Mössbauer investigation of the donor-acceptor complexes formed from metal halogenides with organic ligands. We examinee a broader series of acceptors adducts, with focus on both hyperfine parameters and computationally derived binding energies. The full optimisations of geometry were carried out using such functionals as B3LYP, BHandHLYP, B3PW91 achieved within the GAUSSIAN'98 program. The formation energy of the complexes has been broken down using the extended transition state scheme implemented in the ADF'2004 package. Our calculations showed that in energy of the complex formation of the different complexes depends differently from the covalent and ionic interactions. The use of the energy partitioning scheme has enabled receive the answer to a question about the relative contributions of covalent and electrostatic interactions to the donoracceptor bond.

We thank the Cariplo Foundation organized by the Landau Network-Centro Volta for providing a research grant.

Methodology and Applications

Assessment of several hybrid density functionals for the evaluation of the bond length alternation of increasingly long oligomers

Denis Jacquemin $^{1}$, Eric. A. Perpète ${ }^{1}$, Jean-Marie André ${ }^{1}$, Henry Chermette $^{2}$, I. Ciofini ${ }^{3}$ and C. Adamo ${ }^{3}$

(1) Laboratoire de Chimie Théorique Appliquée, Facultés Universitaires Notre-Dame de la Paix, rue de Bruxelles, 61, B-5000 Namur, Belgium.

(2) Laboratoire de Chimie Physique Théorique, Université Claude Bernard, Bat. 210, Lyon I and CNRS UMR 5182, 43, Boulevard du 11 Novembre 1918, F-69622 Villeurbanne Cedex, France.

(3) Laboratoire d'Electrochimie et Chimie Analytique, UMR CNRSENSCP ${ }^{\circ} 7575$, Ecole Nationale Supérieure de Chimie de Paris, , 11 rue P. et M. Curie, F-75231 Paris Cedex 05, France.

The bond length alternation (BLA) of increasingly long polyacetylene and polymethineimine oligomers has been computed using wavefunction methods of increasing accuracy and several DFT models, including standard GGA and most-recent meta-GGA and hybrid functionals [1-3]. Our results show that the meta-GGA do not offer any significant improvement with respect to the GGA [2,3], both providing too small BLA values. For polyacetylene, the most accurate results are obtained with hybrid approaches, in particular hybrids including $25 \%$ of HF exchange [2]. On the contrary for polymethineimine, the results of hybrid-DFT geometries are in poor agreement with MP2 geometries [3].

[1] D. Jacquemin, A. Femenias, H Chermette, J.M. André, and E. A. Perpète, in preparation

[2] D. Jacquemin, E. A. Perpète, I. Ciofini, and C. Adamo, Chem. Phys. Lett., accepted

[3] D. Jacquemin, J.M. André and E. A. Perpète, J. Chem. Phys., 2004, 121, 4389.
Methodology and Applications

The study of electronic structure and reactivity of iodinating agents by ab initio and the DFT methods

\author{
E. A. Krasnokutskaya ${ }^{1}$, V. D. Filimonov ${ }^{1}$, O. Kh. Poleshchuk $^{2}$ \\ ${ }^{1}$ Tomsk Polytechnic University, Tomsk, Russia \\ ${ }^{2}$ Tomsk State Pedagogical University, Tomsk, Russia
}

One of the most widely used iodizing agents is some compounds with iodine-heteroatom bonds I-X. The general property for these compounds is the fact that the iodine is bonded with more electronegative atom $(\mathrm{X}=\mathrm{Cl}, \mathrm{N}, \mathrm{O})$ and therefore polarization of the bonds ensures deficit of the electronic density on the iodine and increases its electrophilic character.

Quantum-chemical calculations by MP2/LanL2DZ and $\mathrm{B} 3 \mathrm{LYP} / 6-311 \mathrm{G}^{*}$ have determined electronic and spatial structure of the wide range of the compounds with the $\mathrm{X}-\mathrm{Hlg}(\mathrm{X}=\mathrm{N}, \mathrm{O}, \mathrm{Cl}$; $\mathrm{Hlg}=\mathrm{Cl}, \mathrm{Br}, \mathrm{I})$ bonds in the neutral and protonated forms and also different electronic forms of the $\mathrm{I}_{3}{ }^{+}$cation. We have been used the Becke3LYP exchange-correlation functional in conjunction with small-core relativistic effective core potential for iodine atom. Calculations were performed both for a gas phase and the solutions. Thermodynamic parameters of the interaction between the compounds R-X-I and hydroxyl-containing solvents $(\mathrm{ROH})$ were calculated using the PCM model.

As a result of the theoretical analysis, the quantitative dependences between the structure of neutral and protonated forms of the hypoiodite character R-X-I and indexes of their electrophilic reactivity were revealed for the first time.

Methodology and Applications

Adsorption of $\mathrm{S}$ on (001), (110) and (111) surfaces of $\mathrm{CeO}_{2}-$ Performances of various hamiltonians

$\underline{\text { Ph. Baranek }}{ }^{1}$, L. Gautier ${ }^{2}$ and M. Marrony ${ }^{2}$

EDF R\&D - MMC, BP. 46., Avenue des Renardières, 77818 Moret-sur-Loing Cedex, France

European Institute for Energy Research, Emmy-Noether Strasse 11, 76131 Karlsruhe, Germany

Sulphur containing molecules $\left(\mathrm{H}_{2} \mathrm{~S}\right.$, mercaptans,...) are common compounds in natural gas and liquid fuels (gasoline) or added as odorants for safety reasons These impurities are responsible for the poisoning of catalysts used in many chemical processes such as fuel processing for hydrogen production and for fue cells [1]. They cause either deactivation or obstruction of catalytic sites. Specific units are therefore implemented at the beginning of the process in order to remove sulphur containing molecules bringing complexity and cost to the overall system. An other option which would constitute a breakthrough in this field would be to develop sulphur tolerant catalysts. Ceria $\left(\mathrm{CeO}_{2}\right)$ is an important ceramic material exploited in a wide range of applications such as solid oxide fuel cells. A specific ceramic oxide, the gadolinium doped ceria $\left(\mathrm{Ce}_{0.8} \mathrm{Gd}_{0.2} \mathrm{O}_{1.9}\right)$ has been recognised as a sulphur tolerant material [2]. Its tolerance towards a blended gasoline (with benzothiophene up to $50 \mathrm{ppm}$ sulphur concentration) was proved over $1700 \mathrm{~h}$ of operation. Then it is important to understand its surface catalytic properties. The adsorption of $\mathrm{S}$ on the (001), (110) and (111) surfaces of ceria is investigated at the $a b$ initio quantum mechanical level, by using the periodic CRYSTAL03 and PWSCF codes. The equilibrium lattice parameters of $\mathrm{CeO}_{2}$, surface stabilities, lattice formation and $\mathrm{S}$ adsorption energies have been evaluated. The calculations have been performed at the Hartree-Fock (HF), density functional theory (DFT) and hybrid levels. A good agreement between calculated and experimental various properties has been found with hybrid approximations. The influence of $\mathrm{Ce} f$ orbitals on these various properties is commented.

[1] M. Krumpelt, T.R. Krause, J.D. Carter, J.P. Kopasz and S. Ahmed, Catalysis Today 2002, 77, 3 .

[2] M. Krumpelt et al., Patent US2002/0193247A1, “Authermal hydrodesulfuring reforming catalyst" 
Methodology and Applications

First-principles electronic structure of $\mathrm{Ni}_{2} \mathrm{MnGe:}$ a possible band gap.

\section{Pugaczowa-Michalska}

Institute of Molecular Physics, Polish Academy of Science, Smoluchowskiego 17, 60-179 Poznań, Poland

First principle calculations predicted some Heusler alloys to be halfmetallic $[1,2]$. Half-metallic compounds have electronic states of only one spin direction at the Fermi level. However, there is a gap in the density of states (DOS) for the other spin direction at the Fermi level. A possibility of the band gap for one of the spin directions in the electronic structure of $\mathrm{Ni}_{2} \mathrm{MnGe}$ (in the $\mathrm{L} 2_{1}$ structure) has studied by first principles methods: tigh-binding and full-potential linear-muffin-tin-orbital (TB-LMTO and LmtART) [3,4]. Exchange-correlation potential is used in the local-spin-density approximation (LSDA). Results of our calculation show that $\mathrm{Ni}_{2} \mathrm{MnGe}$ is ferromagnetic ordered at $0 \mathrm{~K}$. In the present system, the band gap near the Fermi level is possible to occur for the minority spin direction. We observe separation of bands near the Fermi energy due to the tetragonal distortion as well as the change of atomic positions between $\mathrm{Ni}$ and $\mathrm{Mn}$ atoms. The effect of a lattice parameter on a size of the band gap is also considered.

[1] R. A. De Groot, F. M. Mueller, P. G. Van Engen, K. H. Buschow, Phys. Rev. Lett., 1990, 50, 2024.

[2] S. Fujii, S. Sugimura, S. Ishida, S. Asano, J. Phys.: Cond Matter, 1990, 43, 8583.

[3] O. K. Andersen, Phys. Rev. B, 1975, 13, 3050.

[4] S. Y. Savrasov, Phys. Rev. B, 1996, 54, 16470.

Methodology and Applications

Volume effect on the magnetic properties of $\mathrm{Fe}_{3-\mathrm{x}} \mathrm{Cr}_{\mathrm{x}} \mathrm{Al}$

$\underline{\text { Anna Go }}{ }^{1}$, Maria Pugaczowa-Michalska ${ }^{2}$, Ludwik Dobrzyński ${ }^{1,3}$

Institute of Experimental Physics ${ }^{1}$

University of Białystok, Lipowa 41, 15-424 Białystok, Poland Institute of Molecular Physics ${ }^{2}$

Polish Academy of Science, Smoluchowskiego 17, 60-179 Poznań, Poland The Soltan Institute for Nuclear Studies ${ }^{3}$ 05-400 Otwock-Świerk, Poland

$\mathrm{Fe}_{3-\mathrm{x}} \mathrm{Cr}_{\mathrm{x}} \mathrm{Al}$ alloys, in the range $0 \leq \mathrm{x} \leq 0.5$, crystallise in the $\mathrm{DO}_{3}$-type structure [1]. The decrease of the magnetic moment of iron with the increase of the chromium concentration was obtained by Mössbauer measurements [2]. However, detailed theoretical investigations [3] for the experimental values of the lattice constant [2] show different gradient of the total magnetic moment versus lattice constant. Furthermore there is quite unexpected behaviour of magnetic moment of iron atoms on one of the non-equivalent positions, namely, the magnetic moment increases with the concentration of chromium. It is well known that magnetic moment of pure iron depends strongly on the Wigner-Seitz radii [4]. The purpose of this work is to investigate the dependence of the total and local magnetic moments on the lattice constant. The electronic structure for ordered $\mathrm{Fe}_{3-\mathrm{x}} \mathrm{Cr}_{\mathrm{x}} \mathrm{Al}$ alloys have been obtained using the self-consistent spin-polarised TB-LMTO method [5]. Calculations were carried out for the changing values of the lattice constant in range $\pm 15 \%$ in relation to the experimental values [2]. Obtained results show that the values of magnetic moments would agree with the experiment if the change of the lattice parameter was about $5 \%$

[1] D. Satuła, et at., J. Magn. Magn. Mater., 1995, 140-144, 61.

[2] D. Satuła, et al., J. Magn. Magn. Mater., 1997, 169, 240.

[3] A. Go, et al., Molecular Physics Reports, 2003, 38, 86.

[4] D. Bagayoko, J. Callaway, Phys. Rev. B, 1983, 28, 5419.

[5] O. K. Andersen, O. Jepsen, Phys. Rev. Lett., 1984, 53, 2571.
Methodology and Applications

P82

Theoretical Studies on the Electronic Properties of Transition Metal Complexes using DFT and Ligand Field Theory

\section{M.Atanasov ${ }^{\mathrm{ab}, \mathrm{c}, *}$, P.Comba ${ }^{\mathrm{c}}$ and C.Daul ${ }^{\mathrm{a}}$}

${ }^{a}$ Departement de Chimie, Université de Fribourg, Chemin du Museé, 9, CH1700 Fribourg, Swizterland

${ }^{\mathrm{b}}$ Institute of General and Inorganic Chemistry, Bulgarian Academy of Sciences, Acad.G.Bontchev Str.B1.11, 1113 Sofia, Bulgaria ${ }^{c}$ Anorganisch-Chemisches Institut, Ruprecht-Karls-Universität Heidelberg, Im Neuenheimer Feld 270, D-69120 Heidelberg, Germany

In this presentation, we give a brief outline of a recently proposed approach for modeling ground and excited state electronic properties of transition metal complexes. It is based on a combination of Ligand Field theory, which is a classical tool for interpretation and modern density functional theory and it is shown that the new approach is equally suitable both for single nuclear $[1,2]$ and poly-nuclear transition metal complexes [3-4]. While ab-initio methods, such as multi reference CI have been applied with success to model ground and excited state properties of single nuclear complexes, magnetic exchange coupling still presents a challenge for ab-initio based methods. A parameterization of the ligand field for oligonuclear transition metal complexes allows to circumvent the size problem for calculating the exchange coupling constants and the magnetic properties, thus having a big impact for applications in molecular magnetism. In this talk a series of applications and test examples of magnetic exchange integrals will be given using experimentally well documented magnetic dinuclear complexes; these include bis$\mu$-hydroxo-bridged dimers of $\mathrm{Cu}(\mathrm{II})$ and oligonuclear cyanometallates. The impact of this approach will be discussed in close connection with ongoing experimental studies on these systems [5].

[1] M.Atanasov, C.A.Daul and C.Rauzy, Chem.Phys.Lett. 367, 737(2003).

[2] M.Atanasov, C.A.Daul, C.Rauzy, Structure and Bonding, 106,97(2004).

[3] M.Atanasov, C.A.Daul, Chem.Phys.Lett., 379, 209 (2003).

[4] M.Atanasov, C.A.Daul, Chem.Phys.Lett., 381, 584 (2003).

[5]M.Atanasov, P.Comba, Y.D.Lampeka, G.Linti, T.Malcherek, R.Miletich, A.I.Prikhod'ko and H.Pritzkow, Chem.Eur.J., submitted for publication.

Theoretical evaluation of NMR parameters for differently hydrogen bonded water molecules in the liquid state

$\underline{\text { Teemu S. Pennanen }}{ }^{1}$, Juha Vaara ${ }^{2}$, Perttu Lantto ${ }^{1}$ and Jukka Jokisaari ${ }^{1}$

Department of Physical Sciences ${ }^{1}$

University of Oulu, B.O. Box 3000, FIN-90014, Finland

Laboratory of Physical Chemistry, Department of Chemistry ${ }^{2}$

P.O.Box 55 (A. I. Virtasen aukio 1) FIN-00014, University of Helsinki, Finland

Various NMR parameters (chemical shift, shielding anisotropy, shielding asymmetry parameter, nuclear quadrupole coupling constant and asymmetry parameter) for oxygen and hydrogen/deuterium nuclei of differently hydrogen bonded molecules of liquid water are determined theoretically using snapshots from a CPMD ${ }^{1}$ simulation. Coordinate data of nuclei is fed into a quantum chemical program for the calculation of NMR parameters, using DFT/B3LYP-functional and for comparative purposes the HartreeFock level of theory. In the subsequent analysis, molecules are divided into groups according to the number of hydrogen bonds they possess, and the NMR property averages are calculated separately for each group. The division into groups also reveals the hydrogen bond distribution in liquid water, using a simple distance-based definition of hydrogen bonding. Distributions of the individual property values inside the groups show a wide range of possible values for individual nuclei, even for each hydrogen bonded species.

[1] R. Car, M. Parrinello, Phys. Rev. Lett. 1985, 55, 2471. 
Methodology and Applications

Stereoselective Synthesis of Chiral Bicyclic Lactams: A DFT Approach

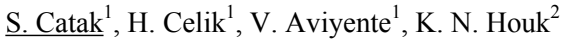

Chemistry Department ${ }^{1}$ Bogazici University, Bebek, Istanbul 34342 Turkey Department of Chemistry and Biochemistry ${ }^{2}$

University of California, Los Angeles, California 90095-1569

Chiral bicyclic lactams have been used as starting materials in the total synthesis of natural products ${ }^{1}$. These systems have provided access to a number of enantiomerically pure materials bearing quaternary carbon centers ${ }^{2}$. Homochiral 2-methylpyrrole derivatives (3) are synthesized in high yields starting from chiral amino alcohols (2) and 5-chloro-3-pentene-2-one (1). The photooxygenation of these compounds in the presence of a photosynthesizer furnishes the pyrrolooxazolone (5a and $\mathbf{5 b}$ ) structures in high diastereoselectivities ${ }^{3}$. In this study, B3LYP/6-31+G* has been used to understand the mechanism of the reaction as well as the factors responsible for the stereoselectivity. The ultimate goal of this research is to display the intermediates and transition states along this synthesis in order to guide experimentalists in their endavour.

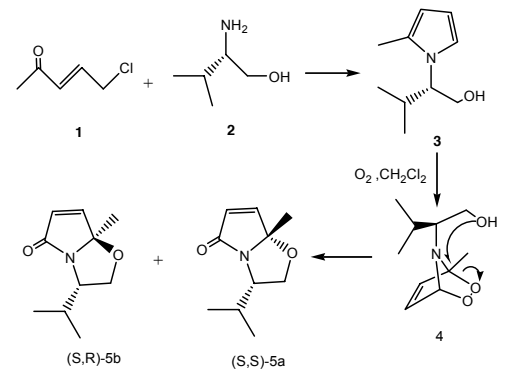

[1] D. Romo, J. L. Romine, W. Midura, Tetrahedron, 1990, 46, 4951.

[2] G. P. Brengel, A. I. Meyers, J. Org. Chem., 1996, 61, 3230.

[3] F. Aydogan, A.S. Demir, Tetrahedron: Asymmetry, 2004, 15, 259.

Methodology and Applications

\section{Regioselectivity in the $[2+2]$ photocycloaddition of triplet enones to substituted ethylenes : a Spin-Polarised Conceptual DFT Study}

\author{
F. de Proft, ${ }^{1}$ S. Fias, ${ }^{1}$ C. Van Alsenoy ${ }^{2}$ and P. Geerlings ${ }^{1}$
}

${ }^{1}$ Eenheid Algemene Chemie (ALGC), Vrije Universiteit Brussel (VUB), Faculteit Wetenschappen, Pleinlaan 2, 1050 Brussels, Belgium.

${ }^{2}$ Department of Chemistry, University of Antwerp, Universiteitsplein 1, B2610 Antwerp, Belgium

e-mail : fdeprof@vub.ac.be

In this contribution, a study of the regioselectivity of the photochemical $[2+2]$ cycloaddition of triplet enones with a series of ground state electronrich and electron-poor alkenes using DFT based reactivity descriptors is presented. Using the concepts of local softness combined with a local Hard and Soft Acids and Bases principle and a softness matching approach, the regioselectivity of this reaction can only be explained in the case of the interaction of the triplet enones with electron-rich alkenes.

In a second part, we have assessed the regioselectivity of this reaction within the framework of conceptual spin-polarised conceptual density functional theory, considering response functions involving the system's external potential $v$, number of electrons $N$ and spin number $N_{s}, N_{s}$ being the difference between the number of $\alpha$ and $\beta$ electrons in the spinpolarized system. Within this theory, we introduce the concepts of local spin philicity and donicity. Using the former, the regioselectivity can almost be completely interpreted as resulting from the interaction of the site on the alkene with the lowest spin-philicity (i.e. lowest destabilisation upon increasing spin number) with the site showing the highest change of spin number on the enone, expected to result in the largest stabilization of this species.
Methodology and Applications

P86

Interactions in Complex Materials: Ionic Molecular Crystals

Nicole A. Benedek ${ }^{\dagger \star}$ Ian K. Snook, ${ }^{\dagger}$ Kay Latham, ${ }^{\star}$ Irene Yarovsky ${ }^{\dagger}$

Departments of Applied Physics ${ }^{\dagger}$ and Applied Chemistry, ${ }^{\star}$

RMIT University, GPO Box 2476V, Melbourne 3001, Australia

The diverse range of elements and bonding scenarios present in molecular crystals means they often provide interesting 'laboratories' for the study of intermolecular interactions. Crystal structures are usually described in geometric terms, however it is the energetics of intermolecular interactions that determine the chemical and physical properties of molecular materials. Despite this, there is surprisingly little information on the energetics associated with the complex geometries which often occur in molecular crystals, particularly those containing metals and molecular ions.

An interesting family of novel molecular crystals, mixed-ligand copper organophosphonates, was recently synthesised in our laboratories [1]. We have been using Density Functional Theory in an effort to probe the electronic structure of these complex systems $[2,3]$ and thereby understand both the inter- and intramolecular interactions, in energetic and geometric terms, which determine the structure and properties. We also present preliminary findings concerning our use of the Quantum Monte Carlo (QMC) technique to study the fundamental physics of intermolecular interactions. QMC was the method used to parameterise the LDA and continues to be employed as an aid in designing more accurate functionals.

[1] Clarke, R., Latham, K., Rix, C., Hobday, M. and White, J., CrystEngComm, 2005, 7, 28.

[2] Benedek, N.A., Snook, I.K., Latham, K. and Yarovsky, I., J. Chem. Phys., 2005, Accepted.

[3] Benedek, N.A., Spencer, M.J.S., Latham, K. and Yarovsky, I., Chem. Phys. Lett., 2003, 378, 400.

Methodology and Applications

Molecular quantum similarity of enantiomers of amino acids.

$\underline{\text { G. Boon }}^{\S}$, C. Van Alsenoy* , F. De Proft ${ }^{\S}$, P. Bultinck\# and P. Geerlings ${ }^{\S}$

${ }^{\S}$ Free University of Brussels (VUB), Faculteit Wetenschappen, Eenheid Algemene Chemie (ALGC), Pleinlaan 2, B-1050 Brussels, Belgium.

*University of Antwerp (UA), Department of Chemistry, Universiteitsplein 1, B-2610 Antwerp, Belgium.

${ }^{\#}$ Ghent University (UGent), Department of Inorganic and Physical Chemistry, Krijgslaan 281 (S-3), B-9000 Ghent, Belgium.

In the framework of Computational and Conceptual Density Functional Theory $[1$, 2], molecular quantum similarity is evaluated for enantiomers in the case of molecules showing conformational flexibility, proposing the use of a Boltzmann weighted similarity index [3]. The conformers of the enantiomers of a series of amino acids were examined. Next to studying global similarity, using the already existing similarity indices defined by Carbó [4] and Hodgkin and Richards [5], we evaluated local similarity using our earlier proposed local similarity index [6] based on the Hirshfeld partitioning [7], in order to quantify the consequences of Mezey's Holographic Electron Density Theorem [8] in chiral systems.

Furthermore, the relation between the optical activity - an experimentally observable quantity - of L and D amino acids and their dissimilarity was studied.

\section{References}

[1] Parr, R. G. ; Yang, W. Density-Functional Theory of Atoms and Molecules, Oxford University Press, New York, Clarendon Press, Oxford, 1989.

[2] Geerlings, P. ; De Proft, F. ; Langenaeker, W. Chem. Rev. 2003, 103 (5), 1793.

[3] Boon, G. ; Van Alsenoy, C. ; De Proft, F. ; Bultinck, P. and Geerlings, P. , J. of Mol. Struct.: (THEOCHEM), accepted (January 2005).

[4] Carbó, R. ; Leyda, L. ; Arna, M. Int. J. Quantum Chem. 1980, 17, 1185.

[5] Hodgkin, E. E. ; Richards, W. G. Int. J. Quantum Chem. : Quantum Biology Symp. 1987, 14, 105.

[6] Boon, G. ; Van Alsenoy, C. ; De Proft, F. ; Bultinck, P. ; Geerlings, P. , J. Phys. Chem. A, 2003, 107, 11120 .

[7] Hirshfeld, F. L. Theor. Chim. Acta, 1977, 44, 129.

[8] Mezey, P. G. Mol. Phys. 1999, 96, 169. 
Methodology and Applications

pI258 ArsC: Reactivity and Stability of Anionic Compounds, Structural Aspects and Catalytic Activation of Electrophile and Nucleophile.

G. Roos ${ }^{\dagger}$, J. Messens ${ }^{\S}$, S. Loverix ${ }^{\S, \dagger}$, L. Wyns ${ }^{\S}$, F. De Proft ${ }^{\dagger}$ and $\underline{\text { P. Geerlings }} \underline{y}^{\dagger}$.

${ }^{\dagger}$ Vrije Universiteit Brussel (VUB), Eenheid Algemene Chemie (ALGC), Pleinlaan 2, B-1050, Brussels ${ }^{\S}$ Vrije Universiteit Brussel (VUB), Dienst Ultrastructuur, Pleinlaan 2, B-1050, Brussels.

Arsenate reductase (ArsC) catalyses the reduction of arsenate to arsenite by performing a nucleophillic attack of a cysteine (Cys10) on arsenate. The protonation state of the substrate is experimentally not attainable though indispensable for understanding of enzymatic catalysis. By performing a reactivity analysis within a Conceptual DFT context, using the HSAB principle, at a local level (softness matching procedure), an argumentation is built up to promote the nucleophilic attack of a thiolate on a dianionic rather than on a monoanionic substrate ${ }^{2}$. The reactivity analysis was performed in the gas phase where one is confronted with the instability of multiply charged anionic compounds with respect to electron emission. However, the departing electron has to overcome the repulsive coulomb barrier to escape. RCB values are calculated by a DFT based model ${ }^{3}$, which has the advantage to express the RCB in terms of molecular reactivity descriptors, describing the evolution of a system when passing from $\mathrm{N}$ - to $(\mathrm{N}-1)$-electrons and vice vers $a^{4}$. Starting from the X-ray structure of ArsC complexed with arsenite (PDB 1LJU), the 2-layer QM/QM ONIOM method provides a reliable structure of the Michaelis complex of ArsC. From this structure we have seen that of the numerous enzyme-substrate interactions, the hydrogen bond interaction between the substrate's leaving group and $\mathrm{N}_{\varepsilon} \mathrm{Arg} 16$ in particular has a positive influence on the electrophilicity of arsenate. Stabilization of Cys 10 is accomplished by the stabilization of its thiolate form by decreasing its $\mathrm{p} K_{\mathrm{a}}$ to 6.0 by both the macro-dipole of a nearby $\alpha$-helix and by the interaction network from $\mathrm{S} \gamma \mathrm{Cys} 10$ via $\mathrm{HO} \gamma \mathrm{Ser} 17$ and $\mathrm{HN}_{\delta} \mathrm{Asn} 13$ to potassium ${ }^{5}$.

[1] Zegers I., Martins J.C., Willem R.,Wyns L., Messens J., Nature Struct. Biol. 2001, 8, 843. [2] Roos, G., Loverix, S., De Proft, F., Wyns, L. Geerlings P., J. Phys. Chem. A 2003, 107, 6828. [3] Langenaeker, W., De Proft, F., Tielens, F., Geerlings, P., Chem. Phys. Lett. 1998, 228, 628. [4] Roos, G., De Proft, F., Geerlings P., J. Phys. Chem. A 2005, 109, 652. [5] Roos, G., Messens, J., Loverix, S., Wyns, L., Geerlings P., J. Phys. Chem. $B$ 2004, 108,17216

Methodology and Applications

Two Types of Complexes of Carbon Monoxide with the Hydroxyl Group and the Reproduction of the CO Frequency Shifts by MP2 and B3LYP methods

$$
\text { Igor Ignatyev }^{1} \text { and Tom Sundius }{ }^{2}
$$

${ }^{1}$ Department of Chemistry, Radiochemistry Laboratory, St. Petersburg State University, St. Petersburg 199034, Russia

${ }^{2}$ Department of Physical Sciences, University of Helsinki, P.O. Box 64, FIN-00014 Helsinki, Finland

Carbon monoxide may form two types of hydrogen bonding with the $\mathrm{OH}$ group, i.e. OH. .CO (I) and OH. ..OC (II). They are characterized by different signs of $\mathrm{CO}$ frequency shifts in complexes with water [1] and with surface silanol groups [2]. MP2 satisfactorily describes the blue shifts for complex I, but fails to reproduce experimental values of the red shifts for complex II. B3LYP describes them substantially better and shows that in the series $\mathrm{ROH}\left(\mathrm{R}=\mathrm{H}, \mathrm{CH}_{3}, \mathrm{SiH}_{3}\right)$ both shifts increase on going from water to silanols in accord with experimental results. However, B3LYP predictions for $\mathrm{CO}$ complexes with silanols $\mathrm{R}_{3} \mathrm{SiOH}(\mathrm{R}=\mathrm{H}, \mathrm{F}, \mathrm{OH})$ give the $2: 1$ ratio of shifts, in contrast to the $1: 1$ ratio observed for surface species. The high red shift observed for surface species with $\mathrm{SiOH}$. . OC bonding may be reproduced only in the cases when the interaction of $\mathrm{CO}$ with atoms imitating lattice oxygens is present.

[1] J. Lundell, M. Räsänen, J. Phys. Chem., 1995, 99, 14301.

[2] P.Yu. Storozhev, C. Otero Areán, E. Garrone, P. Ugliengo, V.A. Ermoshin, and A.A. Tsyganenko, Chem. Phys. Lett. 2003, 374, 439
Methodology and Applications

\section{The Study of Corrosion Inhibition of Amides and Thiosemicarbazones by MNDO and DFT Calculations}

\author{
$\underline{\text { Fatma Kandemirli }} \underline{1}$ and Seda Sagdinc ${ }^{2}$
}

Kocaeli University, Faculty of Science, Dept. of Chemistry, Kocaeli ${ }^{1}$ Kocaeli University, Faculty of Science, Dept. of Physics, Kocaeli, Turkey. ${ }^{2}$

Amides and thiosemicarbazones are recognized corrosion inhibitors ${ }^{1}$. The efficiency of organic compounds as a corrosion inhibitor is closely related to molecular structure. DFT and semi-empirical calculations have been widely used to study the relation between corrosion inhibitor effect and molecular structure $^{2}$.

In the present investigation, molecular structures and electronic properties of urea (U), thiourea (TU), acetamide (A), thioacetamide (TA), semicarbazide (SC), thiosemicarbazide (TSC), methoxybenzaldehyde thiosemicarbazone (MBTSC), 2-acetylpyridine-(4-phenyl)thiosemicarbazone (2AP4PTSC), 2acetylpyridine-(4-methyl)thiosemicarbazone (2AP4MTSC), benzoin thiosemicarbazone (BZOTSC) and benzil thiosemicarbazone (BZITSC) were calculated with semi-empirical (MNDO) and DFT methods. It was found that the molecules which include a thiocarbonyl group, e.g. TU, TA and TSC have higher inhibition efficiency than the corresponding compounds $^{3}$. It has been found correlation between corrosion inhibition efficiencies and electronic properties of these molecules. All the calculations were carried out with the help of complete geometry optimization. MNDO and DFT calculations were performed GAUSS 98W program package (Version 5.4 RevA -9).

[1] M.Özcan, İ. Dehri, Prog. Org. Coat., 2004, 51, 181

[2] J. Fang, J. Li, J. Mol. Struct., 2001, 571, 139.

[3] E.E. Ebenso, et al., Mater. Chem. Phys., 1999, 60, 79

Methodology and Applications

P92

A time-dependent density functional theory study of one- and twophoton absorption in donor-acceptor chromophores

Paul N. Day, Kiet A. Nguyen, and Ruth Pachter

Air Force Research Laboratory, Materials \& Manufacturing Directorate, AFRL/MLPJ, Wright-Patterson Air Force Base, Ohio, USA 45433-7702

In our ongoing theoretical studies to predict the photophysical properties of materials of interest, we report the results for one-photon, and twophoton absorption (OPA, TPA) spectra, for a series of chromophores, in which electron donating and accepting groups are attached to a core having a $\pi$-delocalized electron structure, such as stilbene or fluorene. Linear response time-dependent density functional theory, with hybrid exchange-correlation functionals, was applied in all calculations. We find that the calculated excitation energies are generally in good agreement with experiment, particularly when compared to measurements carried out in a nonpolar solvent. Predicted TPA crosssections, applying the two-state approximation, are also in relatively good agreement with experiment; however, a lack of experimental systematic solvent effects data limits a detailed comparison as yet. 
Methodology and Applications

Electronic structure calculation of the perovskite $\mathrm{La}_{1-x} \mathrm{Ca}_{x} \mathrm{CoO}_{3}$ as a function of Ca substitution

$$
\text { M. Sahnoun }{ }^{1, \star}, \text { C. Daul }{ }^{1} \text {, O. Haas }{ }^{2}
$$

${ }^{1}$ Department of Chemistry, University of Fribourg, CH-1700 Switzerland

${ }^{2}$ Paul Scherrer Institut, CH-5232 Villigen PSI, Switzerland

$\mathrm{La}_{1-x} \mathrm{~A}_{x} \mathrm{CoO}_{3}$ compounds, $\mathrm{A}=\mathrm{Ca}, \mathrm{Sr}, \mathrm{Ba}$, are particularly intriguing since they undergo several changes in their electronic and magnetic properties as function of temperature and/or divalent substitution. The rich magnetic and electronic phase diagram of $\mathrm{LaCoO}_{3}$ is a function of the crystal field splitting, $\Delta_{c f}$ and of the exchange energy, $\Delta_{e x}$, for $\mathrm{Co}^{3+}$ $\left(3 \mathrm{~d}^{6}\right)$ ions in an octahedral environment. Thus, either the low-spin (LS) $\left(\mathrm{t}_{2 g}^{6} \mathrm{e}_{g}^{0}, \mathrm{~S}=0\right)$ or the intermediate-spin (IS) $\left(\mathrm{t}_{2 g}^{5} \mathrm{e}_{g}^{1}, \mathrm{~S}=1\right)$ configuration appears to be energetically more favorable than the Hund's rule predicted high-spin (HS) state $\left(\mathrm{t}_{2 g}^{4} \mathrm{e}_{g}^{2}, \mathrm{~S}=2\right)$. In the hole-doped compounds, $\mathrm{La}_{1-x} \mathrm{~A}_{x} \mathrm{CoO}_{3}$, the complexity of the system is further increased by the occurrence of $\mathrm{Co}^{4+}\left(3 \mathrm{~d}^{5}\right)$ ions which are susceptible to exist in several spin-configurations as well. The electronic structure of the perovskite $\mathrm{La}_{1-x} \mathrm{Ca}_{x} \mathrm{CoO}_{3}$ is obtained as a function of $\mathrm{Ca}$ substitution from generalized gradient-corrected, full-potential, spin-density-functional band structure calculations. The doping changes the valence states of Co to give a mixture of $\mathrm{Co}^{3+}$ and $\mathrm{Co}^{4+}$.

In the present work we report the energetics of various spin-states of Co as a function of hole doping in $\mathrm{LaCoO}_{3}$. The energetic of different spin configurations are estimated using the fixed-spin-moment (FSM) method. Based on these results we will consider the possible spin-transition in $\mathrm{LaCoO}_{3}$ upon hole doping.

Methodology and Applications

Density Functional Investigation of the Observed Low

Exchange Magnetic Coupling of a New Synthesized Rare Asymmetric End-On Double Azido-Bridged Cu(II) Complexes

Mohamed Zbiri ${ }^{a}$, Subratanath Koner $^{b}$, Claude Daul ${ }^{a}$

${ }^{a}$ Department of chemistry, University of Fribourg -Pérolles,CH-1700 Fribourg, Switzerland

${ }^{b}$ Department of Chemistry, Jadavpur University, Jadavpur, Calcuta 700 032, India

Quantum chemical calculations based on density functional theory (DFT) are performed on some new synthesized complexes in order to investigate their unexpected observed weak exchange magnetic coupling. Within broken symmetry (BS) density functional formalism, calculations on these systems are carried out, and the so-called hybrid method using B3LYP hybrid functional is used and it produces a very good estimation [1] of the exchange coupling constants as compared to the experimental data.

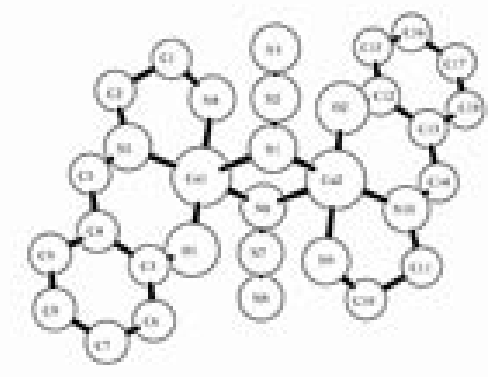

[1] M. Zbiri et al., In preparation
Methodology and Applications

P94

Torsional potential of $\pi$-conjugated molecules using the Localized Hartree-Fock Kohn-Sham exchange potential

Eduardo Fabiano and Fabio Della Sala

National Nanotechnology Laboratory of INFM

Università degli Studi di Lecce, Via per Arnesano, 73100 Lecce, Italy

Despite the many successes achieved by Density-Functional Theory (DFT) in many areas of chemistry, the quantitative evaluation of the torsional potential of $\pi$ conjugated systems still poses particularly difficult problems, due to the small energy differences that need to be computed. Usual exchange-correlation (XC) functional show several shortcomings [1]. In this work we use the Localized Hartree-Fock (LHF) [2] method to study the torsional potential of $\pi$-conjugated molecules. The LHF method is an effective exact-exchange approach, it is parameter free, numerically stable and it has the same computational cost of an hybrid DFT. We compare LHF results with results from Hartree-Fock and correlated post Hartree-Fock methods as well as from other commonly used DFT approximations, for three model molecules: butadiene, bithiophene and biphenyl.

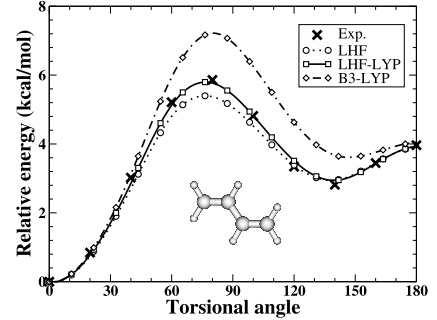

We found that LHF can give an accurate description of the torsional potential of $\pi$-conjugated molecules. In addition the description of the torsional potential with the LHF method can be improved with the addition of a correlation term (see Figure), in contrast to other exchange functionals.

[1] J. C. Sancho-Garcia et al., J. Chem. Phys., 2004, Vol, 121, 3096

[2] F. Della Sala and A. Görling, J. Chem. Phys, 2001, Vol, 115, 5718.

Methodology and Applications

P96

Application of the density functional theory derived orbital-free embedding potential to calculate the splitting energies of lanthanide cations in chloroelpasolite crystals

Mohamed Zbiri ${ }^{a}$, Tomasz A. Wesolowski ${ }^{b}$, Claude Daul $^{a}$

${ }^{a}$ Department of chemistry, University of Fribourg -Pérolles,CH-1700 Fribourg, Switzerland

${ }^{b}$ Department of chemistry, University of Geneva -30, quai Ernest-Ansermet, CH-1211 Genève 4, Switzerland

Ligand field splitting energies of lanthanides $\mathrm{Ln}^{3+}(\mathrm{Ln}=$ from Ce to $\mathrm{Yb})$ in octahedral environment are calculated using the first-principles based embedding formalism [1]. In this formalism, the lanthanide is described at orbital level whereas its environment is represented by means of an additional term (effective embedding potential) in the Kohn-Sham-like one-electron equations. This term is expressed as an explicit functional of two electron densities: that of the cation $\rho_{1}$ and that of the ligands $\rho_{2}$. It is shown that the formalism and the applied relevant approximate functionals, developed and used previously for embedded subsystems comprising only for s-, p-, and d-elements, is also an adequate method [2] for embedded f-elements. The calculated splitting energies in the studied systems are attributed to two main factors $i$ ) polarization of the electron density of the ligands and ii) ion-ligand Pauli repulsion.

[1] T. A. Wesolowski and A. Warshel, J. Phys. Chem., 1993, 97, 8050

[2] M. Zbiri et al., Chem. Phys. Lett, 2004, 397, 441. 
Methodology and Applications

Modelling the Cyclocondensations of $\alpha$-ketophosphonoenoates with Dienes

N. Celebi-Olcum, V. Aviyente

Chemistry Department Bogazici University, Bebek 34342 Istanbul, Turkey

The reaction of diethyl $\alpha$-crotonylphosphonate (1) with cyclopentadiene, in the absence of a Lewis catalyst, leads to the formation of the hetero-DielsAlder product (2) in addition to the normally expected cycloadduct (3) with poor stereoselectivity. ${ }^{1}$ The addition of Lewis acid $\left(\mathrm{SnCl}_{4}\right)$ favors the heteroDiels-Alder product (2) and enhances endo/exo selectivity of the major product. ${ }^{1,2}$ Changing cyclopentadiene to cyclohexadiene in the presence of Lewis acids has a net effect on product distribution. The reaction does not show any selectivity between normal and hetero-Diels-Alder product. In this study, the reaction of dialkyl $\alpha$-keto- $\gamma, \beta$-unsaturated phosphonates with cyclopentadiene and cyclohexadiene will be modelled with B3LYP/6-31G* in the absence and in the presence of Lewis acids, in order to understand the origin of the stereoselectivity.

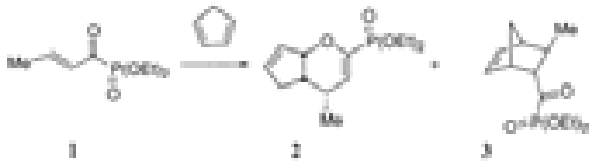

[1] S. Hanessian, P. Compain, Tetrahedron, 2002, 58, 6521.

[2] L. A. Telan, C. D. Poon, S.A. Evans Jr., J. Org. Chem., 1996, 61, 7455 .

Methodology and Applications

Density Functional Theory Study of Vibrational Spectrum of Sertraline

\section{$\underline{\text { Seda Sagdinc }}{ }^{1}$, Fatma Kandemirli², Sevgi Bayari ${ }^{3}$}

${ }^{1}$ Kocaeli University, Faculty of Science, Dept. of Physics, Kocaeli,Turkey ${ }^{2}$ Kocaeli University, Faculty of Science, Dept. of Chemistry,Kocaeli,Turkey ${ }^{3}$ HacettepeUniversity,Faculty of Education, Dept of Physics, Ankara, Turkey

Sertraline hydrochloride (4-(3,4-dichlorophenyl)-1,2,3,4,-tetrahydro- $N$ methyl-1-naphthalenamine hydrochloride is a highly potent and selective inhibitor of serotonin $(5 \mathrm{HT})^{1}$. It is a basic compound and of pharmaceutical application for antidepressant treatment. Density functional theory (DFT) calculations using Becke's exchange in conjunction with Lee-Yang-Parr's correlation functional (BLYP), Becke's three-parameter hybrid DFT/HF method using Lee-Yang-Parr's correlation functional (B3LYP) and ab initio Hartree-Fock (HF) method have been carried out to investigate the structure and vibrational spectrum of sertraline. The IR spectrum of sertraline is recorded in the solid state. On the basis of the comparison between calculated and experimental results and also the comparison with related molecules, assignments of fundamental vibrational modes are examined. Structural parameters obtained by B3LYP/6-31G* geometry optimization are in good agreement with available experimental data ${ }^{2}$ (see figure).

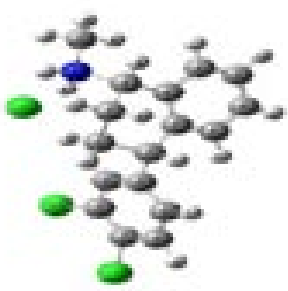

[1] A. Novoselsky, R. Glaser, Magn. Reson. Chem., 2002, 40, 723.

[2] R.G. Parr, W.Yang, Density-Functional Theory of Atoms and Molecules, 1989, Oxford Univ. Press, Oxford.
Methodology and Applications

DFT Calculations on TtBP- a Highly-distorted Porphyrin with an Unusual Resonance Raman Spectrum.

Roma E Oakes, Steven E J Bell.

The Queen's University of Belfast, School of Chemistry, David Keir Building, Stranmillis Road, Belfast. BT9 5AG.

Porphyrins are an incredibly diverse class of compounds. They have applications in areas such as solar-energy conversion and photodynamic therapy and, in nature, they lie at the heart of the reactions that drive photosynthesis. Most porphyrins have planar or near-planar macrocycle cores, but over the last 10 years there has been an explosion in the number of crystal structures of non-planar porphyrins. In general, the distorted compounds have much shorter excited state lifetimes than their planar analogues and some display strongly temperature-dependent photophysical behaviour. This raises the possibility that the excited state properties of porphyrins may be "tuned" by introducing distortions into the macrocycle but, of course, such an approach requires an understanding of how the nature of the distortions is linked with the photophysics.

This talk describes DFT calculations on non-planar porphyrins such as free-base tetra-tert-butyl porphyrin (T $t \mathrm{BP})$. The resonance Raman spectrum of $\mathrm{T} t \mathrm{BP}$ has unusually strong, low-frequency bands which do not appear in the spectra of very similar, but planar near-analogues. However, even with extensive experimental data it was not possible to explain why these bands appear. The calculations show that they originate in vibrations that involve folding of the pyrrole parts of the ring [1]. This was unexpected, because this type of out-of-plane vibration is not normally seen in porphyrins, but it implies that the distortions in the excited-state are along coordinates that are different to those in the ground state, where each of the $\mathrm{C}_{\mathrm{m}}$-pyrrole- $\mathrm{C}_{\mathrm{m}}$ units in T $t \mathrm{BP}$ is near-planar. In the excited-state it is the $\mathrm{C}_{\mathrm{m}}$ pyrrole- $C_{m}$ units themselves that are twisted. These deformations may be the origin of the unusual photophysical properties of $\mathrm{T} t \mathrm{BP}$, in particular its anomalously short excited-state lifetime.

[1] Oakes, R.E., S.J. Spence, and S.E.J. Bell, J Phys Chem. 2003, 107, 2964.

Methodology and Applications

P100

\section{Structural Optimization of Distorted Octahedral Cobalt(III) Complexes with a Carbonato Ligand}

\section{Toshiaki Taura and Tomokazu Kobayashi}

Faculty of Information Science and Technology

University of Aichi Prefecture, Nagakute, Aichi 480-1198, Japan

The $\mathrm{d}^{6}$ cobalt(III) complex does not display a striking structural deformation from octahedral geometry. One of the exceptions is the cobalt(III) carbonato complex with a $\mathrm{CO}_{3}{ }^{2-}$ chelate, which shows a great deviation of the $\mathrm{O}-\mathrm{Co}-\mathrm{O}$ bond angle of $70^{\circ}$ from the octahedral one $\left(90^{\circ}\right)$.

This is due to the steric restriction

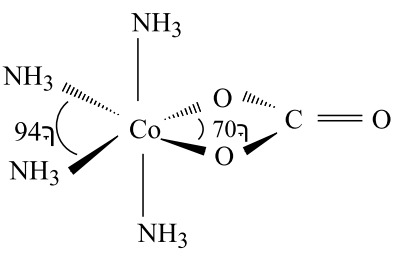
of the chelate carbonato ligand of a four membered ring. This deformation should supply some strain for the cobalt(III) complex and influence the structure around the cobalt ion and the reactivity concerning this complex. From this viewpoint, some experimental approaches have already been carried out ${ }^{1}$. One of the examples is the trans influence of a chelated carbonate. Any distinct influence was not detected in the $\mathrm{Co}-\mathrm{N}$ bond length trans to the $\mathrm{Co}-\mathrm{O}$ bond but the $\mathrm{N}-\mathrm{Co}-\mathrm{N}$ bond angle trans to the $\mathrm{O}-\mathrm{Co}-\mathrm{O}$ angle becomes larger than the octahedral angle $\left(90^{\circ}\right)^{2}$. In this work, we determined the structures, bond lengths and angles for several carbonato cobalt(III) complexes by using the DFT calculation at B3LYP/6-31G* level. After comparing the DFT and X-ray structures of these complexes, we discussed about the calculation reliability of this method for the cobalt(III) complexes having the strain in the structure.

[1] R. J. Dobbins, G. M. Harris, J. Am. Chem. Soc., 1970, 92, 5104

[2] T. Taura, Inorg. Chim. Acta, 1989, 158, 33 
Methodology and Applications

The structure and properties of small Co clusters

Vebjørn Bakken ${ }^{1,2}$, Ole Swang ${ }^{2}$, Unni Olsbye ${ }^{1}$ and Erling Rytter ${ }^{3}$

1) Department of Chemistry, University of Oslo, P.O. Box 1033 Blindern, $\mathrm{N}-0315$ Oslo, Norway

2) SINTEF, Materials and Chemistry, P.O. Box 124 Blindern,

$\mathrm{N}-0314$ Oslo, Norway

3) Statoil R\&D, N-7005 Trondheim, Norway

Cobalt is a transition metal with important catalytic properties. To better understand how Co-based catalysts actually work, a detailed model at the molecular level is needed. Using DFT methods, we have investigated the structure and properties of small cobalt clusters $\mathrm{Co}_{n}(n=1-6)$

The neutral Co atom has three unpaired electrons. As one starts building up clusters, these electrons pair up only to a very limited degree, yielding wave functions with very high multiplicity. Converging the wave functions and also the geometries of such species is a challenge, which may be why there is a striking scarcity of calculations on these systems in the literature.

We have investigated the performance of different functionals and a few different basis sets, to determine a suitable level of theory. Extensive calculations have been carried out to determine the lowest energy of different spin states and spatial symmetries for each cluster size considered.

Methodology and Applications

P103

\section{Dynamics of Argon in confined geometry}

K. Schmalzl ${ }^{1}$, M. Rheinstädter ${ }^{2}$ and D. Strauch ${ }^{3}$

${ }^{1}$ Forschungszentrum Jülich, 52425 Jülich, Germany ${ }^{2}$ Institut Laue-Langevin, BP 156 - 38042 Grenoble Cedex 9, France ${ }^{3}$ Institut für Theoretische Physik, Universität Regensburg, Germany

Condensed matter in geometrical confinement like nanoporous matrices allows to study the change of dynamics due to spatial restrictions. We investigated ${ }^{36}$ Argon adsorbed in nanoporous Gelsil Glass by inelastic neutron scattering. At low filling fractions the atoms form an amorphous adsorbate film on the pore walls. At higher fillings, a capillary condensate forms in the pore center. The preparation of single or of several monolayers were possible what permitted the study of, e.g., the dynamical interaction between the third and second layer.

We compare the measured density of states of different fillings with the ones calculated with ab initio calculations. The calculations were done with LDA and GGA pseudopotentials. Although we encountered several problems because of the van der Waals character of the Argon bindings we could reproduce several experimental results. One crucial point for the theoretical description is the expected energy gap because of quantisation of the phonon modes in the plane of the pore.
Methodology and Applications

\section{Approaching anharmonic properties in fluorites}

K. Schmalzl ${ }^{1}$ and D. Strauch ${ }^{2}$

${ }^{1}$ Forschungszentrum Jülich, 52425 Jülich, Germany

${ }^{2}$ Institut für Theoretische Physik, Universität Regensburg, Germany

The linear properties of crystals like elastic constants and vibrational frequencies are well understood qualitatively and, thanks to developments of density functional response methods, also quantitatively. For years the inelastic neutron scattering (INS) method has supplied the necessary detailed information for a comparison. While qualitatively understood likewise, the nonlinear effects like thermal expansion or conductivity and other temperature dependent properties are still awaiting quantitative explanation. The anharmonic inter-atomic potential shows up in various properties, and the most detailed information can be gained from the study of the dependence of the phonon excitations due to temperature, pressure, or other external fields.

The ionic conduction of $\mathrm{CaF}_{2}$ is based on the interionic potential with low energy barriers and thus with strong anharmonicity. In order to gain more insight into this anharmonicity we have carried out an INS study of the temperature dependence of all modes with wavevectors along the main symmetry directions. We observed unusually large line shifts and broadening reaching for many modes the overdamped case at higher temperatures.

To leading order, the shift is composed of three different anharmonic processes involving third- and fourth-order anharmonicity, and for the sake of comparison with theoretical results it is necessary to disentangle these three processes. At present, the DFT calculation of all of the three processes is out of reach. One of these processes is the effect of volume change, either due to thermal expansion or due to pressure. Using densityfunctional methods we have calculated the effect of volume change on the mode frequencies and their derivatives and obtained large modeGrüneisen parameters. The calculated energy dependence of the channels for anharmonic decay are compared with the measured phonon widths. For comparison the effect of thermal expansion has been investigated for another fluorite ionic conductor, $\mathrm{BaF}_{2}$.

Methodology and Applications

Substituent effects on the mechanism of indigo dyes formation: Time Dependent Density Functional Theory Study.

Al Mokhtar Lamsabhi ${ }^{*}$, Carlos A. Escobar and Patricia Pérez

Departamento de Química, Facultad de Ecología y Recursos Naturales, Universidad Andrés Bello, Av. Republica 275, Santiago, Chile.

Indigo is one of the oldest known natural dyes which present a great interest in the coloration industry. It is biosynthesised from the reaction between the protected indoxil and its oxidation product, indoleinone, as described in the following model scheme:

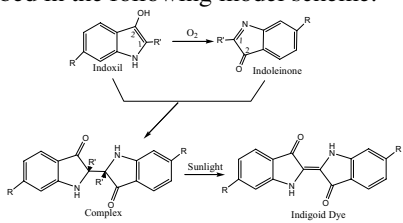

Density functional Theory at $6-31+\mathrm{G}^{* *}$ level has been used to study the formation of indigo and its derivatives starting from the indoleinone and indoxil precursors. In the present study, the substituent effect on the electrophilic and nucleophilic character of carbon atom at position 1 , have been evaluated using the local electronic fukui function responses. In order to estimate the change on the electronic spectra issued from the complexation, TD-DFT have been carried out at the same level of theory.

A good correlation has been found between experimental and theoretical absorption wavelengths of studied dyes. The most important effect on the interaction between indoleinone and indoxil to form indigo dyes is attached to substituent field effect when the Taft's model is used.

Acknowledgements: We thanks to Universidad Andrés Bello, grants DI-41-04, DI-15-04, and DI-17-04. We are grateful to the Millennium Nucleus for Applied Quantum Mechanics and Computational Chemistry, grant $\mathrm{N}^{\circ} \mathrm{P} 02-004-\mathrm{F}$ (Mideplan and Conicyt). This work has been also supported by Fondecyt, grant No. 1020069 .

Permanent Adress: Departamento Quimica C-9, Facultad de Ciencias, Universidad Autónoma de Madrid, Cantoblanco, E-28049 Madrid, Spain. 
Methodology and Applications

Density Functional Theory as a Means for the Discovery of Mechanistic Pathways for the Catalytic Synthesis of Methanol on $\mathrm{Cu}$.

\section{Beatrice Nikolaidi, Steven Y Liem, Julian HR Clarke}

School of Chemistry, University of Manchester, Oxford Road, Manchester, M13 9PL, United Kingdom

The annual consumption of methanol exceeds 25 million tons. Syn-gas is used as a feedstock for the production of methanol over $\mathrm{Cu}$-based catalysts. Although the main reaction pathways in this multi-stage process have been described experimentally, many mechanistic details remain obscure.

Using DFT, we have been able to contribute to the characterisation of several interesting mechanisms such as the adsorption of $\mathrm{H}$ on $\mathrm{Cu}$, the adsorbate induced reconstruction of the catalyst, the source of the energy barrier on the formation of $\mathrm{HCO}_{2}$ and the intermediates in the conversion $\mathrm{HCO}_{2}$ to $\mathrm{H}_{2} \mathrm{CO}$. The adsorption energies and conformations of the reaction intermediates as well as the active sites of the catalyst were calculated by means of a plane wave basis set and the exchange correlation effects described by means of the Perdew-Wang (PW91)[1] functional within the context of the generalized gradient approximation (GGA). Nudged Elastic Band method (NEB)[2] was used to locate the transition states and determine the activation energies and minimum energy pathways (MEP) for the reactions. In particular, we have shown that on perfect and also on $2 \mathrm{x} 1$ reconstructed $\mathrm{Cu}(110)$ surfaces the controlling step in $\mathrm{H}+\mathrm{CO}_{2}$ reaction is the formation of a C-H bond followed by a near $120^{\circ}$ rotation of the intermediate. The formate product is strongly bound to $\mathrm{Cu}$ through the $\mathrm{O}$ atoms. Where comparisons can be made, adsorption energies and energy barriers are in very good agreement with experiment. The $\mathrm{Cu}$ surface was modelled using the slab supercell approach with 3D periodic boundaries and the Brillouin zone (BZ) sampling based on the Monkhorst-Pack technique[3].

[1]. J. P. Perdew, et al. Phys. Rev. B 1992, 46, 6671

[2]. G. Mills, et al. Surface Science 1995, 324, 305

[3]. H. J. Monkhorst, et al. Phys. Rev. B 1972, 13, 5188.

Methodology and Applications

Magnetic and electric properties of bismuth-containing perovskites

$\underline{\text { Pio Baettig }}^{1,2}$, Nicola Spaldin ${ }^{1}$, Claude Daul ${ }^{2}$

${ }^{1}$ Materials Department, University of California, Santa Barbara CA, 93106, USA.

${ }^{2}$ Département de chimie, Université de Fribourg, Pérolles, 1700 Fribourg, $\mathrm{CH}$

Multifunctional materials that combine a spontaneous magnetization with a ferroelectric polarization are of tremendous fundamental interest. [1,2]

Such materials show a variety of interesting properties, especially combined ferromagnetism and ferroelectricity in $\mathrm{BiMnO}_{3}$. We report first principles calculations on a variety of Bi-containing perovskite compounds, including $\mathrm{Bi}_{2} \mathrm{FeCrO}_{6},[3] \mathrm{BiAlO}_{3}$, and $\mathrm{BiGaO}_{3},[4]$ and compare their electrical and magnetic properties.

We report the influence of the application of a Hubbard- $U$ on the structural and magnetic properties and depict this "functio-structural dependence" for the example of $\mathrm{Bi}_{2} \mathrm{FeCrO}_{6}$. (cf. Figure 1) There, a local density approximation calculation suggests a low-spin ar-

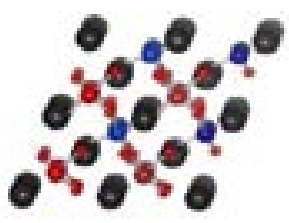

Figure 1: Structure of $\mathrm{Bi}_{2} \mathrm{FeCrO}_{6}$. The Bi-ions are represented in black. the Fe-ions in red and the Cr-ions in blue.

rangement of the spins on the Fe. Application of a Hubbard- $U$ leads to a low-spin to high-spin transition and an augmentation of the unit-cell size.

[1] M. Fiebig et. al. Nature, 2002, 419, 818-820.

[2] H. Schmid, Ferroelectrics, 1994, 162, 317-338.

[3] P. Baettig, N. A. Spaldin, Appl. Phys. Lett., 2005, 86, 012505

[4] P. Baettig, C. F. Schelle, R. LeSar, U. V. Waghmare, N. A Spaldin, Chem. Mater., in press, 2005, cm0480418
Methodology and Applications

P106

Organic SIMS: coupling between vibrational and electronic modes of sputtered molecules.

V. Solomko', M. Verstraete', A. Delcorte', B.J. Garrison', X. Gonze' and P.Bertrand

Unité de Physico-Chimie et de Physique des Matériaux', Université

Catholique de Louvain, Croix du Sud 1, Louvain-la-Neuve B-1348, Belgium

Department of Chemistry 2 , Penn State University, 512 Davey Laboratory, University Park, PA 16802, USA

This study aims at improving the quantitative analysis of organic samples in SIMS. In the previous article [1] we investigated various channels of internal energy dissipation for excited sputtered molecules. These investigations should help us to understand the specifics of the conversion of vibrational energy into electronic excitation.

The organic molecule [1,2,3,4-tetraphenylnaphthalene (TPN)], consisting of several phenyl rings and potentially reflecting the common processes in organic SIMS, has been analyzed using the first-principle methods in order to check whether TPN molecules can relax by electron emission.

We conclude that ionization due to the transformation of the internal vibrational energy of molecules appears to be a universal way of energy dissipation of a sputtered organic particle, weakly dependent on the molecule being investigated.

[1] V.Solomko, A.Delcorte, B.J.Garrison and P.Bertrand, Applied Surface Science. 2004, 231-232, 48-53.

Methodology and Applications

P108

Modeling the Copper(II)bisoxazoline Catalyzed Claisen Rearrangement of 2-Substituted Allyl Vinyl Ethers.

\section{Cem-Ozturk $^{1}{ }^{1}$ Viktorya Aviyente ${ }^{1}$, Ian Hillier $^{2}$, Mark A Vincent ${ }^{2}$}

${ }^{1}$ Chemistry Department Bogazici University, Bebek 34342 Istanbul, Turkey ${ }^{2}$ Chemistry Department University of Manchester, M13 9PL Manchester, United Kingdom

Copper(II)bisoxazolines are found to be useful catalysts for the Claisen rearrangement of 2-substituted allyl vinyl ethers. The highly diastereoselective generation of two vicinal chiral centers is an inherent strength of the thermal aliphatic Claisen rearrangement. The diastereoselectivity of the thermal Claisen rearrangement was combined with the ability of $[\mathrm{CuII}(\mathrm{box})]$ complexes to catalyze the Claisen rearrangement enantioselectively. $[\mathrm{Cu}\{(\mathrm{S}, \mathrm{S})-\mathrm{Ph}-\mathrm{box}\}](\mathrm{OTf})_{2}(\mathbf{1})$ was used as a catalyst for the Claisen rearrangement of 2-alkoxycarbonyl-substituted allyl vinyl ethers containing two stereogenic double bonds (Scheme 1). Reversal of stereoselectivity is proposed with $[\mathrm{Cu}\{(S, S)-t \mathrm{Bu}-\mathrm{box}\}](\mathrm{OTf})_{2}$ catalyst. The purpose of this study is to find the reason for the streoselectivity and investigate further effects of $[\mathrm{Cu}\{(\mathrm{S}, \mathrm{S})-\mathrm{Ph}-\mathrm{box}\}](\mathrm{OTf})_{2}$ and $[\mathrm{Cu}\{(S, S)-t \mathrm{Bu}-$ box $\}](\mathrm{OTf})_{2}$ catalysts on these Claisen rearrangements via the UB3LYP/6$31 \mathrm{G}^{*}$ method.

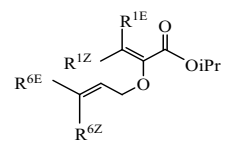

$\mathrm{R}^{1 \mathrm{E}}=$ methyl, $\mathrm{R}^{6 \mathrm{Z}}=\mathrm{n}$-propyl $\mathrm{R}^{1 \mathrm{Z}}=$ benzyl, $\mathrm{R}^{6 \mathrm{Z}}=\mathrm{n}$-propyl Scheme 1. Allyl vinyl ethers used for the Claisen rearrangement catalyzed by Lewis acid
$[\mathrm{Cu}\{(\mathrm{S}, \mathrm{S})$-Ph-box $\}](\mathrm{OTf})_{2}$ and $[\mathrm{Cu}\{(\mathrm{S}, \mathrm{S})$-tBut-box $\}](\mathrm{OTf})_{2}$ $\mathrm{R}^{1 Z}=$ methyl, $\mathrm{R}^{6 \mathrm{Z}}=\mathrm{n}$-propy

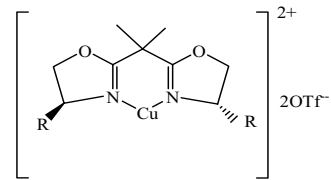

R=Ph,(S,S)- : $\left[\mathrm{Cu}^{\mathrm{II}}\{(\mathrm{S}, \mathrm{S})-\mathrm{Ph}-\mathrm{box}\}\right](\mathrm{OTf})_{2}$ $\mathrm{R}=\mathrm{tBut}, \mathrm{S}, \mathrm{S})-:\left[\mathrm{Cu}^{\mathrm{uI}}\{(\mathrm{S}, \mathrm{S})-\mathrm{But}-\mathrm{box}\}\right](\mathrm{OTf})_{2}$ 
Methodology and Applications

Gas-phase chemistry of actinides ions: a theoretical study of the reaction

$$
\text { of } \mathrm{U}^{+} \text {and } \mathrm{U}^{+2} \text { with } \mathrm{H}_{2} \mathrm{O}
$$

\section{Maria del Carmen Michelini, Nino Russo and Emilia Sicilia}

Dipartimento di Chimica and Centro di Calcolo ad Alte Prestazioni per Elaborazioni Parallele e Distribuite-Centro d' Eccellenza MURST, Università della Calabria, I87030 Arcavacata di Rende, Italy

The ability of atomic uranium cations, $\mathrm{U}^{+}$and $\mathrm{U}^{+2}$, to activate the $\mathrm{O}-\mathrm{H}$ bond of $\mathrm{H}_{2} \mathrm{O}$ is studied by using different approaches of Density Functional Theory (DFT). A close description of the reaction mechanisms leading to different reaction products is presented, including all the involved minima and transition states. At the first step of the reaction the metal cation insertion proceeds through the formation of an ion-dipole complex. In a second step, an hydrogen shift process leads to the formation of the insertion products. From these intermediates two different dissociation channels have been considered.

Recent theoretical works have reported some contrasting conclusions about the performance of GGA versions of DFT with respect to hybrid functionals for actinide-containing molecules. Therefore, three different theoretical approaches have been chosen to perform these calculations, firstly DFT in its B3LYP formulation has been used together with the Sttugart RECP (small core) for uranium atom, secondly the same RECP have been used together with the PW91 functional for exchange and correlation parts. Finally, the ZORA approximation has been used together with PW91 functionals, as implemented in ADF package. The influence of spin-orbit interaction effects has been analysed by performing Spin-Orbit ZORA calculations.

The obtained results are compared with recent experimental data, in which the reaction pathways and rate constants of gas-phase uranium ions with $\mathrm{H}_{2} \mathrm{O}$ have been investigated using a quadrupole ion trap mass spectrometer.
P111

DFT predictions of isotropic hyperfine coupling constants of the nuclei of the first, second and third rows.

\section{Hermosilla, P. Calle, C. Sieiro and J.M. Garcia de la Vega}

Departamento de Química Física Aplicada, Facultad de Ciencias, Universidad Autónoma de Madrid, 28049 Madrid, Spain

The reliability of density functional theory (DFT) in the determination of isotropic hyperfine coupling constants (hfccs) of the ground states of radicals containing ${ }^{1} \mathrm{H},{ }^{9} \mathrm{Be},{ }^{11} \mathrm{~B},{ }^{14} \mathrm{~N},{ }^{17} \mathrm{O},{ }^{19} \mathrm{~F},{ }^{23} \mathrm{Na},{ }^{25} \mathrm{Mg},{ }^{27} \mathrm{Al},{ }^{29} \mathrm{Si},{ }^{31} \mathrm{P},{ }^{33} \mathrm{~S}$ and ${ }^{35} \mathrm{Cl}$ nuclei is examined. Predictions using three hybrid functionals (B3LYP, B3P86 and B3PW91) and four different basis sets: Pople's 631G*, Dunning's cc-pVQ(T)Z, Ahlrichs's TZPV and Barone's EPR-III are made and compared to experimental values.

The DFT methodology is giving accurate values of spin densities and is allowing good assignments of experimental hfccs ${ }^{1,2}$. The use of these DFT methods allows to compute hfccs with a lower time cost, using the same basis sets with respect to other post-HF methods. We have studied 150 neutral radicals, anions and cations with doublet and quartet electronic states and we have analyzed systems from small diatomic radicals to large size, which furnish about 400 hfccs. Regression analysis is used as an appropriate methodology for thid kind of comparative study.

The most accurate theoretical predictions are obtained from B3LYP/EPR-III for protons and second row nuclei and from $\mathrm{B} 3 \mathrm{LYP} / \mathrm{ccpVQ}(\mathrm{T}) \mathrm{Z}$ for the third row of nuclei. However, the combination B3LYP/TZPV//B3LYP/6-31G* scheme results in an excellent predictor of hfccs for radicals of small, moderate and large sizes.

[1] C. J. Cramer, Essentials of Computational Chemistry. Theories and Models. Wiley: Chichester, 2002.

[2] L. Hermosilla, P. Calle, J.M. Garcia de la Vega and C. Sieiro, J. Phys Chem. A, 2005, 109, 1114; L. Hermosilla, P. Calle, and C. Sieiro, Phosphorus, Sulphur and Silicon and Related Elements (in press).
Methodology and Applications

P110

Electrostatic Control on endo/exo Selectivity in Polar Cycloaddition

$$
\text { V.Tamilmani }^{\star \#} \text {, C. A. Daul }{ }^{\star} \text {, P.Venuvanalingam }{ }^{\#}
$$

${ }^{\star}$ Department of Chemistry ,University of Fribourg, Fribourg, Switzerland \# Department of Chemistry, Bharathidasan University,Tiruchirappalli, India

2,3-Dimethylisoquinolinium cation (1) undergoes cationic Diels-Alder cycloaddition with cyclopentadiene (CP) and preferably exo adduct is formed [1]. Bradsher and coworkers have performed this reaction in acetonitrile and proposed concerted mechanism for this reaction because of the reason that the intermediate carbonium ion could not be detected during the reaction [2]. They have further argued that the exo adduct was favoured over endo adduct in view of higher electrostatic repulsion between the reacting partners in the endo approach than in the exo approach.<smiles>[N+]c1cc2ccccc2cc1[N+](=[N+])[O-]</smiles><smiles>C1=CC2CCC1C2</smiles><smiles>CC1=[N+](C)C2c3ccccc3C1C1C3C=CC(C3)C12</smiles>

exo

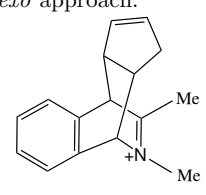

endo

The reaction has now been modeled at ab initio and DFT levels and computed results predict that the reaction is expectedly inverse electron demand type and follows concerted mechanism with extremely asynchronous transition structures. Significant amount of charge has been transferred from cyclopentadiene to cation (1) in the transition states (TS) and this introduces electrostatic repulsion between the delocalized charge in the CP and 1. This repulsion is higher in the endo TS than in the exo TS in view of spatial proximity of the charge centers in the former. The exo TS is low lying and an activation energy difference of $1.98 \mathrm{kcal} / \mathrm{mol}$ is computed at B3LYP $/ 6-31 \mathrm{G}(\mathrm{d})$ level. Though the reaction is found to be exothermic, computed barriers and reaction energies predict that the reaction is kinetically controlled. Slightly longer distance between the charge centers in the endo TS imply stronger elctrostatic repulsion and that destabilizes the endo TS, and this is in conformity with the stereoselectivity rule for polar cycloadditions.

[1] C. K. Bradsher, F. H. Day, A. T. McPhail and P. S. Wong, J. Chem. Soc. Chem. Comm, 1973, 5, 156 .

[2] C. K. Bradsher, G. L. B. Carlson, N. A. Porter and I. J. Westerman, J. Org. Chem, 1978, 43, 828 .

Methodology and Applications

Electron excitation calculations of 1,8 naphtalimides and derivatives by TD-DFT methods. Effects of substituents and solvent environment.

\section{J.L.G. Coronado ${ }^{\#}$, E. Martín ${ }^{\#}$, L.A. Montero ${ }^{\& \#}$ and J.M. García de la Vega ${ }^{\#}$}

\# Departamento de Química Física, Facultad de Ciencias, Universidad Autónoma de Madrid, 28049 Madrid, Spain.

\& Laboratorio de Química Computacional y Teórica, Facultad de Química, Universidad de la Habana, Havana 10400, Cuba.

Naphthalimides are a class of compounds with high antitumor activity upon a variety of human tumor cells. Moreover, some 1,8-naphthalimide derivatives present interesting properties as fluorescent substances and have been used as active media in tuneable dye lasers and as fluorescence markers [1]. Molecular clustering with different solvents with several dipole moment and dielectric constant and their respective UV spectra are calculated: AcH (1.7 D; 6.20), pdioxane (0 D; 2.21), $\mathrm{MeCN}(3.9 \mathrm{D} ; 36.6), \mathrm{Cl}_{2} \mathrm{CH}_{2}(1.6 \mathrm{D}, 8.93)[2,3]$. The corrections due to the molecular environmental effects in isolated 1,8 naphthalimide (see Figure) are considered by means of a complete screening of possible solute-solvent configurations. Vertical excitations are calculated with TD-DFT procedures and results are compared with other methods. Solvent shells are more realistic, insight chemistry, regarding hydrogen bonding and electrostatic interactions. The quantum chemical vertical transitions in local minima of supermolecules reveal good agreement with the experimental results on absorption spectra. An unusual shoulder in the experimental principal band is predicted with this methodology. The presented procedure seems to be reliable for the unsubstituted molecule and it is feasible to apply to other<smiles>O=C1NC(=O)c2cccc3cccc1c23</smiles>
substituted families of 1,8-naphthalimide.

The effect of substituent is studied considering different groups (-CH2-CH2-N-CH3, -O-Me, - NH2 and - NH-CO$\mathrm{Me})$ at $\mathrm{NH}$ and at 3 or 4 positions on the naphtalic ring. The introduction of electron-donor substituents provokes an emission with a high fluorescence quantum yield.

[1] W.W. Stewart, Nature, 1981, 292, 17

[2] A. Pardo et al., J. Photochem. Photobiol. A 198948259

[3] E. Martin et al., J. Lumin. 199668157. 
Methodology and Applications

\section{Modeling the Structure-Reactivity Relationship of Acrylates and Diacrylates}

Isa Degirmenci, Duygu Avc1, Viktorya Aviyente Chemistry Department, Bogazici University, 34342 Bebek, Istanbul, Turkey

Photoinitiated free radical polymerization of multifunctional monomers produces highly crosslinked networks with high thermal stability, mechanical strength and resistance to solvent absorption. These networks are widely employed in coatings for flooring and furniture, dental restorative materials, optical fiber coatings, hard and soft contact lenses, and photolithography.[1-2]<smiles>CCC(=O)C(=O)OC</smiles>

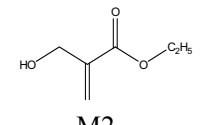

M2<smiles>CCC(=O)C(=[W])CO</smiles>

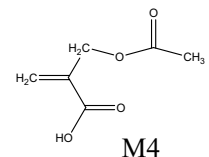<smiles>C=CC(=O)OC(=O)O</smiles>

M5
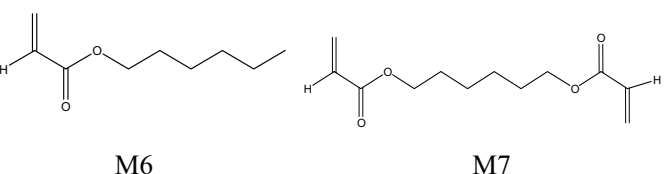

M7

In this study, the structure-reactivity relationship of monomers has been investigated with the B3LYP/6-31G* methodology. The experimentally observed polymerization behaviors have been explained based on computational findings.

[1] D. Avci, L. J. Mathias, Polymer, 2004, 45,1763.

[2] T. J. Smith, B. S. Shemper, J. S. Nobles, A. M. Casanova, C. Ott, L. J. Mathias, Polymer, 2003,44,6211.

Methodology and Applications

P115

Relative stabilities of zeotype materials from DFT Calculations: Some effects of structure and stoichiometry.

Kathrine Mejland, ${ }^{\dagger}$ Karl Petter Lillerud, ${ }^{\dagger}$ and $\underline{\text { Ole Swang }}{ }^{\ddagger}$

†:Dept. of Chemistry, U. of Oslo, P. O. B. 1030 Blindern, N-0315 Oslo, Norway, and $\ddagger$ :Dept. of Hydrocarbon Process Chemistry, SINTEF Materials and Chemistry, P. O. Box 124 Blindern, N-0314 Oslo, Norway

Zeolites are an important class of materials due to their unique combination of crystallinity and microporosity. The tetrahedrally coordinated atoms (Tatoms), combined with the flexibility of the oxygen linkage between them, gives an extremely large number of possible topologies. We have investigated the relative stabilities of a large number of pure-silica zeolites using band structure DFT calculations. The results allow analyses of the relationship between structure and stability. Further, systems containing other elements in the $\mathrm{T}$ positions have been investigated using both periodic and cluster models. We discuss the influence of different non-silicon Tatoms on the stability of different topologies.

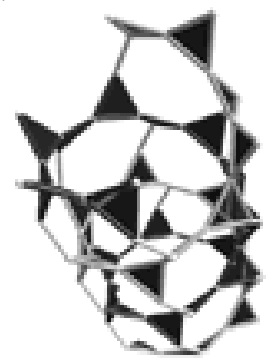

[1]Van Bekkum, H.; Flanigen, E. M.; Jansen, J. C., Eds., Stud. Surf. Sci. Catal. 1991, 58; http://www.iza-online.org
Methodology and Applications

An ab initio Investigation of the DNA Photolyase Repair Activity

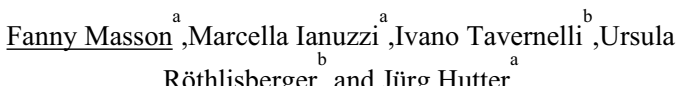
Röthlisberger and Jürg Hutter

Institute of Physical Chemistry, University of Zurich, CH-8057 Zurich, Switzerland

Laboratory of Computational Chemistry and Biochemistry, Federal Institute of Technology (EPFL), CH-1015 Lausanne, Switzerland

The most common type of DNA damage caused by UV radiation is the $[2+2]$ cycloaddition of two adjacent pyrimidine bases which yields cyclobutane pyrimidine dimers (CPDs) [1]. Kinetic studies suggested that the formation of the photodimer arises from the reaction of one molecule in a triplet state with a second one in its ground state [2] DNA photolyases use light energy to repair the CPD lesions by transferring an electron to the cyclobutane ring. The resulting radical anion then splits into two pyrimidines and transfers back the excess electron to the enzyme. However the atomistic details of the reaction mechanism that transforms the dimmer anion into a monomer and a monomer radical anion are still not well understood. The purpose of our work is to shed light on the details of the dimmer formation and dissociation process, first in vacuo and then in its natural environment, i.e. within DNA. We address this issue by using a hybrid Car-Parrinello quantum mechanical/molecular mechanical (QM/MM) approach, in which the complex DNA environment is treated with a classical force field and the ground state potential energy surface and the excitation energies of the dimmer are described within density functional theory (DFT) and time dependent (TD) DFT, respectively.

[1] A. Sancar, Chem. Rev., 2003, 103 ,2203-2237.

[2] E. Sztumpf-Kulikowska, Photochem. Photobiol., 1967, 6, 41.

Methodology and Applications

P116

Assessment of theoretical prediction of the NMR shielding tensor of

${ }^{195} \mathrm{PtCl}_{\mathrm{x}} \mathrm{Br}_{6-\mathrm{x}}{ }^{2-}$ complexes by DFT calculations: experimental and computational results.

\section{Peter Belser ${ }^{1}$, Henry Chermette ${ }^{2}$, Claude Daul ${ }^{1}$, Emmanuel Penka}

${ }^{1}$ Département de Chimie, Université de Fribourg, chemin du Musée 9, CH1700 Fribourg

${ }^{2}$ Laboratoire de Chimie Physique Théorique, bât. 210, Université Claude Bernard Lyon-1 and UMR CNRS 5182, 43 bd du 11 novembre 1918, 69622Villeurbanne cedex, France.

In this communication, the ZORA spin-orbit Hamiltonian is used in conjunction with the gauge including orbital (GIAO) method based on DFT theory to calculate ${ }^{195} \mathrm{Pt}$ chemical shift of ${ }^{195} \mathrm{PtCl}_{\mathrm{x}} \mathrm{Br}_{6-\mathrm{x}}{ }^{2-}$ complexes. Excellent agreement with experiments has been obtained for calculations bearing on optimized geometries and all electrons triple zeta + polarization (TZP) STO basis sets. The relative error with respect to experiment is less than $1.5 \%$. It is found that the Pt chemical shift is dominated by the paramagnetic and the spin orbit contribution, whereas the diamagnetic term remains negligible. The influence of the quality of the basis sets has been studied and found small provided a basis set like TZP is used. Several calculations have been performed in order to establish the sensitivity of the chemical shift to a variation in the bond lengths. A strong dependence has been found, with an increase of the chemical shift amounting to $150 \mathrm{ppm} / \mathrm{pm}$ for a distance decrease. A large sensitivity upon solvation is then expected, leading to substantial structural changes. Different tests using conductor like screening models have been performed in order to establish the sensitivity of the chemical shift to solvation. It has been observed that the changes in the geometry are more important than charge transfers. Finally the sensitivity of the system to the exchange-correlation functional is found rather weak, at least among the GGA functionals. 
Methodology and Applications

Aromaticity in tellurium heterocycles: Is $\sigma$ bonding significant in Hückel-type rings?

Nuno A. G. Bandeira (nuno.bandeira@,fc.ul.pt) and Maria José Calhorda (mjc@fc.ul.pt)

Department of Chemistry and Biochemistry,

Faculty of Science, University of Lisbon - C8 building, Campo Grande, 1749-016 Lisbon, Portugal

The synthesis of the $\mathrm{Te}_{2} \mathrm{~N}_{2} \mathrm{SCl}_{2}$ heterocycle (3,3-dichloro-1-sulfi-2,5-diazi-3,4ditelluri-[5]cycle) and its derivative cation $\left[\mathrm{Te}_{2} \mathrm{~N}_{2} \mathrm{SCl}^{+}\right.$by Haas and Pryka ${ }^{[1], 2]}$ prompted research into the Hückel type aromaticity exhibited in the $\left[\mathrm{Te}_{2} \mathrm{~N}_{2} \mathrm{~S}\right]^{2+} 6 \pi$ dication (Figure 1) and how ring currents vary as a function of the number of chloride atoms added to the tellurium in the ring.

Geometry optimisations (Figure 2), NBO analyses ${ }^{[3]}$, calculation of $\mathrm{NICS}^{[4]}$ and ring current shieldings ${ }^{[5]}$ were performed and the results will be presented.

The NICS value for the neutral species $\mathrm{Te}_{2} \mathrm{~N}_{2} \mathrm{Cl}_{2}$ (Figure 2, left) is over $-14 \mathrm{ppm}$, which suggests that the presence of two chlorine groups and the change in formal oxidation state from $\mathrm{Te}$ (II) to $\mathrm{Te}(\mathrm{IV})$ is not enough significant to disrupt the diatropic ring current.

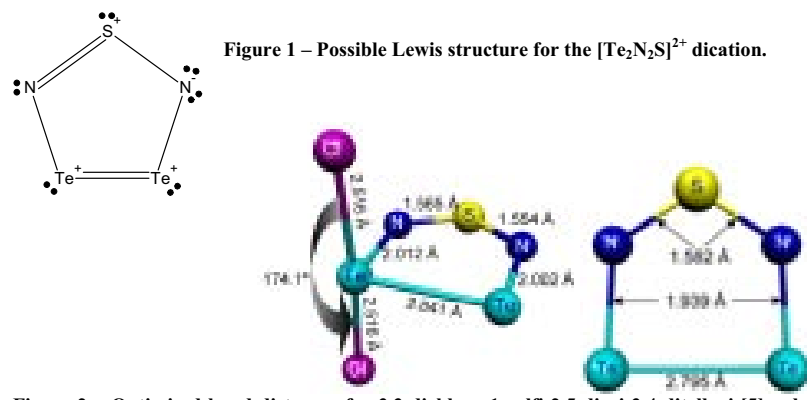

Figure 2 - Optimised bond distances for 3,3-dichloro-1-sulfi-2,5-diazi-3,4-ditelluri-[5]cycle and 1-sulfi-2,5-diazi-3,4-ditelluri-[5]cyclium(2+) respectively. The latter is Hückel aromatic.

[1] A. Haas, M. Schaefers, M. Pryka, Chem. Ber. 1994, 127, 1865.
[2] Haas, M. Pryka, J. Chem. Soc., Chem. Comm. 1994, 391.

[3] E. D. Glendening, J. K. Badenhoop, A. E. Reed, J. E. Carpenter, J. A. Bohmann, C. M.

Morales, F. Weinhold, NBO 5.0 ed., 2001

[4] P. R. Schleyer, C. Maerker, A. Dransfeld, H. Jiao, N. J. R. E. Hommes, J. Am. Chem. Soc

1996, 118,6317 .

J. Jusélius, D. Sundholm, Phys. Chem. Chem. Phys. 1999, 1, 3429.

Methodology and Applications

P119

Application of Density Functional Theory Calculation to Lanthanide and Actinide Complexes

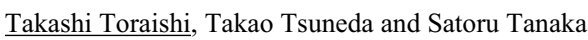

Department of Quantum Engineering and System Science, Graduate School of Engineering, The University of Tokyo, 7-3-1 Hongo, Bunkyo-ku, 113-8656 Tokyo, Japan.

Density Functional Theory (DFT) calculation now becomes a widely-used technique for the chemistry of lanthanide and actinide complexes due to its low calculation cost and high accuracy. However, very few works on the charge transfer (CT) phenomena of $f$-elements was indeed reported, although $\mathrm{CT}$ is one of key reactions of $f$-elements chemistry in several application fields, e.g. biological sensing, optical device development and nuclear engineering. The reason is simple: conventional DFTs give poor results for CT reactions. However, this argument is no longer correct. The choice of proper functional and basis sets allows us to discuss CT phenomena quantitatively.

On this ground, we examined two CT reactions as trial systems, and demonstrate the applicability of DFT to the CT reaction of $f$-block elements. First, the ligand-to-metal charge transfer (LMCT) in photoluminescence of trivalent lanthanides- 5-sulfosalicylate complex will be presented. LMCT by photoexcitation was successfully treated by time-dependent DFT method, and we firstly found that CT takes place via mixing of $4 f$ orbitals with $\pi^{*}$ orbitals in the vertically excited lowest triplet state. ${ }^{1}$ Second, we will present the reduction of $\mathrm{U}(\mathrm{VI})$ by $\mathrm{Fe}(\mathrm{II})$. We found that the pure density functional is not suitable for calculating redox reactions, since the long-range correlation strongly affects CT reactions. The long-range correction scheme ${ }^{2}$ is thus applied and we will discuss a new DFT method for investigating charge transfer reactions of 5 -elements.

[1] T. Toraishi, M. Asakura, T. Tsuneda and S. Tanaka, submitted.

[2] H. Iikura, T. Tsuneda, T. Yanai and K. Hirao, J. Chem. Phys., 2001, 115, 3450 .
Methodology and Applications

P118

Interpretation Of Hydrogen Bonding In The Weak And Strong Regions Using Conceptual DFT Descriptors

\section{$\underline{\text { Alimet Sema Özen }^{1}}$, Viktorya Aviyente ${ }^{2}$, Frank De Proft ${ }^{3}$, Paul Geerlings ${ }^{3}$}

Faculty of Engineering and Natural Sciences, Sabanci University 34956 Istanbul, Turkey ${ }^{1}$

Department of Chemistry, Bogazici University 34342 Istanbul, Turkey ${ }^{2}$ Faculty of Sciences, Department of General Chemistry, Free University of Brussels 1050 Brussels, Belgium ${ }^{3}$

Hydrogen bonding is a unique type of molecular interactions not only for its fundamental role in the vital biological and chemical processes, but also for the amount of ambiguity in its interpretation as a "weak" interaction. In reality, the spectrum of hydrogen bonds extends from $1-4 \mathrm{kcal} / \mathrm{mol}$ for weak bonds to $4-15 \mathrm{kcal} / \mathrm{mol}$ for moderate bonds and $15-40 \mathrm{kcal} / \mathrm{mol}$ for strong bonds. ${ }^{1}$ In a review paper as a part of their systematical and beautiful work on hydrogen bonds, Gilli et al. calls the lack of general chemical rules or a unified hydrogen-bond theory as $H$-bond puzzle. ${ }^{2}$ The aim of the present study is to get only an alternative view on solving this puzzle using the tools of conceptual DFT. As a part of this task, high and uniform level calculations on hydrogen bond energy and related quantities such as PA, $\mathrm{pK}_{\mathrm{a}}$ and DFT reactivity descriptors have been presented altogether here for the first time.

[1] Jeffrey, G.A.; An Introduction to Hydrogen Bonding, Oxford University Press, New York, 1997.

[2] Gilli, G.; Gilli, P; J.Mol. Struc. 2000,552,1-15.

Methodology and Applications

P120

Fulvenes, Arylolefins, and Baird's Theory on Triplet State Aromaticity

Raül Crespo, ${ }^{\ddagger}$ Mari Carmen Piqueras, ${ }^{\star}$ Haruhisa Kato $^{\dagger}$ and $\underline{\text { Henrik Ottosson }}{ }^{\dagger}$

* Departament de Química Física, Universitat de València, Dr. Moliner 50, E-46100 Burjassot, Spain, and ${ }^{\dagger}$ Department of Chemistry, Uppsala University, Box 599, SE-751 24 Uppsala, Sweden

In 1972, Baird derived using PMO-theory that annulenes which are aromatic in their singlet ground states $\left(\mathrm{S}_{0}\right)$, are antiaromatic in their lowest triplet states $\left(\mathrm{T}_{1}\right)$, and vice versa. ${ }^{1}$ This theory can be used to show that fulvenes act as aromatic chameleons as they polarize the electron density distribution so as to adopt some aromatic character in both $S_{0}$ and $T_{1}$ (Scheme 1). ${ }^{2}$ This gives opposing polarities of fulvenes in $S_{0}$ and $T_{1}$, and their singlet-triplet energy gaps $\left(\Delta E_{\mathrm{ST}}\right)$ can be tuned through substitution. Using computations, it is even possible to design fulvenes with triplet ground states. A thorough comparison of results from OLYP density functional theory calculations were made with results from CASSCF and CASPT2. This includes $\Delta E_{\mathrm{ST}}$, geometries, dipole moments, and aromaticity probes. Baird's theory can also be used to show that the shape of the $\mathrm{T}_{1}$ state potential energy surface of arylolefins is strongly influenced by aromaticity and antiaromaticity (Scheme 2 ). Again, we compared $\mathrm{T}_{1}$ state properties from OLYP calculations with those from CASSCF and CASPT2.

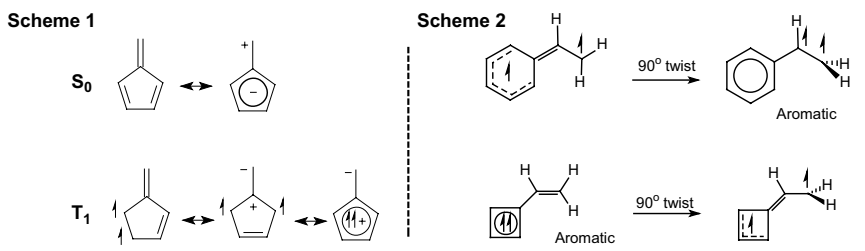

[1] Baird, N. C. J. Am. Chem. Soc. 1972, 94, 4941.

[2] Möllerstedt, H., Piqueras, M. C., Crespo, R. Ottosson, H. J. Am. Chem. Soc. 2004, 126, 13938. 
Methodology and Applications

The adsorption of NTCDA on the $\operatorname{Ag}(110)$ surface: insights from GGA density functional calculations

Audrius Alkauskas, Alexis Baratoff, and Christoph Bruder

Institute of Physics, University of Basel

Klingelbergstrasse 82, CH-4056 Basel, Switzerland

Inspired by the experimental work of Fink et al. [1] we have studied the adsorption of 1,4,5,8-naphthalene tetracarboxylic dyanhydride (NTCDA), a strong electron acceptor, on the $\mathrm{Ag}(110)$ surface by means of density functional theory calculations. The ABINIT plane wave DFT code is employed in our work. We find that the PBE-GGA functional is rather successful and predicts the correct local adsorption geometry. Among the four tested local adsorption sites, the most stable, top site, is characterized by an adsorption energy of $\sim 0.9 \mathrm{eV}$, close to the value deduced from experimental measurements on $\mathrm{Ag}(001)$ [1]. The potential energy surface describing the molecule-substrate interaction is rather shallow and more than 100 geometry optimization steps are needed to converge the residual forces. Like in the case of PTCDA on the $\mathrm{Ag}(111)$ surface [2], the carbonyl oxygens are $\sim 0.15 \AA$ closer to the underlying substrate than the aromatic backbone. The binding of NTCDA to the $\mathrm{Ag}(110)$ surface is rationalized by 1) charge transfer from the substrate to the $\pi$-type LUMO of NTCDA ( $\sim 0.6$ electrons) and 2) electrostatic attraction between negatively charged carbonyl oxygens and positively charged silver atoms which is enhanced when those oxygens are close to $\mathrm{Ag}$ close-packed $[1 \overline{1} 0]$ rows. Concerning the lateral packing of molecules, DFT predicts essentially the same adsorption energy in the $(30 / 03)$ and experimentally oberved $(30 / 13)$ superstructures, showing that the stabilization of the latter occurs owing to weak van-der-Waals interactions not grasped by the semilocal implementations of DFT. Conclusions regarding the application of our results to related organic-on-metal systems [3] are drawn.

[1] R. Fink et al., Phys. Rev. B, 1999, 60, 2818

[2] A. Hauschild et al., Phys. Rev. Lett., 2005, 94, 036106.

[3] M. Eremtchenko et al., Nature, 2003, 425, 602.

Methodology and Applications

P123

\section{Application of PDFT to scale the strength of Brønsted acid sites} in different zeolites

Mohamed Elanany and Daniel P. Vercauteren

Département de Chimie, University of Namur, Rue de Bruxelles 61, B-5000 Namur, Belgium.

Crystalline microporous molecular sieves (MMS) are used to a large extent as catalysts in oil refining and petrochemical industries, their acidic properties controlling mainly the catalytic activities [1]. With the continuous increase of computational power, theoretical methods have become significant tools for characterizing the acidic properties of these MMS [2]. Parrillo and co-workers [3,4] showed experimentally that the relation between the adsorption energy of some bases and their proton affinities in the gas phase is interesting and that is important to scale the acidity of different MMS. Hence, in this study this relation is investigated using computational methods viz., density functional theory (DFT), employing periodic zeolite models $(\sim 150$ ions/uc) in order to obtain reliable, realistic, and accurate results. Table 1 shows that energy calculations by GGA, taking into account zero point energy, give very good Calculated Reported agreement with the reported for proton affinity in the gas phase.

Figure 1 shows the calculated adsorption energies of these bases on a Brønsted acid at $\mathrm{T}_{4}$ site in mordenite crystal (black dots) versus the proposed line with slope of unity. It can be deduced that primary amines fit much better than sec- or tert-amines. Therefore primary amines should be used as probe molecules when we try to scale the acidity of zeolites on the basis of this relation.

[1] Weitkamp, J. Solid State Ionics 2000, 131,175

[2] Brändle, M.; Sauer, J. J. Am. Chem Soc. 1998, 120, 1556 .

[3] Parrillo, D. J.; Gorte, R. J.; Farneth, W. J. Am. Chem. Soc. 1993, 115, 12441 .

\begin{tabular}{lcc} 
& $\begin{array}{c}\text { Calculated } \\
\text { proton affinity in } \\
\text { the gas phase } \\
\text { (kJ/mol) }\end{array}$ & $\begin{array}{c}\text { Reported } \\
\text { values } \\
(\mathbf{k J} / \mathbf{m o l})[\mathbf{3}]\end{array}$ \\
\hline ammonia & 853.4 & 858.3 \\
methylamine & 897.4 & 896.4 \\
ethylamine & 904.3 & 909.0 \\
isopropylamine & 923.4 & 918.6 \\
dimethylamine & 927.6 & 923.2 \\
trimethylamine & 932.4 & 939.1 \\
\hline
\end{tabular}

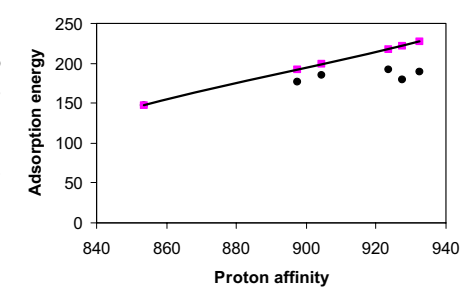

[4] Lee, C.; Parrillo, D. J.; Gorte, R. J.; Farneth, W. E. J. Am. Chem. Soc. 1996, 118, 3262
Methodology and Applications

P122

\section{Electronic feature and acidity of M-AIPOs: a PDFT study}

Mohamed Elanany and Daniel P. Vercauteren

Département de Chimie, University of Namur, Rue de Bruxelles 61, B-5000 Namur, Belgium.

Many attempts, using different syntheses methods, have been devoted to incorporate isomorphously metal ions in alumonophosphate (AIPO) frameworks to get novel acidic or redox properties [1]. Our previous results using periodic density functional theory (PDFT) method [2] showed very good correlation between Brønsted acidity theory (PDFT) method [2] showed very good correlation between Brønsted acidity and electronegativity of the incorporated elements $(\mathrm{Al}, \mathrm{B}$, and $\mathrm{Ga})$ in chabazite.
However in the case of transition metal ions in AlPOs, rationalization for the strength of Brønsted acidity is still far from understanding $[2,3]$. In this study the relation between Brønsted acidity of $\mathrm{M}^{\mathrm{II}}-\mathrm{AlPO} 34(\mathrm{M}=\mathrm{Cr}, \mathrm{Mn}, \mathrm{Fe}, \mathrm{Co}$ or $\mathrm{Ni})$ and the electronic states of the dopants (Figure a) is investigated using DFT employing periodic models. Geometry optimizations and energy calculations were performed using $\mathrm{Dmol}^{3}$ [4], while CASTEP code [5] has been used for DOS calculations. The calculated adsorption energies of $\mathrm{NH}_{3}$, as a basic probe, for $\mathrm{Mn}, \mathrm{Co}, \mathrm{Cr}, \mathrm{Ni}$, and $\mathrm{Fe}-$ AlPO34 are -136.1, -128.9, -94.6,-118.4, and $-124.3 \mathrm{~kJ} / \mathrm{mol}$, respectively. This AlPO34 are $-136.1,-128.9,-94.6,-118.4$, and $-124.3 \mathrm{~kJ} / \mathrm{mol}$, respectively. This
indicates the strong Brønsted acidity of Mn-AlPO34. Figures b shows partial DOS indicates the strong Brønsted acidity of Mn-AlPO34. Figures b shows partial DOS
analyses of MnAlPO-34. For clarity the total DOS was scaled down by four. In the case of Mn-AlPO34, $3 d$ orbitals are equally occupied in the high spin state; consequently the four $\mathrm{O}$ ions around $\mathrm{Mn}$ are exposed to a uniform repulsion, which decreases the ionic character of $\mathrm{Mn}-\mathrm{O}$ bond compared to e.g., $\mathrm{Co}-\mathrm{O}, \mathrm{Fe}-\mathrm{O}, \mathrm{Cr}-\mathrm{O}$ bonds. More details will be given in the presentation.
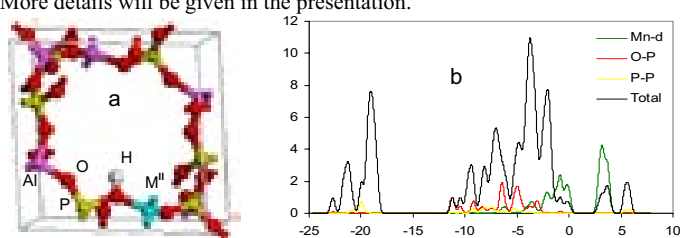

[1] Weckhuysen, B.M.; Rao, R. R.; Martens, J. A.; Schoonheydt, R.A. Eur. J. Inorg. Chem. 1999, 565.

[2] Elanany, M.; Koyama, M.; Kubo, M.; Selvam, P.; Miyamoto, A. Micropor. Mesopor. Mater. 2004, 71, 51.

[3] Cora, F.; Catlow, C. R. A.; Civalleri, B.; Orlando, R. J. Phys. Chem. B. 2003, 107, 11866

[4] Delley, B. J. Chem. Phys. 1990, 92, 508

[5] Segall, M. D.; Lindan, P. J. D.; Probert, M. J.; Pickard, C. J.; Hasnip, P. J.; Clark, S. J.; Payne, M.C. J. Phys.: Condens. Matter 2002, 14, 2717.

Methodology and Applications

P124

\section{Theoretical study of retinal analogues: \\ QM/MM and quantum chemical approach}

$\underline{\text { Minoru Sugihara }}^{1}$, Julia Hufen $^{2}$ and Volker Buss ${ }^{2}$

\author{
Theoretical Low Temperature Physics, ${ }^{1}$ Theoretical Chemistry ${ }^{2}$ \\ University of Duisburg-Essen \\ 47048 Duisburg, Germany
}

Rhodopsin is the photoreceptor for dim light vision in the rod cells of the vertebrate eyes. The chromophore of rhodopsin is 11-cis-retinal protonated Schiff base (I) which is connected to opsin through Lys296. Based on the crystal structures, ${ }^{1,2}$ we have modelled the rhodopsin chromophore ${ }^{2}$. In this study, we present theoretical investigations of three retinal analogues: 13demethyl (2), 10-methyl,13-demethyl (3), and 10-methyl (4) retinal protonated Schiff bases. Modelling work of the analogues are performed by using a combined quantum mechanical / molecular mechanical (QM/MM) method assuming the native protein and the optical properties are calculated by Molcas program package based on the reduced ( 5 double bond) systems. ${ }^{3}$ The presence of the methyl-group(s) enhances the torsion about the $\mathrm{C} 11 \mathrm{C} 12$ and $\mathrm{C} 12 \mathrm{C} 13$ bonds. All analogues show the same chiral discrimination in the binding pocket, i.e. negative and positive chiralities for the $\mathrm{C} 11 \mathrm{C} 12$ and

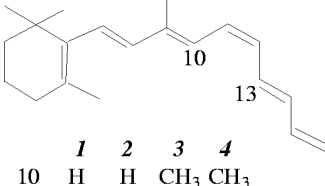

$\begin{array}{lllll}10 & \mathrm{H} & \mathrm{H} & \mathrm{CH}_{3} & \mathrm{CH}_{3} \\ 13 & \mathrm{CH} & \mathrm{H} & \mathrm{H} & \mathrm{CH}_{3}\end{array}$ $\mathrm{C} 12 \mathrm{C} 13$ bonds, respectively. The dihedral angles are $-18,-15,-23$, and $-27^{\circ}$ for the $\mathrm{C} 11 \mathrm{C} 12$ bonds and 172 , 179,174 and $165^{\circ}$ for the $\mathrm{C} 12 \mathrm{C} 13$ bonds. The calculated circular $\mathrm{NH}^{+}$dichroism intensities are are: 0.66, $0.33,0.82$, and 0.55 a.u. $^{4}$ (in the

Lys296 order of the numbering).

[1] T. Okada, Y. Fujiyoshi et al. Proc. Natl. Acad. UAS 2002, 99, 5982.

[2] T. Okada, M. Sugihara et al. J. Mol. Biol. 2004, 342, 571.

[3] J. Hufen, M. Sugihara, V. Buss, J. Phys. Chem. B 2004, 108, 20418.

[4] M. Sugihara, J. Hufen, V. Buss, submitted to Biochemistry. 
Methodology and Applications

\section{DFT Method Estimation of Standard Redox Potential of Metals and Organic compounds}

\section{Yoshiki Shimodaira $^{1}$, Akihiko Kudo ${ }^{1}$ and Hisayoshi Kobayashi ${ }^{2}$}

${ }^{1}$ Department of Applied Chemistry, Faculty of Science, Tokyo University of Science, 1-3 Kagurazaka, Shinjuku-ku Tokyo 162-8601

${ }^{2}$ Department of Chemistry and Bioscience, Kurashiki University of Science and the Arts, 2640 Nishinoura, Tsurajima, Kurashiki 712-8505

The DFT method calculation was carried out to evaluate standard redox potential for metal/metal cation systems and organic oxidant/reductant systems. ${ }^{1}$ With the Born-Haber cycle, standard redox potential of metal/metal cation systems is composed of the cohesive energy, ionization energy, and hydration energy. Utilizing experimental values of the cohesive energy of

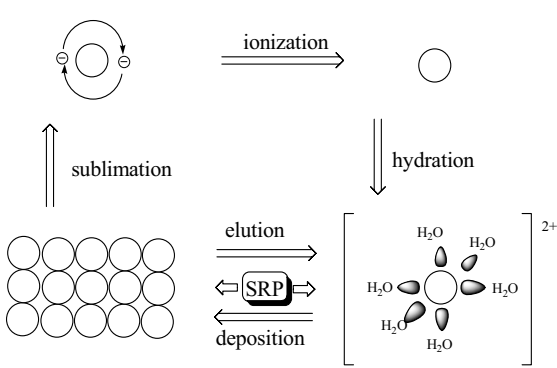
metals,

root-mean-square deviation of calculated and experimental values was $0.29 \mathrm{~V}$ for 17 ions The standard redox potential of organic oxidant/reductant systems is composed of the difference of each hydration energies. In this calculation, the solvent effect was accounted by the self consistent reaction field method, and the calculation was carried out at the level of polarized continuum model (PCM). The calculations using aqueous complex in vacuum led to the results far from the experimental values.

[1] Hisayoshi Kobayashi, et al., Chem. Lett., 2004, 33(9), 1176

Methodology and Applications

P127

DFT study of the interaction of organic molecules with gold clusters

$\underline{\text { Stanislav Záliš }}{ }^{1}$, Irena Kratochvilová ${ }^{2}$, Jakub Šebera $^{1}$ and Zdeněk Samec ${ }^{1}$

${ }^{1}$ J. Heyrovský Institute of Physical Chemistry, Academy of Sciences of the Czech Republic, Dolejškova 3, CZ-182 23 Prague, Czech Republic

${ }^{2}$ Institute of Physics, Academy of Sciences of the Czech Republic, Na Slovance 2, 18221 Praha 8, Czech Republic

The understanding of the non - linear properties of nanowires bonded to gold tips as well as catalytic properties of gold surfaces require the proper modeling the interface. We modeled gold clusters firstly as an interface simplification and secondly as a part of real experimental systems. Scalar relativistic effects were described either by relativistic pseudopotentials and corresponding wave functions or by ZORA approach. Planar and three dimensional gold clusters up to $\mathrm{Au}_{35}$ were studied.

In order to explain the experimentally obtained characteristics of negative differential resistance (NDR) we extended the calculations examining $\mathrm{Au}_{\mathrm{n}}$ bonded -4-[[2-nitro-4-(phenylethynyl) phenyl] ethynyl] benzenthiol by the influence of the static electric field and charge variation. The comparative study with molecules which do not exhibit the negative differential resistance helps with the understanding of nonlinear properties.

Cluster approach was used for the modeling of electrocatalytical epoxidation at metal interface. We studied the mechanisms of the $\mathrm{O}$ and $\mathrm{C}_{2} \mathrm{H}_{4}$ adsorption at the gold clusters of varying size and the formation of $\mathrm{Au}_{\mathrm{x}}-\mathrm{Et}-\mathrm{O}$ intermediate and the possible intermediate transfer under the influence of electric field or electrode reaction. The influence of the solvent was also modeled.

This work has been supported by the Grant Agency of the Academy of Sciences of the Czech Republic (grant No. T400400413
Methodology and Applications

The DFT Modeling of Spectral and Photochemical Properties of Diimine Transition Metal Carbonyl Complexes.

$\underline{\text { Stanislav Záliš }}{ }^{1}$, Jakub Šebera ${ }^{1}$ and Antonín Vlček, Jr. ${ }^{1,2}$

${ }^{1}$ J. Heyrovský Institute of Physical Chemistry, Academy of Sciences of the Czech Republic, Dolejškova 3, CZ-182 23 Prague, Czech Republic

2 Department of Chemistry, Queen Mary, University of London, Mile End Road, London E1 4NS, United Kingdom

The flexibility of the coordination sphere of mixed valence carbonyl complexes enables to tune spectral and protochemical properties. The variation of excited state characteristics is ilustrated on $\mathrm{Ru}, \mathrm{Re}$ and $\mathrm{W}$ mixed ligand diimine carbonyl complexes. The lowest excited states of [W(CO) $\left.{ }_{4} \mathrm{~L}\right]$ and $\left[\mathrm{W}(\mathrm{CO})_{5} \mathrm{~L}^{\prime}\right]\left(\mathrm{L}=N, N^{\prime}\right.$-di-methyl-1,4-diazabutadiene, ethylenediamine, 2,2'-bipyridine, phenantroline; $\mathrm{L}$ '= pyridine, 4-CN-pyridine, piperidine) complexes were found to be either $\mathrm{W} \rightarrow \pi^{*}(\mathrm{~L})$ or $\mathrm{W} \rightarrow \pi^{*}(\mathrm{CO})$ in character, depending on the type of the hetero-ligand. Within $\operatorname{trans}(X, X)$ $\left[\mathrm{Ru}(\mathrm{X})_{2}(\mathrm{CO})_{2}(\mathrm{~L})\right](\mathrm{X}=\mathrm{Cl}, \mathrm{Br}, \mathrm{I})$ and $f a c-\left[\operatorname{Re}(\mathrm{X})(\mathrm{CO})_{3}(\mathrm{~L})\right](\mathrm{X}=\mathrm{NCS}$, halide) complexes the lowest electronic transitions are identified as mixed $\mathrm{X} \rightarrow \mathrm{L} / \mathrm{M} \rightarrow \mathrm{L}$ ligand to ligand and metal to ligand charge transfer (XLCT/MLCT).In order to characterize how the particular lowest excited state reflects the coordination sphere variation, a combined experimental and theoretical study was performed. The interpretation and modeling of experimentally measured UV-VIS, emission, picosecond time-resolved IR and resonance Raman (rR) spectra is based on DFT calculations.

The spectral transitions and charge density redistributions in the course of excitations were calculated by the time-dependent DFT (TD-DFT) method. Lowest excited states of the closed-shell complexes were optimized at TDDFT level or unrestricted Kohn - Sham calculations on the lowest-lying triplet state. The electrostatic solvent influence on spectra was modeled using the polarizable - continuum model (CPCM) or conductor - like screening model (COSMO). Simulated ground and excited-state IR spectra correspond closely to the experimental spectra measured by ps-timeresolved IR spectroscopy.

Methodology and Applications

P128

Electrochemical properties of ferrocenyl ligands designed for ion recognition in solution

H. Chermette $^{1}$, C. Lepetit $^{2}$, B. Delavaux-Nicot ${ }^{2}$

Lab. Chimie Physique Théorique ${ }^{1}$, bât 210, Université Claude Bernard

Lyon-1 et UMR CNRS 5182, 43 bd du 11 novembre 1918, 69622Villeurbanne cedex, France.chermet@in2p3.fr

Laboratoire de Chimie de Coordination ${ }^{2}$, UPR 8241 CNRS, 205 Route de

Narbonne, 31077 Toulouse cedex 04, France.lepetit@lcc-toulouse.fr

Ferrocenyl ligands (see Figure below) have been synthesized and characterized by B. Delavaux-Nicot et al [1] in order to be used as ligands for ion recognition in solution by electrochemistry and/or fluorescence. DFT studies have been performed in order to determine electronic factors responsible of their electrochemical behaviour depending on the nature and the number of substituents of the ferrocene.

The first vertical ionization, calculated as a $\triangle \mathrm{SCF}$ difference of total energies of the ionized molecule and the neutral molecule do not correlate well to the experimental oxidation potentials $\mathrm{E}^{\circ}\left(\mathrm{Fe}^{\mathrm{II}} / \mathrm{Fe}^{\mathrm{III}}\right)$. A strong electronic reorganization of the molecule during or after the ionization process is evidenced through the strong variation of the metal character of some of the frontier orbitals.

Accordingly, the mono-ionization and di-ionization vertical potentials have been calculated. The convolution of the successive (discrete) ionization potentials weighted by either the metal character or the $\pi$ character of the molecular orbitals depleted by the ionization allows an excellent modelling of the experimental voltammograms.

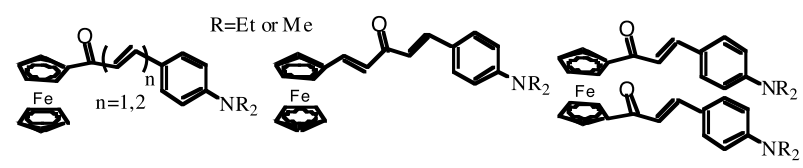

[1] B. Delavaux-Nicot, S. Fery-Forgues Eur. J. Inorg. Chem.,1999, 1821; J. Photochem. and Photobiol. A: Chemistry, 2000, 132, 137. 


\section{Alkanes Metathesis on silica supported tantalum hydride: a DFT study}

\section{S. Schinzel ${ }^{1}, \underline{\text { H. Chermette }}{ }^{1}$ C. Copéret ${ }^{2}$, J. M. Basset ${ }^{2}$}

Lab. Chimie Physique Théorique ${ }^{1}$, bât 210, Université Claude Bernard Lyon-1 et UMR CNRS 5182, 43 bd du 11 novembre 1918, 69622-Villeurbanne Cedex, France. henry.chermette@univ-lyon1.fr Laboratoire de Chimie Organométallique de Surfaces ${ }^{2}$, Bat. 308, CPE Lyon, UMR 9986 (CNRS \pm CPE Lyon), 43, boulevard du 11 novembre 1918, 69616 Villeurbanne Cedex, France.coperet@cpe.fr

The metathesis of alkanes transforms at low temperatures a given alkane into its higher and lower homologues.[1] Through a study of initiation products and higher homologue selectivities, it was recently shown that olefins and metallocarbenes were involved.[2] The detailed understanding of the key elementary steps are theoretically studied by DFT methods.

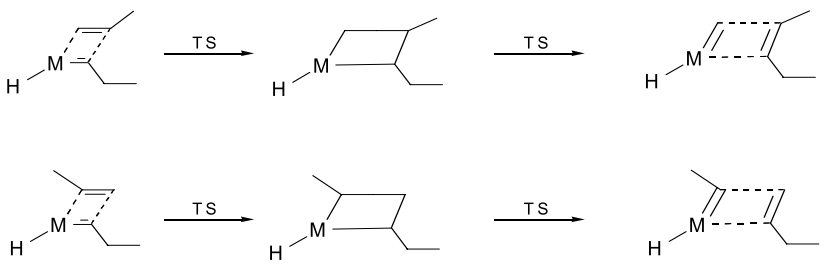

[1] V. Vidal et al., Science 1997, 276, 99.

[2] E. Le Roux et al., J. Am. Chem. Soc., 2004, 126, 13391

Methodology and Applications

Ab initio excited state MD study of photochemical processes in molecules

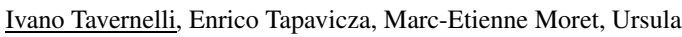
Rothlisberger

Ecole Polytechnique Fédérale de Lausanne (EPFL), Laboratoire de Chemie et Biochemie Computationelle, CH-1015 Lausanne, Switzerland

Photochemical processes are ubiquitous in chemistry and biology. Recently, the study of excited state structure and dynamics of large molecules was made possible by the development of time-dependent DFT (TDDFT) [1]. Our computational approach is based on the implementation of TDDFT in the ab initio MD code CPMD and allows the inclusion of solvation effects via a QM/MM coupling [2] with a classically described environment. Non-adiabatic effects are treated using the Landau-Zener approach [3].

In this work we investigate the orbital and nuclear rearrangements that follow the electronic vertical photo-excitation in two model reactions, (i) the intramolecular electron transfer (ET) process in an organic donor-bridge-acceptor (DBA) compound, and (ii) the metal to ligand charge transfer (MTLCT) transition in the aqueous metal complex Ruthenium(II) tris-2,2'bipyridine.

In this last example, we show ev-

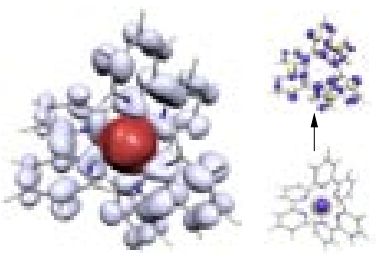
idences for a triplet state charge

localization on two ligands, which occurs simultaneously with a $D_{3}$ to $C_{2}$ symmetry lowering.

[1] E. Runge and E.K.U. Gross, Rhys. Rev. Lett., 1984, 52, 997 J. Hutter, J. Chem. Phys., 2003, 118, 3928.

[2] A. Laio, J. VandeVondele and U. Rothlisberger, J. Chem. Phys. 2002, 116, 6941

[3] C. Zener, Proc. Roy. Soc. London, 1934, A 145, 523.
Structural, Electronic and Optical Properties of Surface saturated Cadmium Sulfide Clusters

$\underline{\text { Johannes Frenzel }}^{1}$, Gotthard Seifert ${ }^{1}$, Jan-Ole Joswig ${ }^{2}$, Michael Springborg ${ }^{2}$

$$
\begin{aligned}
& \text { 1. Institut für Physikalische Chemie, Technische Universität Dresden, D-01062 } \\
& \text { Dresden, Germany } \\
& \text { 2. Institut für Physikalische Chemie, Universität des Saarlandes, D-66123 } \\
& \text { Saarbrücken, Germany }
\end{aligned}
$$

Due to the quantum-confinement effect the optical properties of cadmium sulfide clusters tune with respect to their size. Clusters up to $5 \mathrm{~nm}$ in diameter $(\sim 3750$ atoms), derived from the electronically almost degenerated crystal structures of cadmium sulfide, wurtzite and zinc blende (sphalerite), were studied systematically using a LCAO-DFT-LDA method (DFTB) ${ }^{1,2}$. With a time-dependent extension (linear response) of this method (TD-DFRT-TB) ${ }^{2}$ the optical spectra of bare and surface saturated cadmium sulfide clusters were calculated. Since there is a strong dependency of the lowest unoccupied molecular orbitals on structural properties, basically on the number of single-bonded surface atoms, the values of the $\mathrm{HOMO} / \mathrm{LUMO}$ gap energies trend to zero for clusters having a large number of single bonded Cadmium atoms ${ }^{3}$. It is shown that this effect, caused by low lying cadmium $5 \mathrm{~s}$ states, disappears when surfactants are added to these clusters, namely hydrogen atoms and thiol-groups. The spectra show an asymptotical decreasing decay of the lowest singlet excitation energy toward the value of the bulk band gap of CdS (zinc blende) with increasing size, as found experimentally (e.g. Ref [5]).
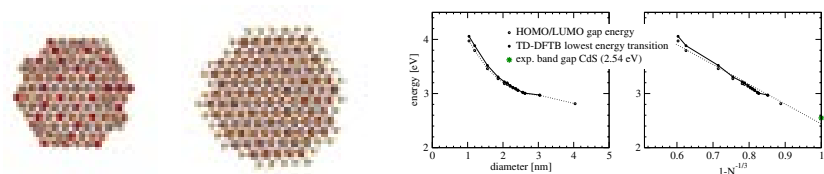

1] Porezag,D. etal, Phys. Rev. B 51, 12947 (1995).

2] Seifert,G. etal, Int. J. Quantum Chem. 58,185 (1996).

[3] Niehaus, T. etal, Phys. Rev. B 63, 085108 (2001).

[4] Joswig, J.-O. etal, J. Phys. Chem B 107, 2897 (2003).

[5] Wang, Y., Herron, N., Phys. Rev. B 42, 7253 (1990).

Methodology and Applications

Hybrid Functionals and their application to small molecules and solids

Joachim Paier, Martijn Marsman, Iann C. Gerber, and Georg Kresse

Institut für Materialphysik and

Center for Computational Materials Science

University of Vienna, Sensengasse 8, A-1090 Wien, Austria

Within the framework of generalized Kohn-Sham schemes, sophisticated techniques, such as exact exchange and hybrid functionals, have made a rather accurate treatment of e.g. small molecules and band gaps in bulk materials possible. A plane-wave based algorithm was implemented in VASP (Vienna Abinitio Simulation Package) to allow for the calculation of exact exchange. Two functionals including exact exchange are presently available in the VASPpackage, namely the PBE0 and the HSE (Heyd-Scuseria-Ernzerhof) functional. A rigorous assessment of the implementation was done by geometry optimization and calculation of the atomization energies of the G2-1 quantum chemical test set, containing 55 molecules of a broad chemical diversity. Excellent agreement compared corresponding Gaussian 03 data and good agreement with experiment was achieved. The mean absolute error (MAE) for atomization energies between VASP energies and the experimental atomization energies is $8.6 \mathrm{kcal} / \mathrm{mol}$ and $3.8 \mathrm{kcal} / \mathrm{mol}$, as calculated with the PBE functional and PBE0 respectively. The mean deviations between VASP and Gaussian are $0.5 \mathrm{kcal} / \mathrm{mol}$ and $0.8 \mathrm{kcal} / \mathrm{mol}$ for the $\mathrm{PBE}$ and PBE0 functional, respectively.

In order to study the properties of bulk materials, lattice constants, and bulk moduli of 20 solids comprising ionic, semiconducting and metallic materials, have been calculated using PBE0 and HSE, respectively. The MAE between plane wave results and experimental data for lattice constants is $0.02 \AA$ and $0.03 \AA$ for the PBE0 and HSE functional, respectively. In general the hybride functional seem to improve on the description of insulating periodic systems in a similar way as on the description of small molecules.

[1] J. Paier, R. Hirschl, M. Marsman, and G. Kresse, J. Chem. Phys, submitted.

[2] I.C. Gerber, János G. Ángyán, J. Paier, M. Marsman, and G. Kresse, to be published. 
A new "von-Weizsäcker-type" approach for non local kinetic energy functionals

David García-Aldea and J. E. Alvarellos

Departamento de Física Fundamental, UNED, Apartado 60141, E-28080 Madrid (Spain).

Some of the non local kinetic energy density functionals (KEDFs) proposed in the literature [1-3] yield the von Weizsäcker functional [4] as a natural term and introduce a non-local term with a Thomas-Fermi structure [5]. The flexibility of the non local term allows to introduce a kernel that makes the functional reproduce some specific physical properties of the homogenous electron system, as the linear response function of the free electron gas, throught the second functional derivative of the KEDFs.

Following the idea of the functionals that can reproduce the linear response function of the free electron gas, in this work we propose a new KEDF which has the natural von Weizsäcker term plus a non local term based on a von Weizsäcker structure instead of on a Thomas-Fermi one. The functional has the advantage of a much more simpler differential equation for the kernel and, defining a reference density for the electronic system, is suitable for using in linear scaling calculations.

We present calculations for some simple systems, from atoms to one dimensional systems.

[1] E. Chacón, J. E. Alvarellos, and P. Tarazona, Phys. Rev. B 32, 7868 (1985); P. García-González, J. E. Alvarellos, and E. Chacón, 53, 9509 (1996) and, 57, 4857 (1998); P. García-González, J. E. Alvarellos, and E. Chacón, Phys. Rev. A 54, 1897 (1996) and, 57, 4192 (1998).

[2] L.-W. Wang and M. P. Teter, Phys. Rev. B 45, 13196 (1992)

[3] Y. A. Wang, N. Govind, and E. A. Carter, Phys. Rev. B 58,13 465 (1998) and 60, 16350 (1999).

[4] C. F.von Weizsäcker Z. Phys. 96, 431 (1935).

[5] L. H. Thomas, Proc. Camb. Phil. Soc. 23, 542 (1927); E. Fermi, Z. Phys. 48, 73 (1928). 
Methodology and Applications

Application of DFT to Clay-Polymer Nanocomposites. Adsorption Within Montmorillonite and First Steps for the Polymerisation Reaction

$\underline{\text { Pascal Boulet }^{1}}$, Peter V. Coveney ${ }^{2}$

MADIREL ${ }^{1}$, CNRS UMR 6121, Université Aix-Marseille 1, Centre de Saint-Jérôme, 13397 Marseille Cedex 20, France

Centre for Computational Science, Department of Chemistry ${ }^{2}$,

University College London, University of London, 20 Gordon Street, London WC1H 0AJ, United Kingdom

For more than three decades there has been increasing interest in the study and formulation of new materials based on combining clays and polymers. Most of these materials are exfoliated, namely, the clay particles are dispersed into a polymer matrix in order to improve various properties such as heat or mechanical resistance. By contrast, studies on non-exfoliated materials, where the clay retains its layered structure, are comparatively rare. Interestingly, these materials are ideal to be investigated by DFT using 3D-periodic models. Our work focuses on the understanding of the polymerisation of small acrylate monomers adsorbed within the montmorillonite clay. It has been suggested ${ }^{1}$ that clays can play a key role as catalysts to promote these reactions. Using fully periodic clay models, at the generalised gradient approximation (GGA) level of theory, we study the adsorption of acrylate molecules onto aluminosilicate sheets and to elucidate the first steps of polymerisation that lead to poly(methyl)methacrylate.

[1] P. V. Coveney, J. L. W. Griffin, M. Watkinson, A. Whiting and E. S. Boek, Mol. Simul., 2002, 28, 295.

Methodology and Applications

\section{Study of the Nucleophilic Trifluoromethylation of Carbonyl Compounds. A DFT investigation of the Structures of Trifluoromethylated Hemiaminal Substrates}

Pascal Boulet $^{1}$, T. Billard ${ }^{2}$, B. R. Langlois ${ }^{2}$, H. Chermette ${ }^{3}$

MADIREL $^{1}$, CNRS UMR 6121, Université Aix-Marseille I, Centre de Saint-Jérôme, 13397 Marseille Cedex 20, France

CERCOF $^{2}$, UMR CNRS 5622, Université Claude Bernard Lyon 1, 43 Bd du 11 novembre 1918, F-69622 Villeurbanne, France

Chimie-Physique Théorique ${ }^{3}$, UMR CNRS 5182, Bâtiment Paul Dirac, Université Claude Bernard Lyon 1, 43 Bd du 11 novembre 1918, F-69622 Villeurbanne, France

New organofluorine compounds, and especially, trifluoromethyl-substituted molecules are of huge interest for various applications, for example, in the field of biochemistry. At present, many methods are available to introduce the $\mathrm{CF}_{3}$ moiety into organic substrates (e.g. carbonyl compounds) and nucleophilic trifluoromethylation is one of those. Stable candidates for the methylation reaction are hemiaminals of trifluoroacetaldehyde $\left(\mathrm{C}_{6} \mathrm{H}_{6}-\mathrm{CH}_{2}-\mathrm{C}_{4} \mathrm{H}_{8} \mathrm{~N}_{2}-\mathrm{CH}\left(\mathrm{CF}_{3} \mathrm{OH}\right)\right)^{1}$. However, both the reaction mechanism and the conformation adopted by the reagent are not completely elucidated. This work focuses on the study of the electronic and geometric structures of the hemiaminal compound, both in the gas phase and in presence of solvent molecules. The effect of the solvent has been modelled using a continuum model. Finally, it has been suggested from experiment that, a dimer of the hemiaminal could be responsible for the $\mathrm{CF}_{3}$ transfer, so that structures for this dimer have also been investigated.

[1] G. Blond, T. Billard and B. Langlois, J. Org. Chem., 2001, 66,4826 .
Methodology and Applications

Density Functional Theory Calculations on the $\mathrm{Li}^{+}, \mathrm{Na}^{+}$and $\mathrm{K}^{+}$-MOR Systems. Interaction Energies and Energy barriers for the Cations Diffusion

Irena Deroche ${ }^{1}$, Pascal Boulet $^{2}$, François Henn ${ }^{1}$

LPMC $^{1}$, CNRS UMR 5617, Université de Montpellier II, Place Eugène Bataillon, 34095 Montpellier Cedex 05, France

MADIREL ${ }^{2}$, CNRS UMR 6121, Université Aix-Marseille I, Centre de Saint-Jérôme, 13397 Marseille Cedex 20, France

The study of the positions of charge-balancing cations within zeolites channels is of major importance to understand both the catalytic behaviour of these aluminosilicates and the adsorption and diffusion of adsorbates. This work deals with a comparative study of the $\mathrm{Li}^{+}, \mathrm{Na}^{+}$and $\mathrm{K}^{+}$cations location within the mordenite zeolite for the $\mathrm{Si} / \mathrm{Al}=48$ ratio. A fully periodic cell of the mordenite is investigated using both the local density approximation and ultrasoft pseudopotentials. The procedure for locating the extrema on the potential energy surface is based on the geometry minimisation technique. We will show that, at the vicinity of the aluminium atom, cations are located at an equal distance to two oxygen atoms and that there are several energetically equivalent positions for the cations. The transition state between these minima corresponds to the cation that interacts with a single oxygen atom. Indeed, the calculated activation barriers are in good agreement with both those measured from TSDC experiments ${ }^{1}$ and those obtained from Monte Carlo simulations ${ }^{2}$ To further investigate the cations location with various $\mathrm{Si} / \mathrm{Al}$ ratio our goal is to use Monte Carlo simulations. For this purpose, the energies for various positions of the cations within the channels have been calculated. Classical potentials for the cations, such as the Buckingham potential, are therefore fitted on DFT calculations.

[1] F. Henn, S. Devautour, L. Maati, J.-C. Giuntini, J. V. Zanchetta, H. Schafer and J. Vanderschueren, Solid State Ionics, 2000, 136-13\%, 1335

[2] G. Maurin, P. Senet, S. Devautour, F. Henn and J.-C. Giuntini, J. Chem. Phys., 2002, 117, 1405.

Methodology and Applications

A kinetic energy density study of the semilocal functionals

David García-Aldea and J. E. Alvarellos

Departamento de Física Fundamental, UNED, Apartado 60141, E-28080 Madrid (Spain).

The success of the Kohn-Sham method [1] for the solution of the $N$ electron problem within the DFT has allowed a wealth of calculations of almost every electronic property of chemical and condensed matter systems. This approach is a $N$-electron problem, in a $3 N$ dimensional space. However, the original Hohenberg-Kohn idea keeps its appeal after the years, because total energy can be directly evaluated in the three dimensional space, where the density is the fundamental variable.

Usually, the semilocal kinetic energy density functionals (KEDF) [2,3], evaluated for the KS densities, yield results for the total kinetic energy within about a $1 \%$ error. Nevertheless, when the same functionals are used in a variational scheme densities with incorrect behaviors are obtained [4]

In this work we present an exhaustive study of the kinetic energy density (KED) [5] of the semilocal KEDFs, discussing for each case the approximated and exact KEDs. We have defined a parameter able to describe the quality of the KED related with the semilocal functionals.

As a conclusion, we have found all semilocal functionals give overall improper KEDs. On the other hand, those semilocal functionals which are based on corrections over the Thomas-Fermi functional do not improve the KEDs obtained for the Thomas-Fermi itself.

[1] W. Kohn and L. J. Sham, Phys. Rev. 140 (1965) A1133.

[2] A. J. Thakkar, Phys. Rev. A 46, 6920 (1992).

[3] D. J. Lacks and R. G. Gordon, J. Chem. Phys. 100, 4446 (1994).

[4] P. García-González, J. E. Alvarellos, and E. Chacón, Phys. Rev. A 54, 1897 (1996)

[5] E. Sim, J. Larkin, K. Burke, C. W. Bock. Journal of Chemical Physics 118, 8140 (2003). 
